\title{
Topological Semimetals Predicted from First-principles Calculations
}

\author{
Hongming Weng, ${ }^{1,2, *}$ Xi Dai, ${ }^{1,2, \dagger}$ and Zhong Fang ${ }^{1,2, \ddagger}$ \\ ${ }^{1}$ Beijing National Laboratory for Condensed Matter Physics, \\ and Institute of Physics, Chinese Academy of Sciences, Beijing 100190, China \\ ${ }^{2}$ Collaborative Innovation Center of Quantum Matter, Beijing, China
}

\begin{abstract}
We have given a summary on our theoretical predictions of three kinds of topological semimetals (TSMs), namely, Dirac semimetal (DSM), Weyl semimetal (WSM) and Node-Line Semimetal (NLSM). TSMs are new states of quantum matters, which are different with topological insulators. They are characterized by the topological stability of Fermi surface, whether it encloses band crossing point, i.e., Dirac cone like energy node, or not. They are distinguished from each other by the degeneracy and momentum space distribution of the nodal points. To realize these intriguing topological quantum states is quite challenging and crucial to both fundamental science and future application. In 2012 and 2013, $\mathrm{Na}_{3} \mathrm{Bi}$ and $\mathrm{Cd}_{3} \mathrm{As}_{2}$ were theoretically predicted to be DSM, respectively. Their experimental verifications in 2014 have ignited the hot and intensive studies on TSMs. The following theoretical prediction of nonmagnetic WSM in TaAs family stimulated a second wave and many experimental works have come out in this year. In 2014, a kind of three dimensional crystal of carbon has been proposed to be NLSM due to negligible spin-orbit coupling and coexistence of time-reversal and inversion symmetry. Though the final experimental confirmation of NLSM is still missing, there have been several theoretical proposals, including $\mathrm{Cu}_{3} \mathrm{PdN}$ from us. In the final part, we have summarized the whole family of TSMs and their relationship.
\end{abstract}




\section{Table of Contents}

1. Introduction

2. Dirac Semimetal

2.1: $\mathrm{Na}_{3} \mathrm{Bi}$

2.2: $\mathrm{Cd}_{3} \mathrm{As}_{2}$

3. Weyl Semimetal

3.1: Magnetic Weyl Semimetal: $\mathrm{HgCr}_{2} \mathrm{Se}_{4}$

3.2: Non-magnetic Weyl Semimetal: TaAs family

4. Node-Line Semimetal

4.1: All Carbon Mackay-Terrones Crystal

4.2: Anti-Perovskite $\mathrm{Cu}_{3} \mathrm{PdN}$

5. Discussion and Prospect

6. Acknowledgement

7. References

\section{Introduction}

Classification of electronic states is one of the key concepts in condensed matter physics. An usual way to classify the electronic states in solids is based on the symmetry principle, by which most of the states as well as the transitions among them can be understood. For example, the ferro-electric and ferro-magnetic states can be understood as states that break the spacial inversion and time reversal symmetries, respectively. The Landau theory for phase transitions is very successful in condensed matter physics, which is built entirely on the symmetry principle. In the recent thirty years, another way to classify condensed matter systems has been developed through a completely different point of view, i.e. topology. The first type of systems which can be classified by its topological features is the $2 \mathrm{D}$ electron gas under high magnetic field. Once there are integer number of Landau levels being fully 
occupied, the system is in $n$-th integer quantum Hall state (IQHS) with quantized Hall conductance. ${ }^{1}$ Further analysis of IQHS by TKNN revealed that the IQHS has very deep topological origin., ${ }^{2,3}$ Although different IQHS are the same in terms of symmetry, they can be characterized by different integers (TKNN number or Chern number), which can be expressed by the integral of the Berry's curvature over the whole magnetic Brillouin zone (BZ). ${ }^{2}$ This integer number is called topological invariance, which can not be changed without closing the energy gap of the system.

After the discovery of IQHS, it was anticipated that the concept of topological electronic states should not be limited only to 2DEG under external magnetic field ${ }^{4}$. The idea ought to be generalized to the realistic materials as well. In about ten years ago, the idea of finding electronic states that can be classified by topological invariance in solid state materials has been realized in a class of band insulators, topological insulator. ${ }^{5-8}$ Similar with the IQHS, the TI can be characterized by topological invariances or indices but the difference is that these indices, called Z2 indices, can only take two possible value, even or odd. The TI, characterized by odd Z2 indices, has unique Dirac like surface (or edge) states, which is unavoidable as long as the time reversal symmetry is preserved. The first TI proposed by theorists is graphene ${ }^{9}$, while due to its extremely small energy gap (about $10^{-3} \mathrm{meV}$ ) opened by spin-orbit coupling, ${ }^{10}$ it hard to observe any physical properties of TI in graphene. The prediction of TI state in $\mathrm{HgTe} / \mathrm{CdTe}$ quantum well was made by the Stanford group ${ }^{11}$ and had been confirmed experimentally a year after. ${ }^{12}$ The discovery of 3D TI $\mathrm{Bi}_{2} \mathrm{Se}_{3}$ family ${ }^{13-15}$ makes it much easier to study all kinds of properties of TI including the transport under magnetic field, thermal electric properties ${ }^{16,17}$ as well as the possible topological superconductor phase induced by proximate effect. ${ }^{18-20}$ With Doping of the magnetic elements into the $\mathrm{Bi}_{2} \mathrm{Te}_{3}$ family thin films, another long awaited topological phase, quantum anomalous Hall state (QAHE) $)^{4,21-23}$, has been theoretically predicted ${ }^{24}$ and experimentally observed. ${ }^{25}$ The QAHE can be viewed as a kind of IQHS without Landau Levels (no external magnetic field). ${ }^{23}$

With the successful discovery of those topological insulating states, it is nature to ask wether or not metals can be classified by topological invariances as well. Although till now the general topological classification for metals is still unclear, we now have ways to classify a special type of metals, the semimetals, where the zero energy contour in energy dispersion or Fermi surface contains only isolated points (or lines for 3D systems) rather 
than surfaces. These Fermi nodes (or nodal lines) are caused by the band crossing points (also called as nodes or nodal points) right at the Fermi level. The semimetal can be viewed as the special type of "insulator" as well, where the energy gap only closes at those isolated $k$-points or lines. In 2D systems, graphene can be viewed as the first well studied topological semimetal (TSM), where the low energy physics can be well described by the 2D massless Dirac equation. The line integral of the Berry connection along any closed loops in the BZ will accumulate a phase $\pi(0)$ if the loop (doesn't) encloses the Dirac point and this phase can be used as the topological invariance, which manifests itself in the unique Landau level structure under magnetic field. While as we will introduce below, similar to graphene the band crossing points can also occur in 3D materials. Although the band crossings in 3D and $2 \mathrm{D}$ do share some similarities, it is indeed very different. The most essential difference comes from the factor that without extra degeneracy, i.e. the spin degeneracy for fermions, the band crossings in 3D can only be shifted but not removed by small perturbations in Hamiltonian. While in comparison, the band crossing points in 2D is caused by crystalline symmetry on some special $k$-points in the BZ and will disappear immediately once a small perturbation is added to break that symmetry. Therefore in 3D, the crossing points between two nondegenerate bands can be viewed as "topological defects", which are the stable objects like the vortices in the fluid. Such crossing points are called Weyl points (Weyl nodes) because the low energy physics around them can be well described by the Weyl equation, which is well known in particle physics and contains only half of the freedom of the Dirac equation. ${ }^{26}$ Weyl nodes in crystalline solids were first studied as magnetic monopoles in momentum space in the context of anomalous Hall effect ${ }^{27}$ As will be illustrated in detail below, Weyl points have chirality defined as the sign of the determinant for the velocity tensor, which is another unique point in 3D. It has been proved mathematically that Weyl points generated in any lattice model can only come in pairs with opposite chirality. ${ }^{28-30}$ In fact, the only way to remove Weyl points is to move a pair of Weyl points with opposite chirality to a same k point and annihilate them. The most important effect generated by these "chiral electrons" is so called "chiral anomaly" describing the adiabatic electron pumping caused by the joint effect of the external magnetic and electric fields between Weyl points with opposite chirality. ${ }^{31}$ The chiral anomaly (or ABJ anomaly) will generate current along the direction of the magnetic field leading to negative magneto resistance when the magnetic and electric fields are parallel to each other. ${ }^{32,33}$ Besides the negative MR, chiral anomaly 
can also generate more effects in transport ${ }^{34}$ and optical properties, ${ }^{35-38}$ as well as the chiral magnetic effect ${ }^{39,40}$, nonlocal transport ${ }^{41}$ and chiral gauge anomaly. ${ }^{42}$

The nodal point behaves like the magnetic monopole in crystal momentum space, being a "source" or "sink" of Berry curvature, the field of Berry flux. ${ }^{23,27,43}$ Fig. 1 shows the typical band structure and the topological relationship between Fermi surface (with Fermi level slightly off the node) and magnetic monopoles. The Dirac semimetal (DSM) has its Fermi surface encloses two monopoles with opposite topological charge "kissing" at the same $k$-point, while WSM always has opposite monopoles in pair and they are well separated. ${ }^{30}$ Node-Line semimetal (NLSM) is a special case with nodal points forming a periodically continuous line or closed ring in the momentum space. After a loop of adiabatic evolution along a closed path interlocked with the nodal ring, eigenstates on one of the crossing bands will acquire a Berry phase of $\pi$, which stabilize its ring like Fermi surface. Therefore, these three kinds of TSM can be identified by the degeneracy and distribution of nodal points.

These exotic nontrivial TSMs are expected to have intriguing quantum phenomena and physical properties. Their realistic material realization become very important and crucial to bring them into the realm of experimental study and potential application in future. As we have pointed out in Ref. 44, the quick development of first-principles calculation methods and software packages, together with the intrinsic robustness of band topology immune to perturbation and numerical error, ensure the predictive power of the stat-of-art first-principles calculation for the topological materials. The remarkable successful stories include the 2D TIs ${ }^{11,12}$, 3D TIs ${ }^{13-15}$, Chern insulators supporting quantum anomalous Hall effect $^{24,25}, 3 \mathrm{D}$ topological crystalline insulators ${ }^{45,46}$ and so on. In this topical review, we will briefly summarize our theoretical material proposals for three types of TSMs, namely DSM, WSM and NLSM. Some of them have been confirmed by experimental observations after our predictions. Experimentally, the most direct and efficient way to observe the TSMs is to use the angle resolved photoemission spectroscopy (ARPES) technique, which can detect the band structure and even its spin polarization. There are basically three important hallmarks of TSMs to be observed by ARPES, including the 3D Dirac/Weyl points, the Fermi arcs on the surface ${ }^{47}$ and the spin texture of Fermi arcs.

\section{Dirac Semimetal}




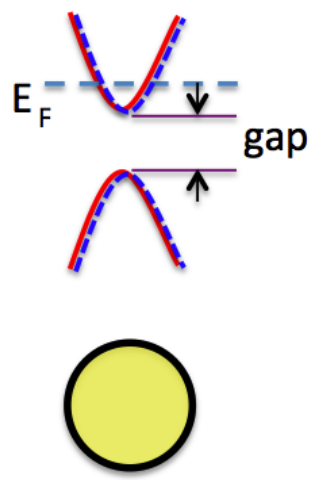

Normal metal
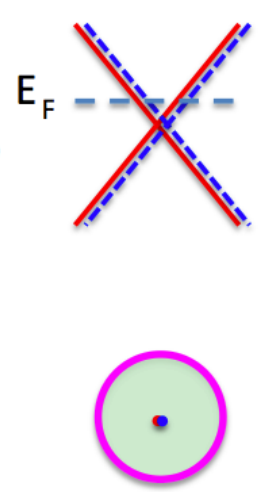

DSM
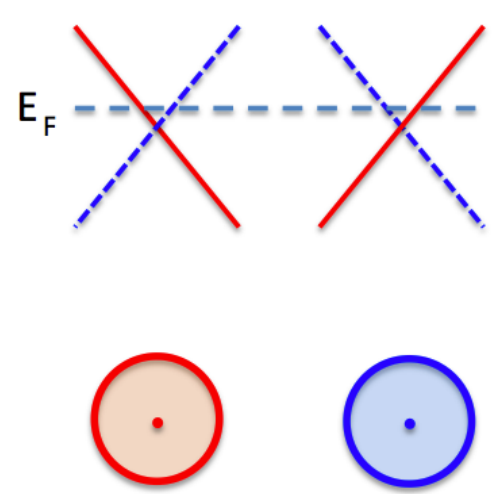

WSM
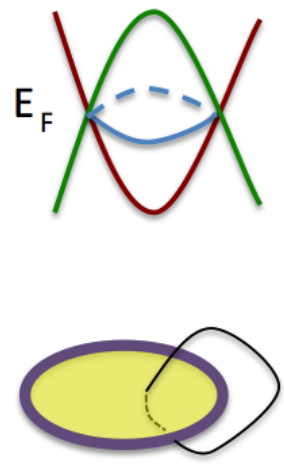

NLSM

FIG. 1: (Color online) Schematic band structure and Fermi surface (with Fermi level shift slightly off the nodal points) for normal metal and three kinds of topological semimetals, Dirac semimetal (DSM), Weyl semimetal (WSM) and Node-Line Semimetal (NLSM). The close path (black thin circle) interlocked with the nodal ring (thick circle) is also shown.

The Dirac equation describing electrons with relativistic effect ${ }^{48}$ is written as

$$
\left(\begin{array}{cc}
\hat{E}(\mathbf{k})-\sigma \cdot \hat{\mathbf{k}} & 0 \\
0 & \hat{E}(\mathbf{k})+\sigma \cdot \hat{\mathbf{k}}
\end{array}\right) \psi=m c^{2}\left(\begin{array}{cc}
0 & I_{2} \\
I_{2} & 0
\end{array}\right) \psi .
$$

Here $m$ represents the mass term and couples the two massless Weyl fermions described by $\pm \sigma \cdot \mathbf{k}$ with opposite chirality. ${ }^{26,49,50}$ If $m$ is finite, this equation describes the massive Dirac fermion as shown in Fig. 1 assuming the Fermi level inside of the gap. If $m$ is zero, it describes the massless Dirac fermion composed by two massless Weyl nodes overlapping each other. If Fermi level is slightly shifted off the Dirac node, the Fermi surface encloses a pair of Weyl nodes. The number of pairs can be looked as a topological invariance to identify DSM.

In a general band inversion mechanism as shown in Fig. 2, the band inversion between two spin degenerate bands can cause four-fold degenerate energy nodal points. However, in general situation as discussed by S. Murakami, ${ }^{51,52}$ including SOC will open a band gap at the four-fold degenerate band crossing points. This leads to insulating state and most probably the system becomes a TI, or a topological crystalline insulator, or any other insulator. However, we think such band gap opening is not inevitable and it can be well protected by some crystal symmetry as long as the two spin degenerate bands belong to two different 2D irreducible representations of the little group at the $k$-point where the band 


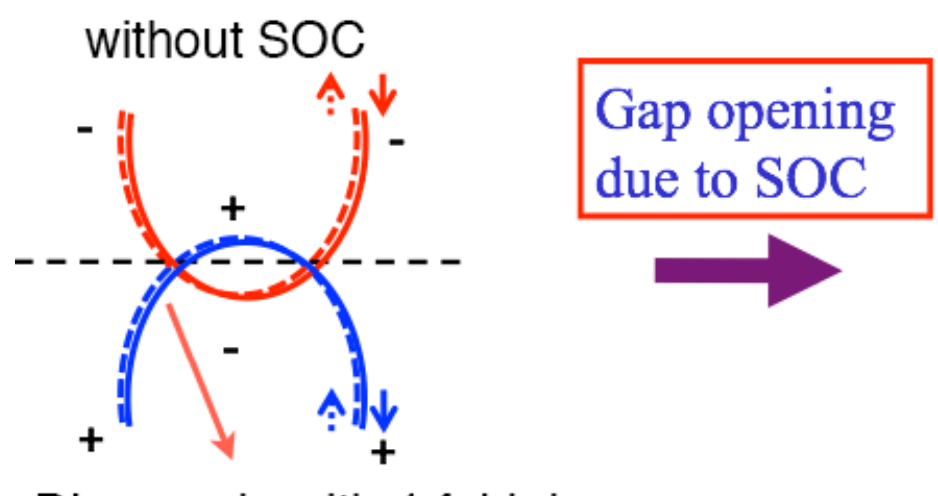

Dirac node with 4-fold degeneracy

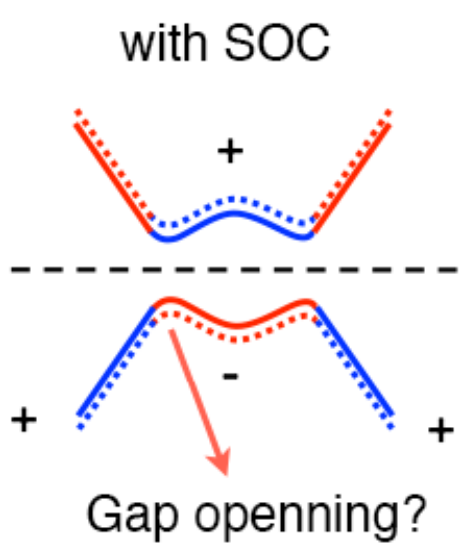

FIG. 2: (Color online) Schematic plot of band inversion mechanism. (left) The case without SOC, band inversion between two spin degenerate (dashed line for up spin, solid one for down spin) bands. + and - indicates the parity of the states at time-reversal invariant moment. (right) After including SOC, the band crossings are lifted and band gap is open.

crossing happens. Therefore, the candidate of DSM must have at least two different 2D irreducible representations in its double space group. This is a necessary condition but not enough one. This finding is very crucial and it directly guides the theoretical prediction of DSM in $\mathrm{Na}_{3} \mathrm{Bi}^{53}$ and $\mathrm{Cd}_{3} \mathrm{As}_{2} .{ }^{54}$

\section{1: $\mathrm{Na}_{3} \mathrm{Bi}$}

In 2012, present authors and their collaborators predicted that DSM might be realized in some hexagonal phase of alkali pnictides $A_{3} B(A=$ Alkali metal, $B=\mathrm{As}$, Sb or Bi) represented by $\mathrm{Na}_{3} \mathrm{Bi}^{53}$ Its crystal structure is shown in Fig. 3. There are two nonequivalent $\mathrm{Na}$ sites, $\mathrm{Na}(1)$ and $\mathrm{Na}(2)$, in one unit cell. $\mathrm{Na}(1)$ and $\mathrm{Bi}$ form simple honeycomb lattice layers stacking along the $c$-axis. $\mathrm{Na}(2)$ atoms are inserted at the interlayer position, connecting these layers on top of Bi atoms. From the ionic picture, due to the closed-shell configuration where the number of valence electrons $\left(3 \times \mathrm{Na}-s^{1}+\mathrm{Bi}-p^{3}\right)$ is equal to 6 , one may expect $\mathrm{Na}_{3} \mathrm{Bi}$ is a semiconductor, similar to $\mathrm{Na}_{3} \mathrm{Sb}^{55}$. However, the electronegativity of $\mathrm{Bi}$ is weaker than $\mathrm{Sb}$, which makes $\mathrm{Na}_{3} \mathrm{Bi}$ be DSM rather semiconductor like $\mathrm{Na}_{3} \mathrm{Sb}$.

The calculated band structures of $\mathrm{Na}_{3} \mathrm{Bi}$ are shown in Fig. 4. They suggest that the valence and conduction bands are dominated by Bi-6p and Na-3s states. Around the Fermi level, the top valence band is mostly from $\mathrm{Bi}-6 p_{x, y}$ states and the lowest conduction band is 


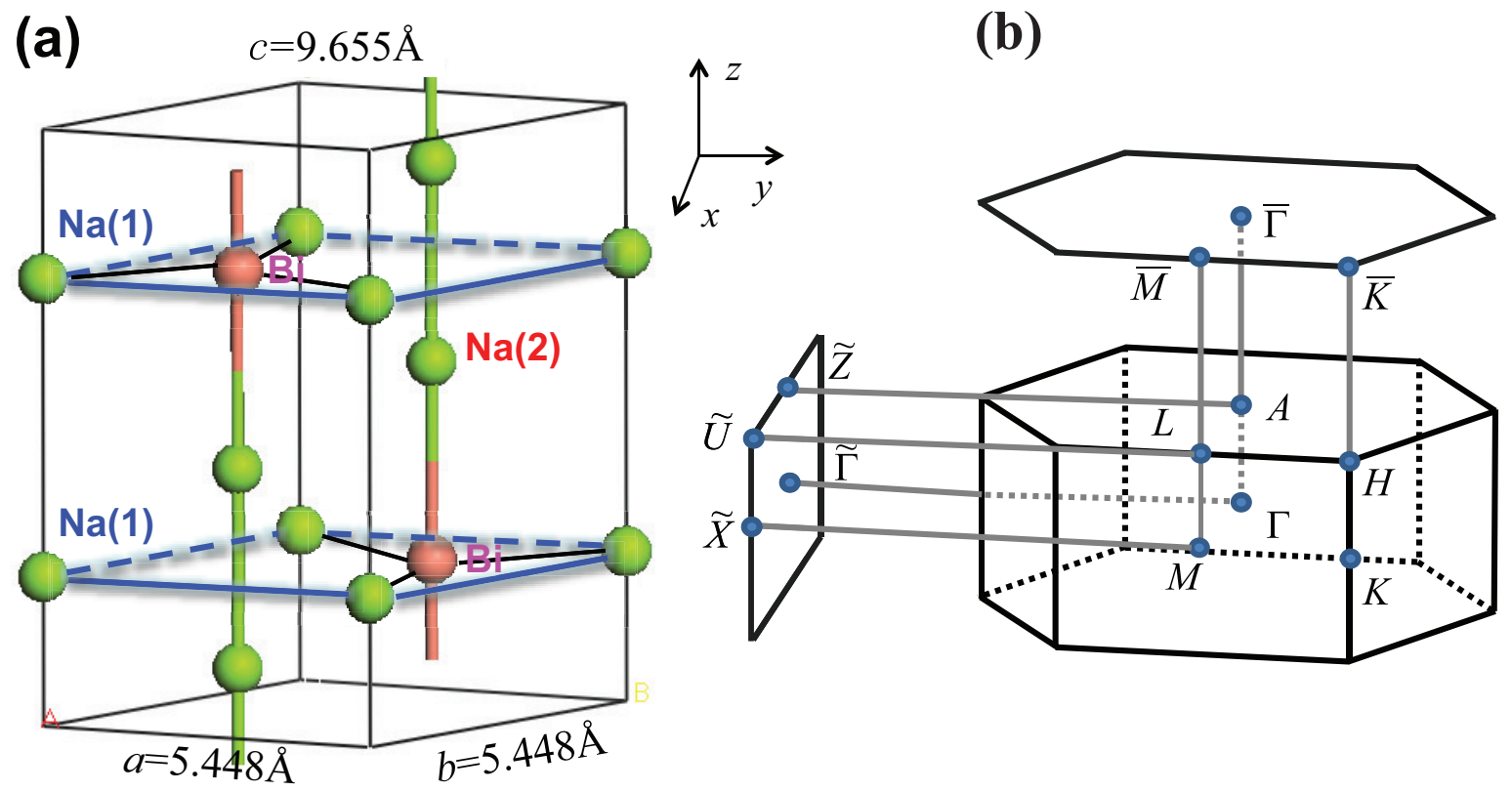

FIG. 3: (Color online) (a) Crystal structure of $\mathrm{Na}_{3} \mathrm{Bi}$ with $P 6_{3} / m m c$ symmetry. $\mathrm{Na}(1)$ is at $2 b$ position $\pm\left(0,0, \frac{1}{4}\right)$, and $\mathrm{Bi}$ is at $2 c$ position $\pm\left(\frac{1}{3}, \frac{2}{3}, \frac{1}{4}\right)$. They form honeycomb lattice layers. $\mathrm{Na}(2)$ are at $4 f$ position $\pm\left(\frac{1}{3}, \frac{2}{3}, u\right)$ and $\pm\left(\frac{2}{3}, \frac{1}{3}, \frac{1}{2}+u\right)$ with $u=0.583$, threading Bi along $c$ axis. (b) Brillouin Zone of bulk and the projected surface Brillouin Zones of (001) and (010) plane.

mostly from $\mathrm{Na}(1)-3 s$ orbital. These features are similar to those of $\mathrm{Na}_{3} \mathrm{Sb}^{55}$ except that in $\mathrm{Na}_{3} \mathrm{Bi}$, the Na-3s band is lower than $\mathrm{Bi}-6 p_{x, y}$ by about $0.3 \mathrm{eV}$ at $\Gamma$, and this value is enhanced to be $0.7 \mathrm{eV}$ in the presence of SOC. This is a typical picture of band inversion ${ }^{44}$ but absent in $\mathrm{Na}_{3} \mathrm{Sb}$. The band inversion is due to the heavier $\mathrm{Bi}$, which has higher $6 p$-states and larger SOC compared to Sb. Since GGA usually underestimates the band gap and results in the overestimation of band inversion, the band inversion is further confirmed by the calculation using hybrid functional HSE ${ }^{56,57}$. The band inversion persisted and Na-3s band is around $0.5 \mathrm{eV}$ lower than Bi- $6 p$ ones. $\mathrm{K}_{3} \mathrm{Bi}$ and $\mathrm{Rb}_{3} \mathrm{Bi}$ have the similar band structure and band topology with band inversion around $0.33 \mathrm{eV}$ and $0.42 \mathrm{eV}$, respectively.

Different from topological insulators such as $\mathrm{Bi}_{2} \mathrm{Te}_{3}$ and $\mathrm{Bi}_{2} \mathrm{Se}_{3},{ }^{13,58} \mathrm{Na}_{3} \mathrm{Bi}$ is a semimetal with two nodes (band-crossings) exactly at Fermi level as shown in Fig. 4. Its Fermi surface consists of two isolated Fermi points, which are located at $\left(0,0, k_{z}^{c} \approx \pm 0.26 \times \frac{\pi}{c}\right)$ along the $\Gamma$-A line. Around each node, the band dispersions is linear, resulting in a 3D Dirac cone. It is different from the Dirac cone in graphene not only in dimensionality, but also in its 

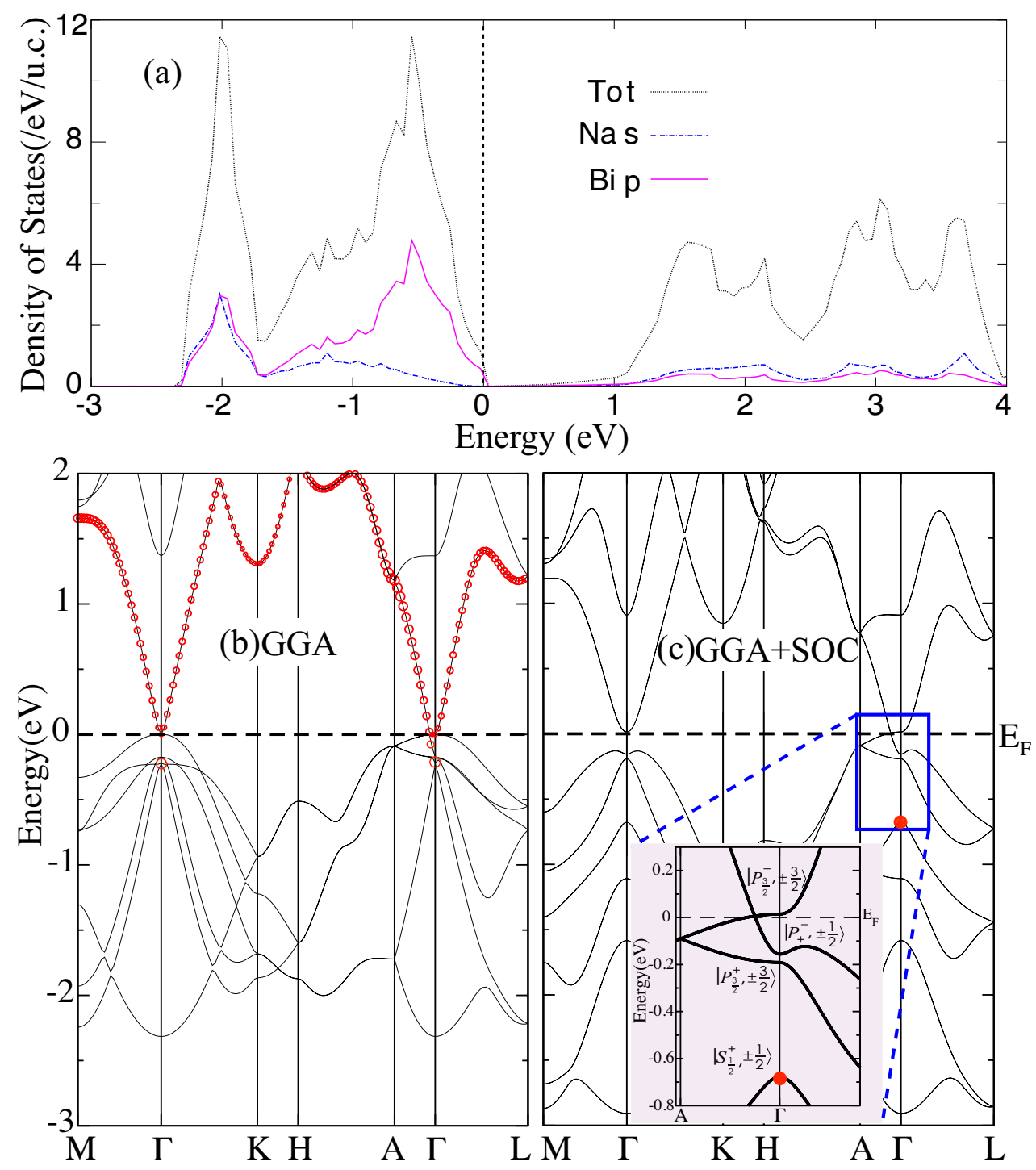

FIG. 4: (Color online) The calculated electronic structures of $\mathrm{Na}_{3} \mathrm{Bi}$. (a) The total and partial density of states. (b) and (c) are the band structures without and with spin-orbit coupling, respectively. The red circles indicate the projection to the Na-3s states. The orbital characters of wave-functions at $\Gamma$ point are labeled in the inset (see Effective Hamiltonian for details).

robustness, because the Fermi points here survive in the presence of SOC, but a tiny band gap open in graphene. ${ }^{10}$

The crossing bands along the $\Gamma$-A line belong to two different irreducible representations distinguished by the three-fold rotational symmetry around $\Gamma$-A axis. Breaking this symmetry will introduce interaction between them and make it insulating. It has been tested that 

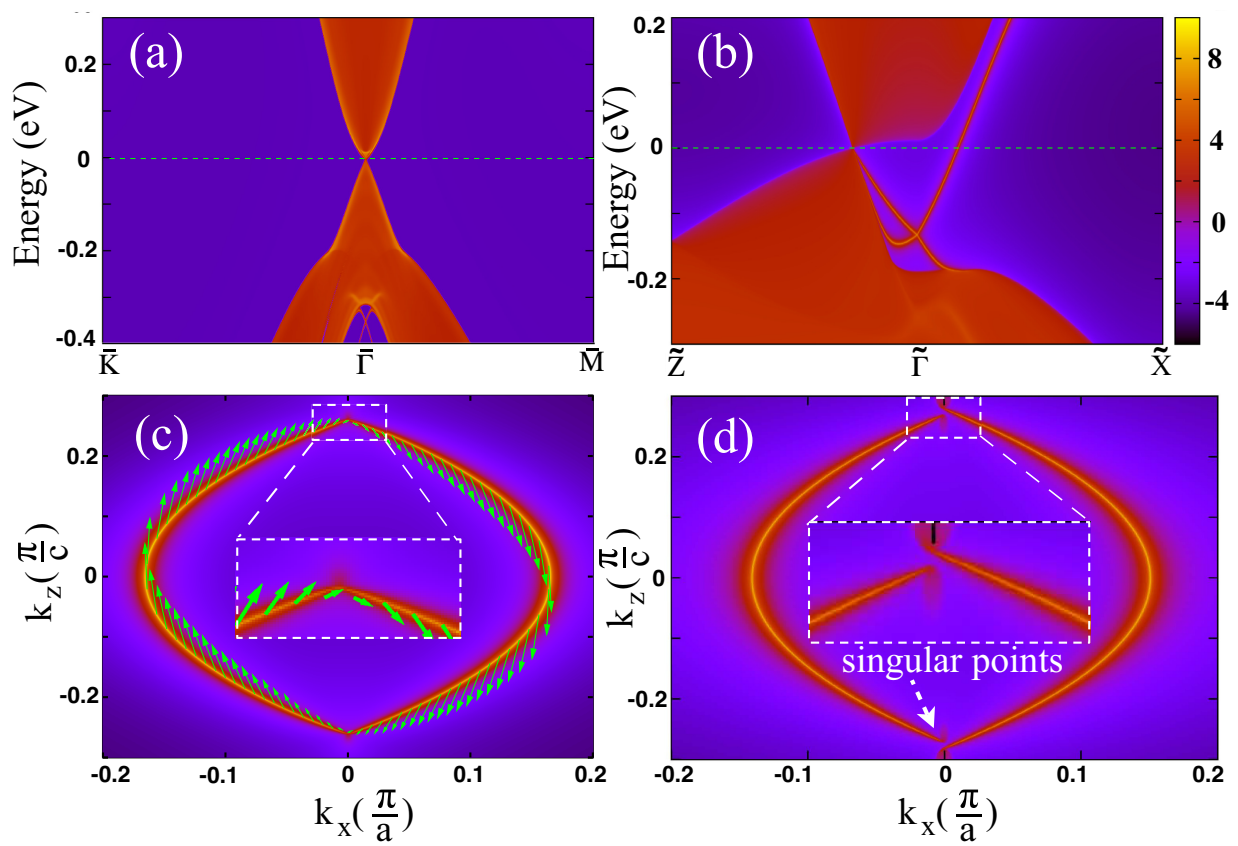

FIG. 5: (Color online) The projected surface states and their fermi surfaces of $\mathrm{Na}_{3} \mathrm{Bi}$. (a) and (b) are the projected surface density of states for [001] and [010] surfaces, respectively. (c) The fermi surfaces (fermi arcs) and its spin texture (in-plane component) for the [010] surface states. (d) The fermi arcs of [010] surface obtained from fitted effective hamiltonian with additional exchange field $h_{1}=6 \mathrm{meV}$ (see Phase diagram for details). The discontinuity around the singular fermi points becomes now obvious (enlarged in the insets).

$1 \%$ compression along $y$ axis will open up a gap $\approx 5.6 \mathrm{meV}$. This insulating state, however, is topologically non-trivial with $Z_{2}=1^{7,8}$ due to the inverted band structure around the $\Gamma$ point. This fact leads to coexistence of both bulk 3D Dirac cone and topological surface states (a single pair) as long as the crystal symmetry protecting band crossings stands. As shown in Fig. 5(a), the solid Dirac cone is from the projection of bulk 3D Dirac cone onto [001]-surface overlapped with the topological surface states. Such distinguished property of DSM is more obvious if inspecting its side surface on which two Dirac cones are projected at two different points. As shown in Fig. 5(b), the solid Dirac cone from bulk states is well separated from the topological surface Dirac cone. While the surface states merge into the bulk states at the Dirac points where bulk band gap closes. Thus, if Fermi level passes through the bulk Dirac nodes, the surface Fermi surface has Fermi arc structures as shown in Fig. 5(c). 
It seems that the entire fermi surface is closed, while its derivative and Fermi velocity are ill defined at the two singular points (corresponding to the projection of bulk Dirac points to the surface). The spin texture of surface states has helical structure, quite similar to that of topological insulators, but the magnitude of spin vector vanishes at the singular points. This kind of Fermi surfaces has never been found before, and it can be understood following the discussions for Weyl semimetal. ${ }^{47,59}$ If the $4 \times 4$ Dirac point is split into two separated $2 \times 2$ Weyl points in momentum space by breaking time reversal or inversion symmetry ${ }^{60,61}$, the Fermi surface of surface states will also split into open segments which are Fermi arcs discussed in Weyl semimetal. In Fig. 5(d), an exchange field breaking time-reversal symmetry separates the touch of two Fermi arcs.

All these characters in contrast to conventional metals and topological insulators have been be experimentally confirmed by successive ARPES measurements. ${ }^{62-64}$ The extraordinary magneto transport property in DSM $\mathrm{Na}_{3} \mathrm{Bi}$ due to chiral anomaly has also been confirmed experimentally. ${ }^{65-67}$

\section{2: $\mathrm{Cd}_{3} \mathrm{As}_{2}$}

After the theoretical prediction of DMS in $\mathrm{Na}_{3} \mathrm{Bi}$, the experimental works to confirm it have been intensively performed. It is soon found to be unstable in air. Therefore, the present authors and their collaborators were motivated to find other better materials to be more suitable for experimental studies. The well known compound $\mathrm{Cd}_{3} \mathrm{As}_{2}$ was reinvestigated and was found to be another DSM candidate in $2013 .{ }^{54}$ Similarly, it has a single pair of Dirac points in bulk and Fermi arcs on proper surface. In particular, in one of its phase the spin degeneracy of crossing bands is lifted away from the Dirac points, in star contrast to other examples ${ }^{53,68}$. It is further suggested that its quantum well structure can naturally support the quantum spin Hall (QSH) effect. The other nice aspect of $\mathrm{Cd}_{3} \mathrm{As}_{2}$ is its high carrier mobility up to $15000 \mathrm{~cm}^{2} \mathrm{~V}^{-1} \mathrm{~s}^{-1}$ at room temperature and $80000 \mathrm{~cm}^{2} \mathrm{~V}^{-1} \mathrm{~s}^{-1}$ at $4 \mathrm{~K}$, reported about 50 years ago ${ }^{69}$. These advantages make it a promising candidate for future experimental studies and potential applications.

The crystal structure of $\mathrm{Cd}_{3} \mathrm{As}_{2}$ is complicated. In Fig. 6, there are hypothetical antifluorite $\mathrm{Cd}_{8} \mathrm{As}_{4}$ and zinc blende $\mathrm{Cd}_{4} \mathrm{As}_{4}$. The $\mathrm{Cd}_{6} \mathrm{As}_{4}$ is obtained with two diagonal sites of $\mathrm{Cd}$ vacancy in $\mathrm{Cd}_{8} \mathrm{As}_{4}$. This is the basic building block of real $\mathrm{Cd}_{3} \mathrm{As}_{2}$ crystal structure. If the arrangement of building block is random, i.e., the distribution of these vacancies is random, one may treat it by virtual crystal approximation (VCA) for simplicity ${ }^{70,71}$. However, 


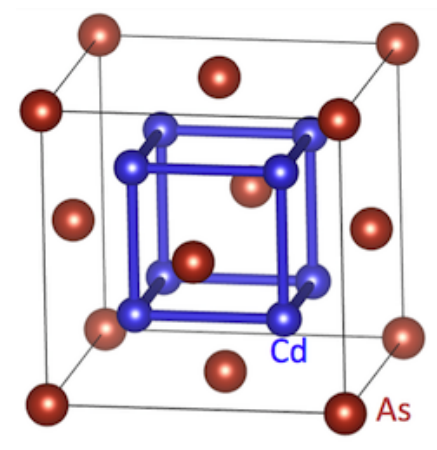

anti- $\mathrm{CaF}_{2}$ $\mathrm{Cd}_{8} \mathrm{As}_{4}$

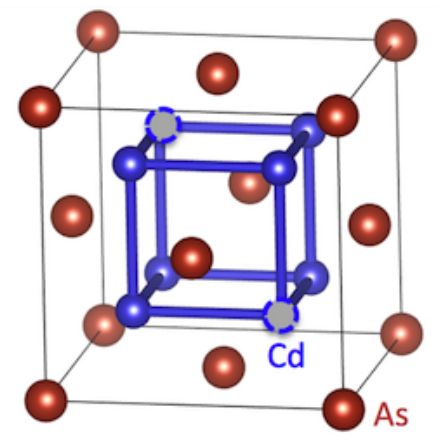

$\mathrm{Cd}_{6} \mathrm{As}_{4}$

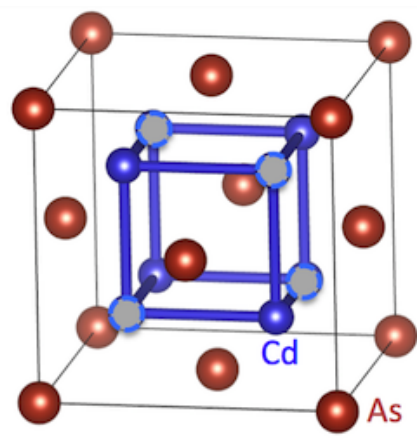

zinc blende $\mathrm{Cd}_{4} \mathrm{As}_{4}$

FIG. 6: (Color online) Crystal structure of $\mathrm{Cd}_{3} \mathrm{As}_{2}$. (left) The hypothetical aniti-CaF 2 structure of $\mathrm{Cd}_{8} \mathrm{As}_{4}$. (middle) Two diagonal sites with $\mathrm{Cd}$ vacancy with $\mathrm{Cd}_{6} \mathrm{As}_{4}$ formula as the building block of real $\mathrm{Cd}_{3} \mathrm{As}_{2}$ crystal structure. (right) The hypothetical zinc blende structure of $\mathrm{Cd}_{4} \mathrm{As}_{4}$.

those vacancies are in fact ordered even at room temperature, leading to a tetragonal structure with $D_{4 h}^{15}\left(\mathrm{P}_{2} / \mathrm{nmc}\right)$ symmetry with 40 atoms per unit cell being $\sqrt{2} \times 2 \times 2$ supercell of building block. (called Structure I hereafter ), or a body centered tetragonal structure with $C_{4 v}^{12}\left(I 4_{1} c d\right)$ symmetry with 80 atoms per unit cell being $2 \times 2 \times 4$ supercell of building block (called Structure II hereafter). The later one is more favored ${ }^{72}$ and we noticed there is correction ${ }^{73}$ to the crystal structure after our original work published. This vacancy ordering and very large uint cell of $\mathrm{Cd}_{3} \mathrm{As}_{2}$ has brings serious problems for theoretical studies in history, and there has been no first-principles calculation before Ref. 54 .

$\mathrm{Cd}_{3} \mathrm{As}_{2}$ belongs to the $\mathrm{II}_{3}-\mathrm{V}_{2}$-types narrow gap semiconductor family. ${ }^{69}$ It has drawn a lot attention since it has been believed to have inverted band structure, ${ }^{74-77}$ whereas all others $\mathrm{Cd}_{3} \mathrm{P}_{2}, \mathrm{Zn}_{3} \mathrm{As}_{2}$ and $\mathrm{Zn}_{3} \mathrm{P}_{2}$ have normal band ordering. In contrast to other inverted band compounds (like $\mathrm{HgTe}, \mathrm{HgSe}$, and $\alpha$-Sn), $\mathrm{Cd}_{3} \mathrm{As}_{2}$ belongs to tetragonal symmetry, and is the representative of this group, which has the splitted valence band top at $\vec{k}=0$.

Similar to most of the semiconductors with anti-fluorite or zinc-blende structures, the low energy electronic properties of $\mathrm{Cd}_{3} \mathrm{As}_{2}$ are mostly determined by the $\mathrm{Cd}-5 s$ states (conduction bands) and the As- $4 p$ states (valence bands), as shown in Fig. 7. However, there are two distinct features: (1) band-inversion around $\Gamma$ with the $s$-band (red solid cycle) lower than the $p$ ones, which is an important sign of non-trivial topology; (2) it is semimetallic with band crossings along the $\Gamma-\mathrm{Z}$ path. This band-crossing is unavoidable since the two 


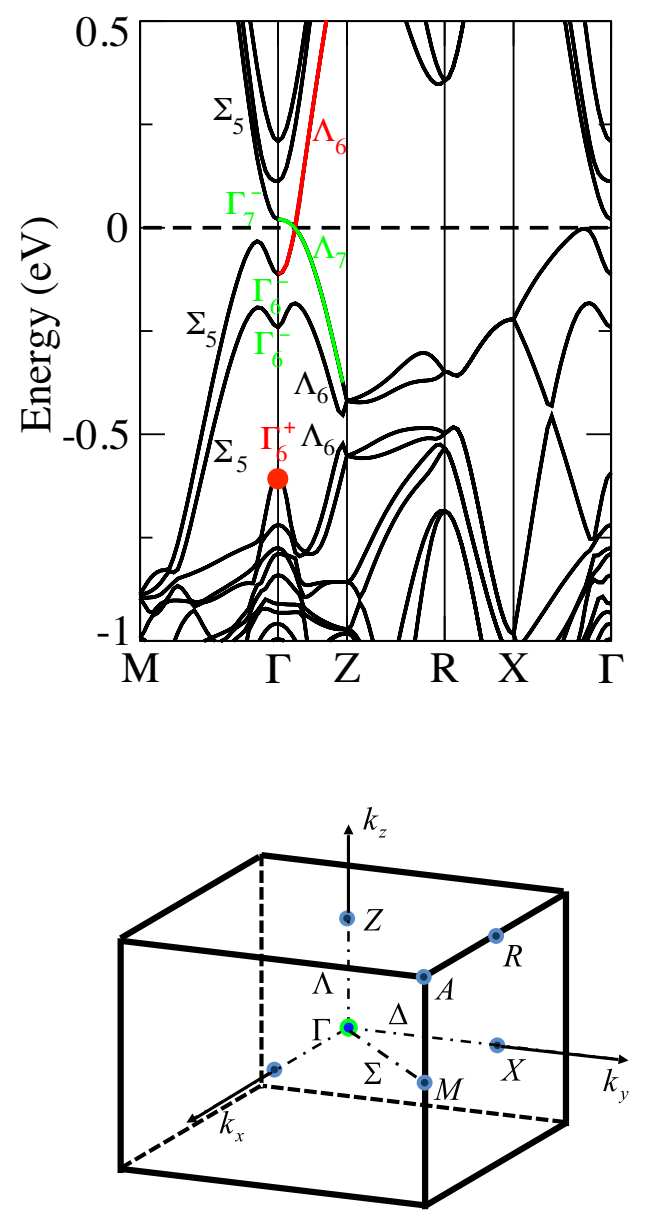

(a) structure I
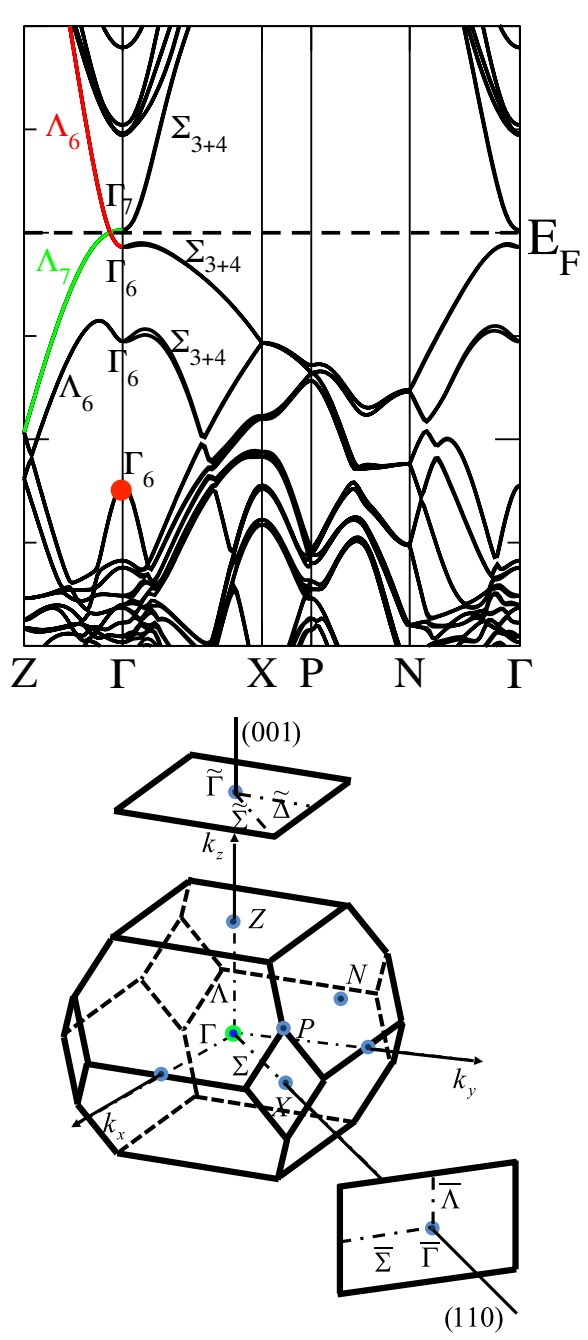

(b) structure II

FIG. 7: (Color online) The calculated band structures for $\mathrm{Cd}_{3} \mathrm{As}_{2}$ with crystal structure I (a) and II (b), as well as the definition of high symmetric points in each Brillouin zone (BZ). The projected surface BZ for structure II is also shown. The representation of selected bands at $\Gamma$ and along high symmetric $\vec{k}$ path are indicated. Solid dots in band structures indicate the projected $s$ bands.

bands belong to different $\left(\Lambda_{6}\right.$ and $\left.\Lambda_{7}\right)$ representations respectively, as distinguished by $C_{4}$ rotational symmetry around $k_{z}$ axis. The different representation prohibits hybridization between them, resulting in the protected band-crossing. The crossing points locate exactly at the Fermi level due to charge neutrality requirement and the Fermi surface has only a single pair of Dirac points (two symmetric points along $\Gamma-Z$ related by TR). Structure I and II share these common features with small but remarkably difference as will be addressed in 
the following.

As had been attempted with perturbation method ${ }^{78}$, vacancy ordering and BZ folding play important role for the band-inversion, in contrast to the cases of $\mathrm{HgTe}$ or $\mathrm{Ag}_{2} \mathrm{Te}^{79}$, where it was driven by the the shallow $d$ states. To prove this, $\mathrm{Cd}_{3} \mathrm{As}_{2}$ with hypothetic anti-fluorite structure without vacancy in Fig. 6(a) has been calculated by using VCA method, compared with the usual first-principles calculation for $\mathrm{Cd}_{3} \mathrm{As}_{2}$ in the same anti-flurite structure but with $\mathrm{Cd}$ vacancy in Fig. 6(b). It is found that the VCA calculation gives out normal band ordering at $\Gamma$ but the one with $\mathrm{Cd}$ vacancy results in inverted band ordering. At the BZ boundary $\mathrm{X}$ point of the hypothetic anti-fluorite structure without $\mathrm{Cd}$ vacancy, there exists shallow $s$ and $p$ states, which will be folded to $\Gamma$ in realistic structure with $\mathrm{Cd}$ vacancy ordering. Therefore, the hybridization among the states with the same representation will push them away from each other, i.e, make the lowest $s$-state even lower and highest $p$-state higher, resulting in the robust band inversion at $\Gamma$. The band-inversion calculated from the generalized gradient approximation (GGA) is about $0.7 \mathrm{eV}$ for both structure I and II. The possible underestimation of the $s-p$ gap within GGA is improved by the calculations with HSE method. ${ }^{56,57}$ The band-inversion around $0.3 \mathrm{eV}$ is obtained, being consistent with most of the existing experimental evidence, such as the optical and transport measurements. ${ }^{74,75}$

The inverted band structure discussed above suggests that $\mathrm{Cd}_{3} \mathrm{As}_{2}$ is topologically nontrivial. However, due to the four-fold rotational symmetry, it is in fact a 3D DSM with a pair of 3D Dirac points at the Fermi level. This is slight different from $\mathrm{Na}_{3} \mathrm{Bi}$. Similarly, if the four-fold rotational symmetry is broken, two Weyl fermions with opposite chirality will be annihilate each other, resulting in a massive Dirac fermions with finite band gap. The resulting insulator is a strong TI.

Similar as $\mathrm{Na}_{3} \mathrm{Bi}, \mathrm{Cd}_{3} \mathrm{As}_{2}$ has coexistence of bulk and surface Dirac cone in its surface state. The semi-infinite (001) and (110) surfaces of the structure II $\mathrm{Cd}_{3} \mathrm{As}_{2}$ are presented in Fig. 8. For (001) surface, the surface projection of continuous bulk states superposes the non-trivial surface states. The Fermi surface of this surface is just a point as shown in Fig. 8(b). For (110) surface, the non-trivial surface states are clear. The Fermi surface of such topological surface states has two half-circle Fermi arcs touching at the singularity points where the surface projection of bulk Dirac points exists.

It is noted that structure II has no inversion symmetry and no Kramer degeneracy at general momenta. As shown in Fig. 9, the bulk Dirac cone in structure II has spin splitting 

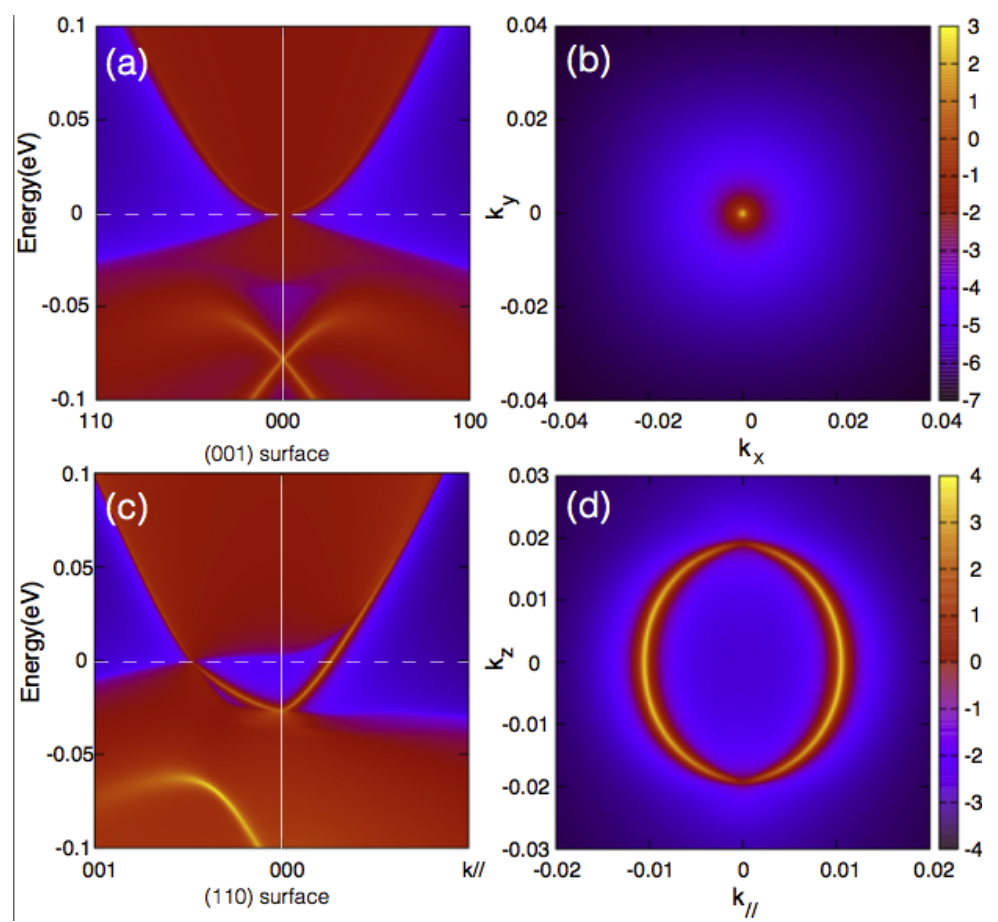

FIG. 8: (Color online) The calculated surface states (left panels) and corresponding fermi surface (right panels) of structure II $\mathrm{Cd}_{3} \mathrm{As}_{2}$ for its (001) (upper panels) and (110) (lower panels) surface.

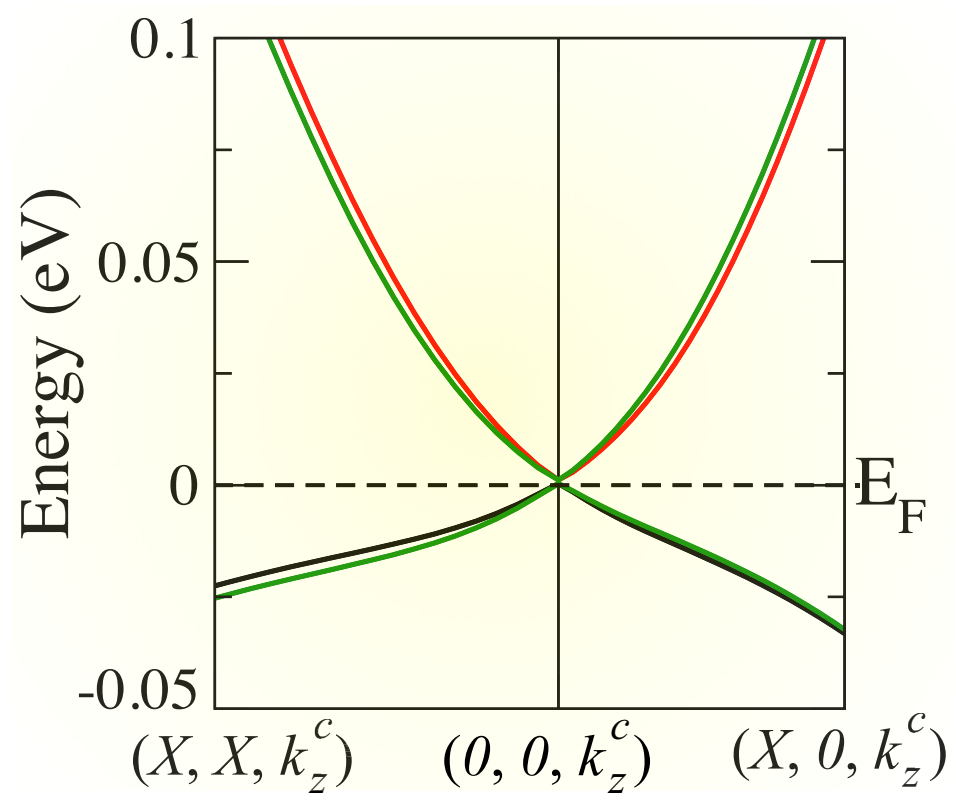

FIG. 9: (Color online) Band dispersions and band-splitting in the plane passing through Dirac point $\left(0,0, k_{z}^{c}\right)$ and perpendicular to $\Gamma$-Z for structure II. $X$ and $k_{z}^{c}$ are around 0.1 and $0.032 \AA^{-1}$, respectively. 
as momentum deviate from the rotation axis due to the inversion symmetry broken. Such spin polarized bulk Dirac cone is quite similar to the Weyl cone in WSM of TaAs family.

Soon after this theoretical prediction, the present authors and their collaborators have observed the 3D bulk Dirac cone ${ }^{80,81}$ and surface states ${ }^{82}$ in $\mathrm{Cd}_{3} \mathrm{As}_{3}$ through ARPES measurement. Other experimental works include Ref. 83-87.

B. Yang and N. Nagaosa have classified $\mathrm{Na}_{3} \mathrm{Bi}$ and $\mathrm{Cd}_{3} \mathrm{As}_{2}$ as DSM carrying quantized topological invariant, ${ }^{88}$ while $\mathrm{BiO}_{2}{ }^{68}$ and distorted spinel ${ }^{89}$ as the class having only a single Dirac point at a time-reversal invariant momentum. The classification of DSM is further investigated by Gibson et al. ${ }^{90}$ and several similar DSM candidates have also been proposed by Du et $a l .{ }^{91}$ As originally pointed out by S. Murakami, ${ }^{51,52}$, DSM can also be obtained at

the topological phase transition point for IS conserved system. $\mathrm{Bi}_{1-x} \mathrm{Sb}_{x}{ }^{92,93}$ is believed to be DSM at the phase transition point from TI to normal insulator. We have proposed that $\mathrm{ZrTe}_{5}$ and $\mathrm{HfTe}_{5}$ are DSM only at the phase transition point from weak to strong TI. ${ }^{40,94-96}$

\section{Weyl Semimetal}

As discussed before, DSM has Dirac nodes composed by two opposite chiral Weyl nodes "kiss" at the same $k$-point due to the degeneracy in spin degree of freedom. In condensed matters, such spin degeneracy at general $k$-point is usually protected by coexistence of timereversal symmetry (TRS) and inversion (IS). It is known as Kramer degeneracy. Breaking either TRS or IS might result in two-fold degenerate Weyl nodes. This leads to two types of WSM, i.e., the magnetic WSM and nonmagnetic noncentrosymmetric WSM. The first WSM was proposed to be in pyrochlore iridates with all-in/all-out magnetic ordering by X. Wan et $a l .{ }^{47}$ In the same year, our collaborators and $\mathrm{us}^{59}$ proposed that ferromagnetic half-metal $\mathrm{HgCr}_{2} \mathrm{Se}_{4}$ might be a WSM with pair of Weyl nodes having topological charge of 2, so called double WSM. ${ }^{97}$ However, the magnetic WSM suffers from the complex domain structure in ARPES measurement. There is still no material definitely confirmed to be magnetic WSM up to now. Finding a nonmagnetic WSM is thus very important. In this section, we will introduce our theoretical predictions of $\mathrm{FM} \mathrm{HgCr}_{2} \mathrm{Se}_{4}$ and noncentrosymmetric nonmagnetic TaAs family. The recent progress in nonmagnetic WSM is the proposal of type-II WSM ${ }^{98}$ in $\mathrm{WTe}_{2} \cdot{ }^{99}$

\section{1: Magnetic Weyl Semimetal: $\mathrm{HgCr}_{2} \mathrm{Se}_{4}$}

In 2011, it was proposed that a spinel structural compound $\mathrm{HgCr}_{2} \mathrm{Se}_{4}$ might be a WSM under its ground state. Compared with the former proposal of $\mathrm{WSM}^{47}, \mathrm{HgCr}_{2} \mathrm{Se}_{4}$ has only 
one pair of Weyl cones around Fermi level and each Weyl cone has topological charge of 2. $\mathrm{HgCr}_{2} \mathrm{Se}_{4}$ has quite simple ferromagnetic (FM) ordering and robust against choice of on-site Coulmb interaction parameter $U$. Before WSM state was proposed in it, $\mathrm{HgCr}_{2} \mathrm{Se}_{4}$ was known as a FM spinel exhibiting large coupling effects between electronic and magnetic properties ${ }^{100}$. It shows quite interesting properties like giant magnetoresistance ${ }^{101}$, anomalous Hall effect ${ }^{102}$, and red shift of optical absorption edge ${ }^{103}$. Its experimental Curie temperature $T_{c}$ is high (around $106 \sim 120 \mathrm{~K}$ ), and the saturated moment is around 5.64 $\mu_{B} /$ f.u. ${ }^{104,105}$, approaching the atomic value expected for high-spin $\mathrm{Cr}^{3+}$. Its transport behavior is different from other FM chalcogenide spinels, like $\mathrm{CdCr}_{2} \mathrm{Se}_{4}$ and $\mathrm{CdCr}_{2} \mathrm{~S}_{4}$ which are clearly semiconducting. $\mathrm{HgCr}_{2} \mathrm{Se}_{4}$ exhibits semiconducting character in the paramagnetic state but metallic in the low temperature FM phase ${ }^{101,106,107 .}$

The spinel structure, with space group Fd⿳亠丷⿵冂丶 diamond structures. As shown in Fig. 10, taking $\mathrm{Cr}_{2} \mathrm{Se}_{4}$ cluster as a single pseudo-atom (called X) located at the center of mass, $\mathrm{HgX}$ becomes a typical zinc blende structure. The pseudo atom $\mathrm{X}$ is actually a small cube formed by $4 \mathrm{Cr}$ atoms and 4 Se atoms located at the cube corners alternatively. The cubes are connected by sharing the $\mathrm{Cr}$ atoms at the corner. As the result, each $\mathrm{Cr}$ atom is octahedrally coordinated by 6 nearest Se atoms. There are two $\mathrm{HgX}$ formula unit (f.u.) in each unit cell, and they are connected by the inversion symmetry similar to the two atoms in the diamond structure.

The first-principles calculation confirms that FM solution is considerably more stable than non-magnetic solution by about $2.8 \mathrm{eV} / \mathrm{f}$.u. lower in totoal energy. The obtained magnetic moment $\left(6.0 \mu_{B}\right.$ /f.u.) agrees with experimental value ${ }^{104,105}$ very well. The electronic structures shown in Fig. 11 (a) and (b) suggest that it can be approximately characterized as a "zero-gap half-metal" in the case without spin-orbit coupling (SOC). It is zero-gap because there is band touching around $\Gamma$ at Fermi level in spin down channel; it is half-metal since there band gap in the up-spin channel. The $3 d$-states of $\mathrm{Cr}^{3+}$ are in the configuration of $t_{2 g}^{3 \uparrow} e_{g}^{0 \uparrow} t_{2 g}^{0 \downarrow} e_{g}^{0 \downarrow}$. The band gap is due to crystal field splitting of the $t_{2 g}^{3 \uparrow}$ and $e_{g}^{0 \uparrow}$ manifolds. The Se- $4 p$ states are from -6 to $0 \mathrm{eV}$, being fully occupied and contribute to the valence band maxima dominantly. By hybridizing with Cr-3d states, Se- $4 p$ are slightly spin-polarized but with opposite moment (about $-0.08 \mu_{B} / \mathrm{Se}$ ) to that on $\mathrm{Cr}$. The zero-gap behavior in spin down channel is the most important character. It suggests the inverted band structure around $\Gamma$, which is similar to the case in $\mathrm{HgSe}$ or $\mathrm{HgTe}^{108,109}$. 


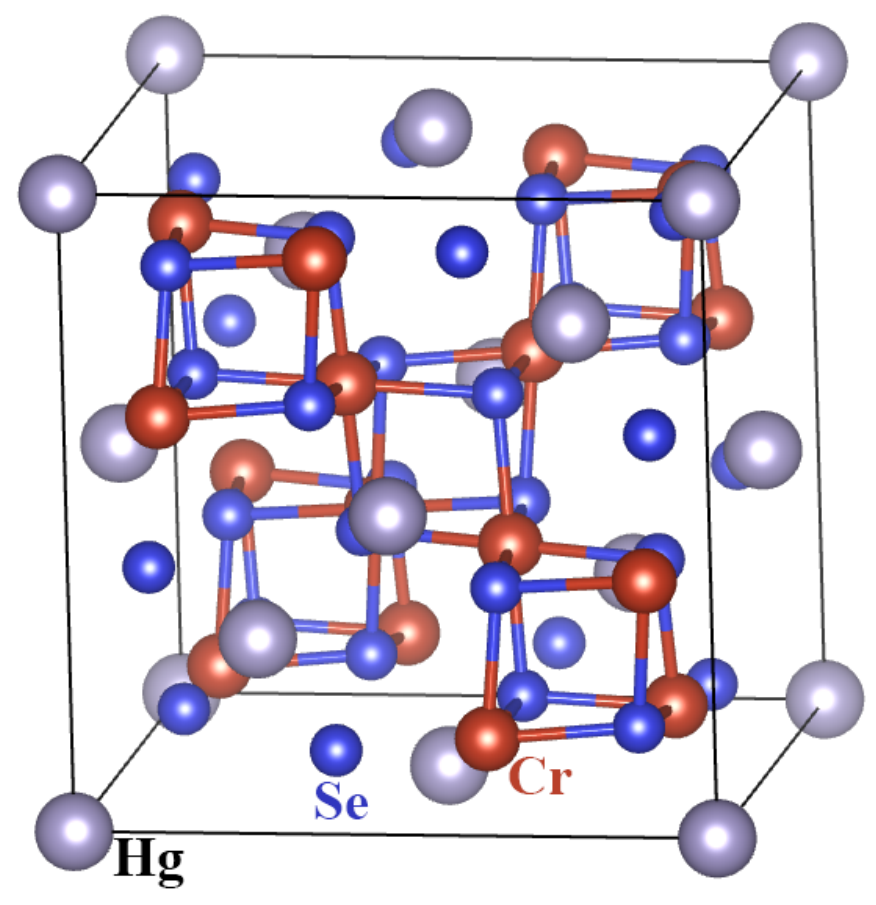

FIG. 10: (Color online) Crystal structure of spinel $\mathrm{HgCr}_{2} \mathrm{Se}_{4}$.
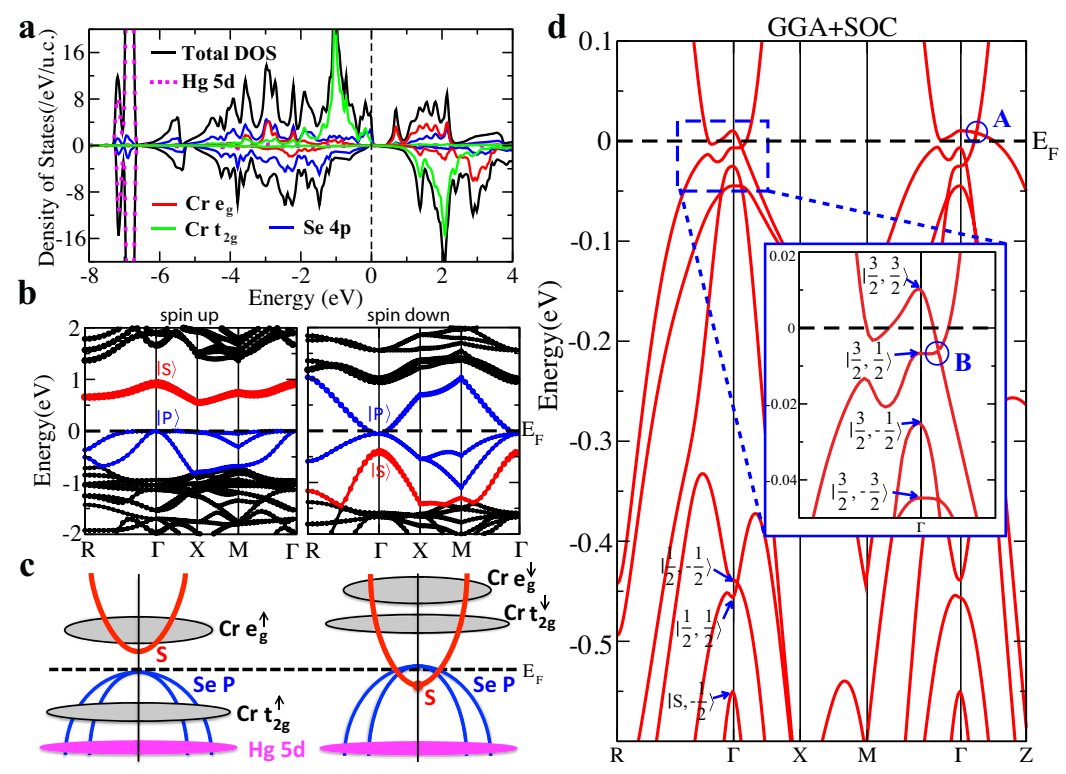

FIG. 11: (Color online) Electronic Structures of $\mathbf{H g C r}_{2} \mathbf{S e}_{4}$. (a) The total and partial density of states (DOS); (b) The band structures without SOC (showing the up and down spin parts separately); (c) The schematic understanding for the band-inversion, where the $|S\rangle$ state is lower than the $|P\rangle$ states in the down spin channel; (d) The band structure after including SOC (with majority spin aligning to the (001) direction). The low energy states at $\Gamma$ are indicated as explained in the main text. 
The four low energy states (eight after considering spin degree of freedom) at $\Gamma$ can be labelled as $\left|P_{x}\right\rangle,\left|P_{y}\right\rangle,\left|P_{z}\right\rangle$, and $|S\rangle$, which are linear combinations of atomic orbitals: $\left|P_{\alpha}\right\rangle \approx \frac{1}{\sqrt{8}} \sum_{i=1}^{8}\left|p_{\alpha}^{i}\right\rangle$, and $|S\rangle \approx 0.4 \sum_{j=1}^{2}\left|s^{j}\right\rangle+0.24 \sum_{k=1}^{4}\left|d_{t_{2 g}}^{k}\right\rangle$, where $\alpha=x, y, z$ and $i, j, k$ runs over $\mathrm{Se}, \mathrm{Hg}, \mathrm{Cr}$ atoms in the unit cell; $|s\rangle,\left|p_{\alpha=x, y, z}\right\rangle,\left|d_{t_{2 g}=x y, y z, z x}\right\rangle$ are the atomic orbitals of each atom. Taking these four states as basis, it is found to be the same situation in HgSe or HgTe. The only difference is the presence of exchange splitting in $\mathrm{HgCr}_{2} \mathrm{Se}_{4}$. The band inversion, $|S, \downarrow\rangle$ being lower than $|P, \downarrow\rangle$ at $\Gamma$ as shown in Fig. 11(c), is due to the following two factors. Firstly, the $\mathrm{Hg}-5 d$ states are very shallow (located at about $-7.0 \mathrm{eV}$ in Fig. 11(a)) and its hybridization with Se- $4 p$ states pushes the antibonding Se- $4 p$ states higher, similar to that in HgSe. Secondly, the hybridization between unoccupied Cr-3 $d^{\downarrow}$ and Hg- $6 s^{\downarrow}$ states in the down spin channel pushes the Hg-6s $s^{\downarrow}$ state lower in energy (Fig. 11 (b) and (c)). Therefore, the $|S, \downarrow\rangle$ is about $0.4 \mathrm{eV}$ lower than $|P, \downarrow\rangle$ states at $\Gamma$, and it is further enhanced to be $0.55 \mathrm{eV}$ in the presence of SOC. It is important to check the correlation effect beyond GGA. It has been shown that semiconducting $\mathrm{CdCr}_{2} \mathrm{~S}_{4}$ and $\mathrm{CdCr}_{2} \mathrm{Se}_{4}$ can be well described by the $\mathrm{LDA}+U$ calculations with effective $U$ around $3.0 \mathrm{eV}^{110,111}$. The same $\mathrm{LDA}+U$ calculations for $\mathrm{HgCr}_{2} \mathrm{Se}_{4}$ show that the band-inversion remains unless the $U$ is unreasonably large $(>8.0 \mathrm{eV})$.

Considering SOC, the new low energy eigen states at $\Gamma$ are given as $\left|\frac{3}{2}, \pm \frac{3}{2}\right\rangle,\left|\frac{3}{2}, \pm \frac{1}{2}\right\rangle$, $\left|\frac{1}{2}, \pm \frac{1}{2}\right\rangle$, and $\left|S, \pm \frac{1}{2}\right\rangle$, which can be constructed from the $|P\rangle$ and $|S\rangle$ states. Using this effective hamiltonian, the low energy band structures of $\mathrm{HgCr}_{2} \mathrm{Se}_{4}$ can be well reproduced. Due to the exchange splitting, the eight states at $\Gamma$ are all energetically separated, with the $\left|\frac{3}{2}, \frac{3}{2}\right\rangle$ having the highest energy, and the $\left|S,-\frac{1}{2}\right\rangle$ being the lowest. Several band-crossings are observed as shown in the band structure of Fig. 11(d). Among them, two kinds of bandcrossings (called A and B) are important for the states very close to the Fermi level. The crossing-A gives two points located at $k_{z}= \pm k_{z}^{c}$ along the $\Gamma-Z$ line, and the trajectory of crossing-B is a closed loop surrounding $\Gamma$ point in the $k_{z}=0$ plane, as schematically shown in Fig. 12(a). For the 2D planes with fixed $k_{z}\left(k_{z} \neq 0\right.$ and $\left.k_{z} \neq \pm k_{z}^{c}\right)$, the band structures are all gapped in the sense that a curved Fermi level is defined. Thus, the Chern number $C$ for each $k_{z}$-fixed plane can be evaluated. It is found that $C=0$ for the planes with $k_{z}<-k_{z}^{c}$ or $k_{z}>k_{z}^{c}$, while $C=2$ for the planes with $-k_{z}^{c}<k_{z}<k_{z}^{c}$ and $k_{z} \neq 0$. The crossing-A points locate at the phase boundary between $C=2$ and $C=0$ planes, i.e. $k_{z}= \pm k_{z}^{c}$ plane, and they are topologically unavoidable Weyl nodes. On the other hand, however, the 


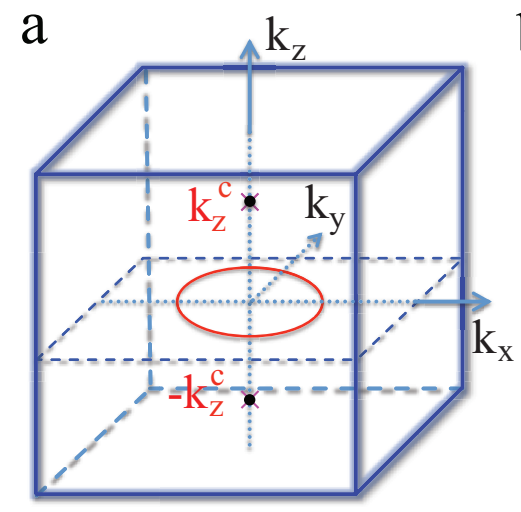

b
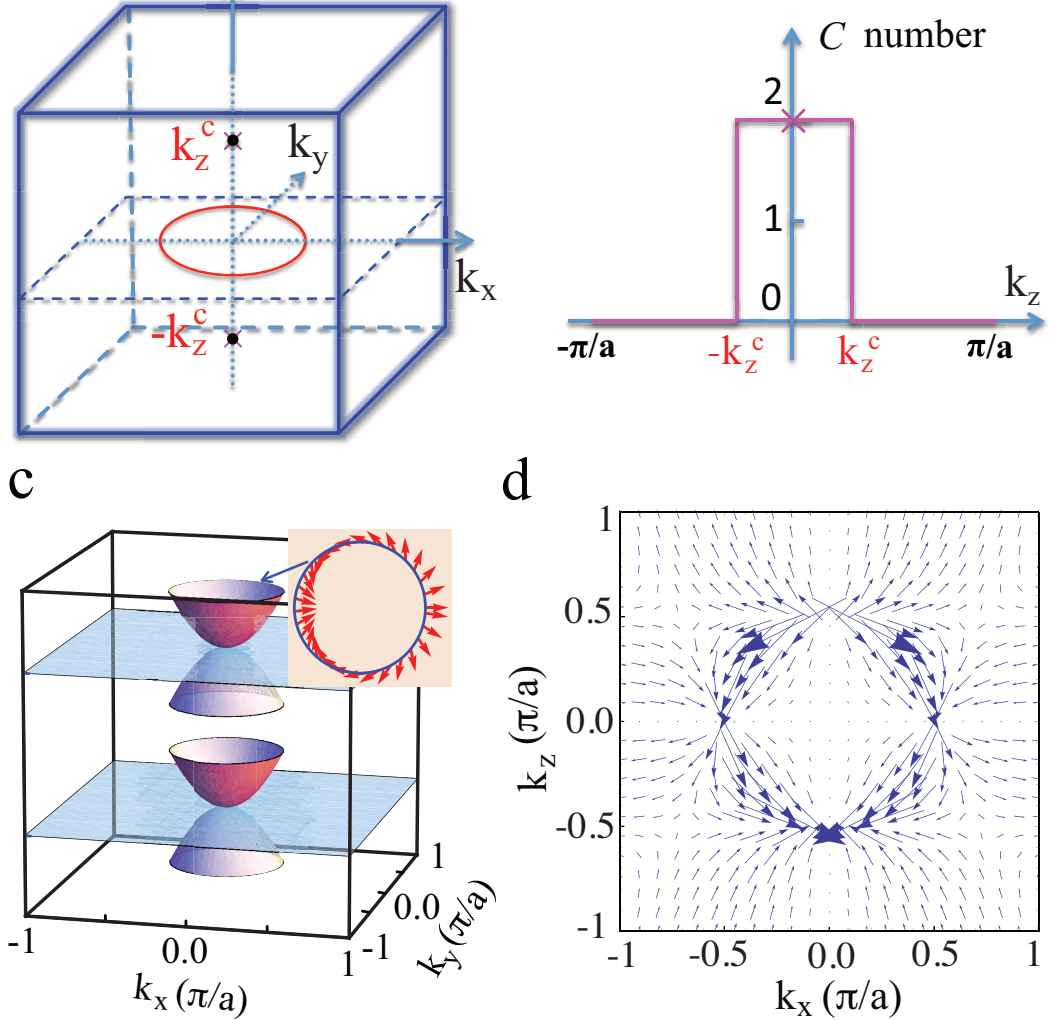

FIG. 12: (Color online) Weyl nodes and Berry flux in momentum space of

$\mathbf{H g C r}_{2} \mathbf{S e}_{4}$. (a) The band-crossing points $\pm k_{z}^{c}$; (b) The Chern number of occupied states in 2D planes perpendicular to $k_{z}$ axis; (c) The schematic plot of the band dispersion around the Weyl nodes in the $k_{z}= \pm k_{z}^{c}$ plane, and the inset shows the chiral spin texture.

(d) The Berry flux evaluated as Berry's curvature in the $\left(k_{x}, k_{z}\right)$ plane with $k_{y}=0$.

crossing-B points forming the closed loop in $k_{z}=0$ plane are just accidental and it is due to the presence of crystal mirror symmetry with respect to the $k_{z}=0$ plane. The crossing-B is not as stable as crossing- $\mathrm{A}$ in the sense that it can be changed by changing of the crystal symmetry. This node-line becomes a hot topic recently as we will discuss in the next section.

Considering the minimum two basis $\left|\frac{3}{2}, \frac{3}{2}\right\rangle$ and $\left|S,-\frac{1}{2}\right\rangle$ which catch the band-inversion nature, the $8 \times 8$ Hamiltonian can be downfolded into an effective $2 \times 2$ model by only keeping these two states and integrating out all the other bands, which reads

$$
H_{e f f}=\left[\begin{array}{cc}
M & D k_{z} k_{-}^{2} \\
D k_{z} k_{+}^{2} & -M
\end{array}\right] .
$$

Here $k_{ \pm}=k_{x} \pm i k_{y}$, and $M=M_{0}-\beta k^{2}$ is the mass term up to the second order. $M_{0}>0$ 

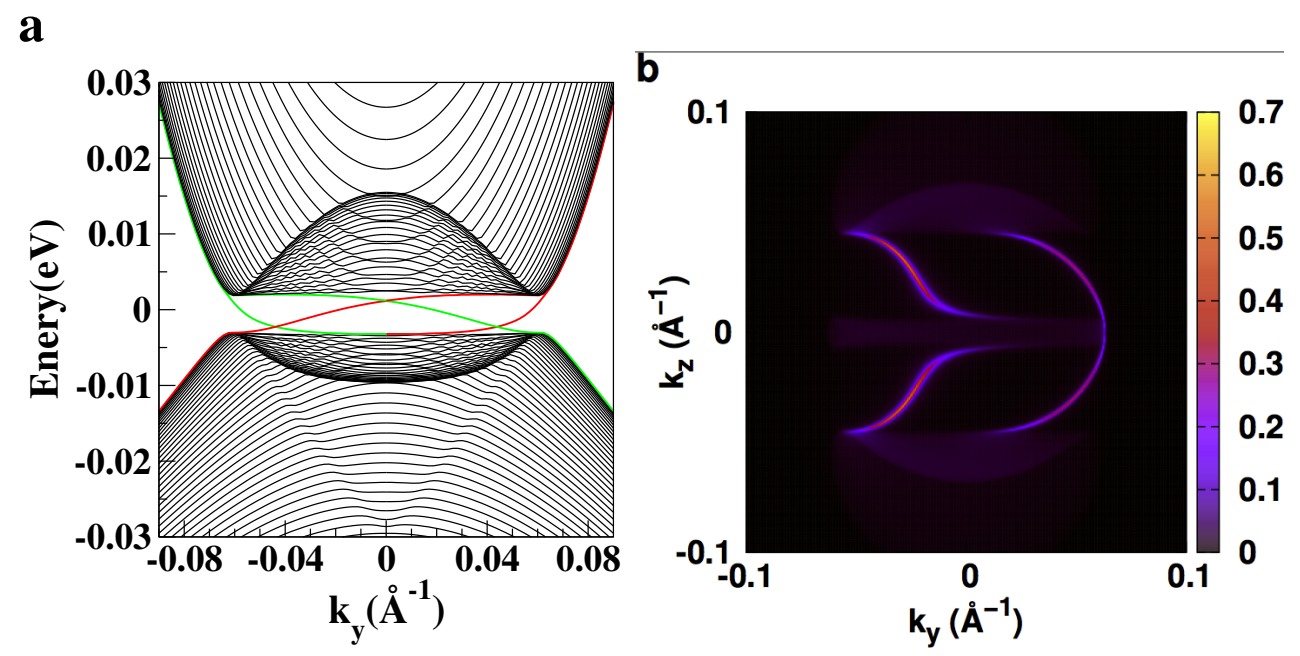

FIG. 13: (Color online) Edge states and Fermi arcs of $\mathbf{H g C r}_{2} \mathbf{S e}_{4}$ • (a) The calculated edge states for the plane with $k_{z}=0.06 \pi$. A ribbon with two edges is used, and there are two edge states for each edge (because $C=2$ ). The states located at different edges are indicated by different colors. (b) The calculated Fermi arcs for the $\left(k_{y}, k_{z}\right)$ side surface.

and $\beta>0$ are the condition to ensure the band inversion. Since the two basis have opposite parity, the off-diagonal element has to be odd in $k$. The $k_{ \pm}^{2}$ term is necessary to conserve the angular moment along $z$-direction. Therefore, to the leading order, the $k_{z} k_{ \pm}^{2}$ is the only possible form for the off-diagonal element. This two level hamiltonian has eigen values $E(k)= \pm \sqrt{M^{2}+D^{2} k_{z}^{2}\left(k_{x}^{2}+k_{y}^{2}\right)^{2}}$. Two gapless solutions: one is the degenerate points along the $\Gamma-Z$ line with $k_{z}= \pm k_{z}^{c}= \pm \sqrt{M_{0} / \beta}$; the other is a circle around the $\Gamma$ point in the $k_{z}=0$ plane determined from the equation $k_{x}^{2}+k_{y}^{2}=M_{0} / \beta$. These are consistent with the first-principles calculations. The presence of $k_{ \pm}^{2}$ in the off-diagonal term ${ }^{112}$ makes the Chern number $C$ being 2 for the planes of $-k_{z}^{c}<k_{z}<k_{z}^{c}$ except the $k_{z}=0$ plane where node-line exists. The in-plane band dispersions near the Weyl nodes at $k_{z}= \pm k_{z}^{c}$ are quadratic rather than linear, with a phase of $4 \pi$ for the chiral spin texture as shown in Fig. 12(c). The two Weyl nodes located at $\pm k_{z}^{c}$ have opposite chirality, and they form a single pair of magnetic monopoles carrying gauge flux in $\vec{k}$-space as shown in Fig. 12(d). The band-crossing loop in the $k_{z}=0$ plane is not topologically unavoidable, however, its existence requires that all gauge flux in the $k_{z}=0$ plane (except the loop itself) must be zero.

This Chern semi-metal state realized in $\mathrm{HgCr}_{2} \mathrm{Se}_{4}$ will lead to novel physical consequences, which can be measured experimentally. The 8-bands Kane model fitted from first-principles 

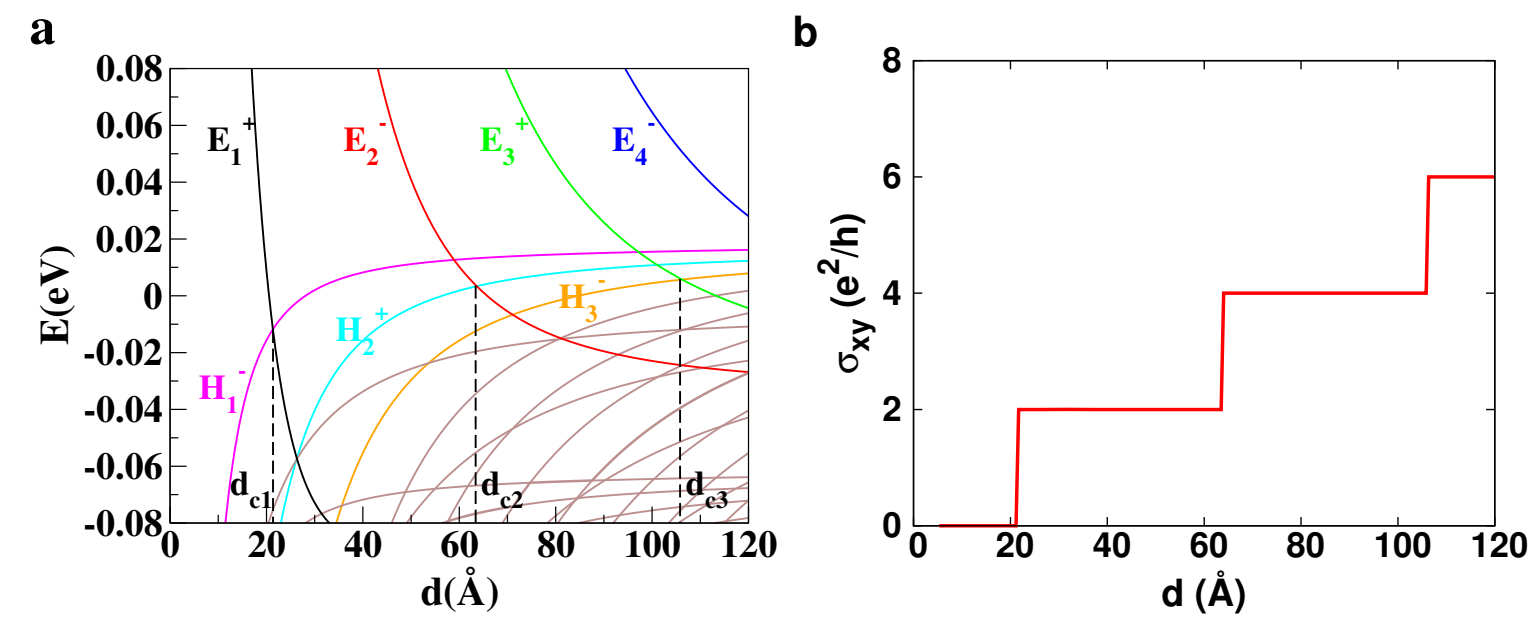

FIG. 14: (Color online) Quantized Anomalous Hall effect in $\mathbf{H g C r}_{2} \mathbf{S e}_{4}$ thin film.

(a) The subband energy levels at $\Gamma$ point as function of film thickness. (b) The Hall conductance as function of film thickness.

calculations is used for the numerical demonstration. First, each $k_{z}$-fixed plane with non-zero Chern number can be regarded as a 2D Chern insulator, and there must be chiral edge states for such plane if an edge is created. By Replacing $k_{x}$ with $-i \hbar \partial_{x}$ and using open boundary condition along $\mathrm{x}$ (or $\mathrm{y}$ ) direction, one can obtain the edge states for each fixed- $k_{z}$. The number of edge states is two for the case of $C=2$ as shown in Fig. 13(a), or zero for the case of $C=0$. If the chemical potential is located within the gap, only the chiral edge states can contribute to the Fermi surface, which are isolated points for each Chern insulating plane but nothing for the plane with $C=0$. Therefore the trajectory of such points in the $\left(k_{x}, k_{z}\right)$ surface or $\left(k_{y}, k_{z}\right)$ surface form non-closed fermi arcs, which can be measured by ARPES as shown in Fig. 13 (b). This is very much different from the conventional metals, where the Fermi surfaces must be either closed or interrupted by the Brillouin zone boundary. The possible Fermi arcs has been recently discussed from a view point of accidental degeneracy for pyrochlore iridates ${ }^{47}$. In general, any magnetic system with band-crossing nodes at Fermi level may show Fermi arcs on its surface. As demonstrate in Fig. 13, the calculated Fermi arcs for $\mathrm{HgCr}_{2} \mathrm{Se}_{4}$ not only end at $k_{z}= \pm k_{z}^{c}$, but also are interrupted by the $k_{z}=0$ plane, where the Node-line exists. Nevertheless, for WSM the Fermi arcs should be stable because the Weyl nodes are topologically unavoidable.

The QAHE, however, is an unique physical consequence characterizing the WSM nature in ferromagnetic $\mathrm{HgCr}_{2} \mathrm{Se}_{4}$ quantum-well structure. ${ }^{23}$ For $2 \mathrm{D}$ band insulators, the transverse 
Hall conductance should be quantized as $\sigma_{x y}=C \frac{e^{2}}{h}$, where $C$ is the Chern number. Such a quantum Hall effect without magnetic field has been long-pursued, ${ }^{4,21,23,24}$ but only achieved experimentally in 2013 in magnetic ion doped topological insulator films as theoretically predicted in $2010 .{ }^{23-25} \mathrm{In} \mathrm{HgCr}_{2} \mathrm{Se}_{4}$, considering the $k_{z}$-fixed planes, the Chern number $C$ is non-zero for limited regions of $k_{z}$ due to the band inversion around $\Gamma$ as discussed above. In the quantum well structure, the low energy states around $\Gamma$ are quantized into subbands, with $\left|H_{n}\right\rangle$ and $\left|E_{n}\right\rangle$ for hole and electron subbands, respectively. All the subbands' energy level change as function of film thickness. As shown in Fig. 14, when the thickness of the film is very thin the band inversion in the bulk band structure will be removed entirely by the finite size effect. With the increment of the thickness, the band inversion among these subbands restores, which results in jumps in the Chern number or the Hall coefficient $\sigma_{x y}{ }^{22,23}$. As shown in Fig. 14(b), the critical thickness of the film is around $21 \AA$. The subsequent jumps of $\sigma_{x y}$ is in unit of $2 e^{2} / h$ due to the change of 2 in Chern number. This brings an interesting way to realize high plateau quantum Hall conductivity of 2 in QAHE, being distinguished from present experimentally achievable magnetic doped TI system. ${ }^{23}$ In fact, the strong anomalous Hall effect has been observed for the bulk samples of $\mathrm{HgCr}_{2} \mathrm{Se}_{4}{ }^{102}$. This is in sharp contrast to pyrochlore iridates, where the anomalous Hall effect should be vanishing due to the AF ordering.

Inspired by this theoretical proposal, recent detailed transport studies have revealed that the ground state of $\mathrm{HgCr}_{2} \mathrm{Se}_{4}$ has nearly full spin-polarized current in its $s$-orbital conduction band, which is a strong evidence for its half-metallicity. ${ }^{113}$

\section{2: Non-magnetic Weyl Semimetal: TaAs family}

In addition to the two magnetic WSMs, namely pyrochlore iridate $R n_{2} \mathrm{Ir}_{2} \mathrm{O}_{7}{ }^{47}$ and spinel $\mathrm{HgCr}_{2} \mathrm{Se}_{4}{ }^{59}$ introduced above, other proposals involve a fine-tuned multilayer structure composed of normal insulator and magnetically doped TIs $^{60}$ or just magnetic ion doped TIs. ${ }^{114}$ All of them involve magnetic compounds and the spin degeneracy of the bands is removed by breaking time reversal symmetry. However, these proposals are still waiting for experimental confirmation.

As mentioned above, WSM can also be generated by breaking the spatial inversion symmetry only but keeping time-reversal symmetry. This is the nonmagnetic and noncentrosymmetric WSM. Compared with the magnetic WSM, nonmagnetic WSM has the following advantages. 1) it can be more easily studied by ARPES measurement since the alignment of 
magnetic domains is not required; 2) without the spin exchange field, the unique structure of Berry curvature related with magnetic monopoles leads to very unusual transport properties under external magnetic field. Such properties are not mixed by the self-magnetization of the magnetic sample.

There have been several proposals for WSM in system without inversion symmetry, such as a superlattice composed of alternatively stacking normal and topological insulators, ${ }^{60,115}$ elemental Tellurium or Selenium crystal under proper pressure, ${ }^{116}$ the fine-tuned solid solution of $A \mathrm{Bi}_{1-x} \mathrm{Sb}_{x} \mathrm{Te}_{3}(A=\mathrm{La} \text { and } \mathrm{Lu})^{117}$ around the topological transition points. ${ }^{51,52}$ There is none of the above has been realized experimentally due to the following possible problems: 1) very fine control, such as quantum well thickness and doping concentration, is required in sample preparation; 2) applying pressure makes the other measurement very difficult.

In the end of 2014, our collaborators and us have posted the theoretical prediction that TaAs,TaP, NbAs and NbP single crystals are natural WSM and possess 12 pairs of Weyl points. ${ }^{118}$ Compared with the other proposals, this family of materials are completely stoichiometric without any additional doping, external strain or pressure. High quality sample can be easily obtained and experimental verification becomes quite easier.

Unlike in the case of pyrochlore iridates and $\mathrm{HgCr}_{2} \mathrm{Se}_{4}$, inversion symmetry is kept and the appearance of Weyl points can be immediately inferred from the product of the parities at all the time reversal invariant momenta (TRIM) ${ }^{119-121}$ For the TaAs family, parity is no longer a good quantum number. It is found that the appearance of Weyl points can be inferred by analyzing the mirror Chern numbers $(\mathrm{MCN})^{46,122}$ and $Z_{2}$ indices ${ }^{5,119,123,124}$ for the four mirror and time reversal invariant planes in the BZ. Similar as many other topological materials, the WSM phase in this family is also induced by band inversion, which, in the absence of spin-orbit coupling (SOC), leads to nodal rings in the mirror plane. Once SOC is turned on, each nodal ring will be gapped with the exception of three pairs of Weyl points, which lead to fascinating physical properties including the complicated Fermi arc structures on surface.

The experimental crystal structure of TaAs ${ }^{125}$ is shown in Fig. 15. It has body-centeredtetragonal structure with nonsymmorphic space group $I 4_{1} m d$ (No. 109) lacking inversion symmetry. The measured lattice constants are $a=b=3.4348 \AA$ and $c=11.641 \AA$. Both Ta and As are at $4 a$ Wyckoff position $(0,0, u)$ with $u=0$ and 0.417 for Ta and As, respectively. To calculate the topological invariant such as MCN and surface states of TaAs, we have 

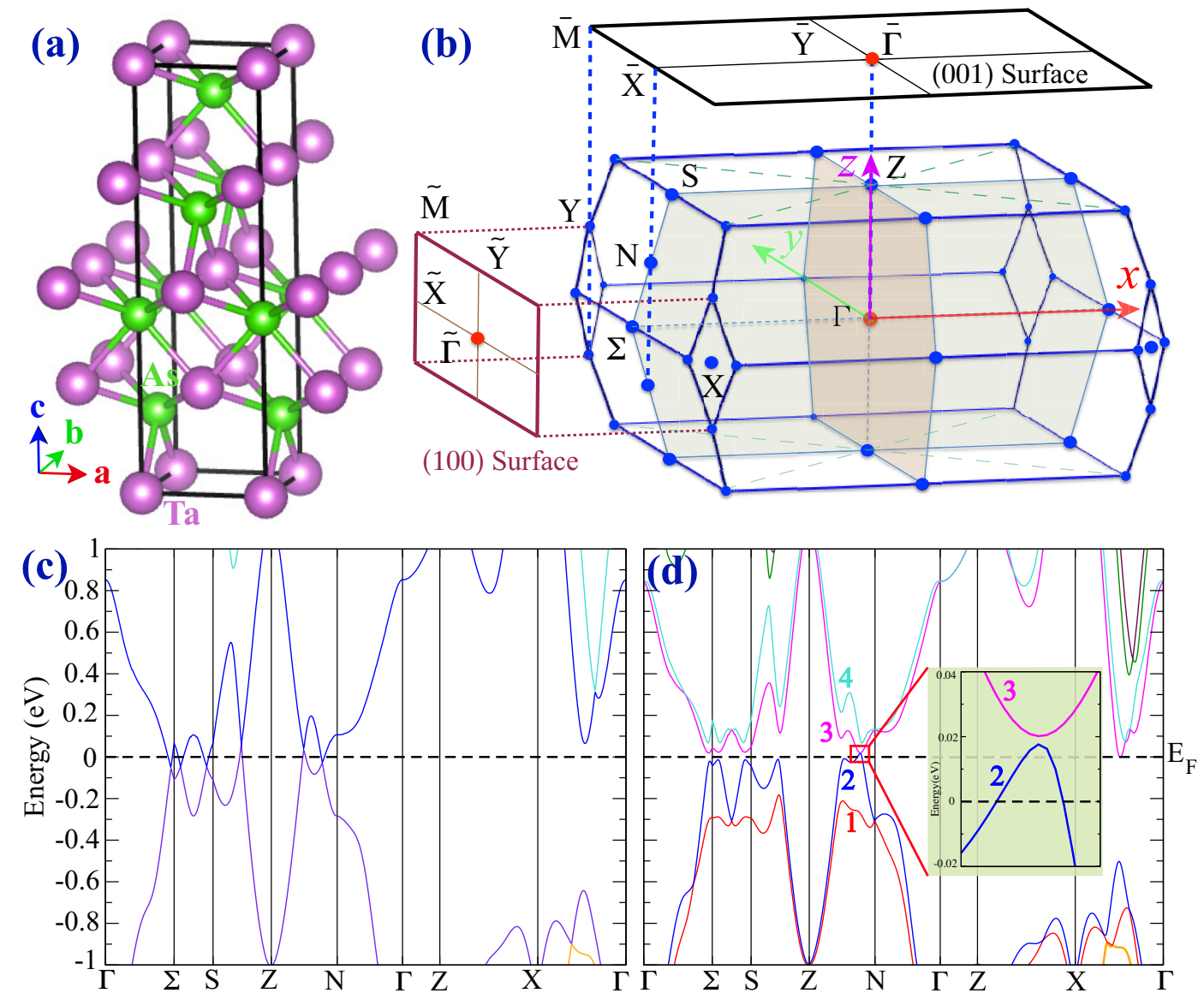

FIG. 15: Crystal structure and Brillouin zone (BZ). (a) The crystal symmetry of TaAs; (b) The bulk BZ and the projected surface BZ for both (001) and (100) surfaces; (c) The band structure of TaAs calculated by GGA without including the spin-orbit coupling; (d)The band structure of TaAs calculated by GGA with the spin-orbit coupling.

generated atomic-like Wannier functions for Ta $5 d$ and As $4 p$ orbitals using the scheme described in Ref. 126

The band structure of TaAs within GGA without including SOC is shown in Fig. 15(c). There is clear band inversion and multiple band crossing features near the Fermi level along ZN, ZS and $\Sigma$ S paths. In Fig.15(b), the shadowed planes are two mirror planes, namely $M_{x}, M_{y}$, while the dashed lines indicate two glide mirror planes, namely $M_{x y}, M_{-x y}$. The plane spanned by $\mathrm{Z}, \mathrm{N}$ and $\Gamma$ points is invariant under mirror $M_{y}$ and the energy bands within the plane can be labeled by mirror eigenvalues \pm 1 . Further analysis shows that the two bands that cross the $\mathrm{Z}$ to $\mathrm{N}$ line belong to opposite mirror eigenvalues and hence the crossing between them is protected by mirror symmetry. Similar band crossings can also be 
found along other high symmetrical lines in the ZN these band crossing points form a "nodal ring" in the ZN $\Gamma$ plane as shown in Fig.16(b). Unlike for the situation in ZN plane, in the two glide mirror planes $\left(M_{x y}\right.$ and $\left.M_{-x y}\right)$, the band structure is fully gaped with the minimum gap around $0.5 \mathrm{eV}$.

The bands near the Fermi energy are mainly formed by Ta $5 d$ orbitals, which have quite strong SOC. SOC brings dramatic changes in the band structure near Fermi level, as plotted in Fig. 15(d). It seems that the previous band crossings on the nodal rings in ZN are all gaped. Detailed symmetry analysis reveals that the bands "2" and "3" in Fig.15(d) belong to opposite mirror eigenvalues, indicating the almost touching point along the ZN line is completely accidental. In fact there is a small gap of roughly $3 \mathrm{meV}$ between bands "2" and " 3 " as illustrated by the inset of Fig.15(d). Therefore, the ZNC plane becomes fully gapped with SOC included.

There are three critical questions to be addressed to convincingly identify TaAs as a WSM. 1) Do Weyl nodes really exist? 2) How many of them? 3) Where are they? Since Weyl nodes are at general $k$-points with accidental double degenerate eigen energy, numerical method always has error due to discrete sampling of 3D momentum space. No matter how dense the sampling grid is used, finite numerical error exists and brings difficulty in identifying the existence of massless Dirac cone like band structure. Some efficient way mathematically rigorous is necessary to make final conclusion.

Since TaAs has no inversion center, the widely used and most simplified parity configuration method ${ }^{119-121,124}$ can not be applied to identify the existence of WSM. Another strategy is thus developed in Ref. 118. As previously mentioned, the space group of the material provides two mirror planes $\left(M_{x}\right.$ and $\left.M_{y}\right)$, where MCN can be defined. If a full gap exists for the entire BZ, the MCN would directly reveal whether this system is a topological crystalline insulator $^{45}$ or not. Interestingly, if the system is not fully gaped MCN is still useful to find out whether the material hosts Weyl points in the BZ or not. Besides the two mirror planes, there are two additional glide mirror plane $\left(M_{x y}\right.$ and $\left.M_{-x y}\right)$. MCN is not well defined for the glide mirror planes, but $Z_{2}$ index is still well defined here since these two planes are time reversal invariant and electronic states inside of them are insulator. The Wilson loop method $^{44,127}$ is applied to calculate the MCNs for the two mirror planes and $Z_{2}$ indices for the two glide mirror planes. To determine the MCNs for the mirror plane $M_{x}$, Wilson loops along $k_{x}$ direction with fixed $k_{z}$ are defined. All the occupied bands at $k$-points in this plane 


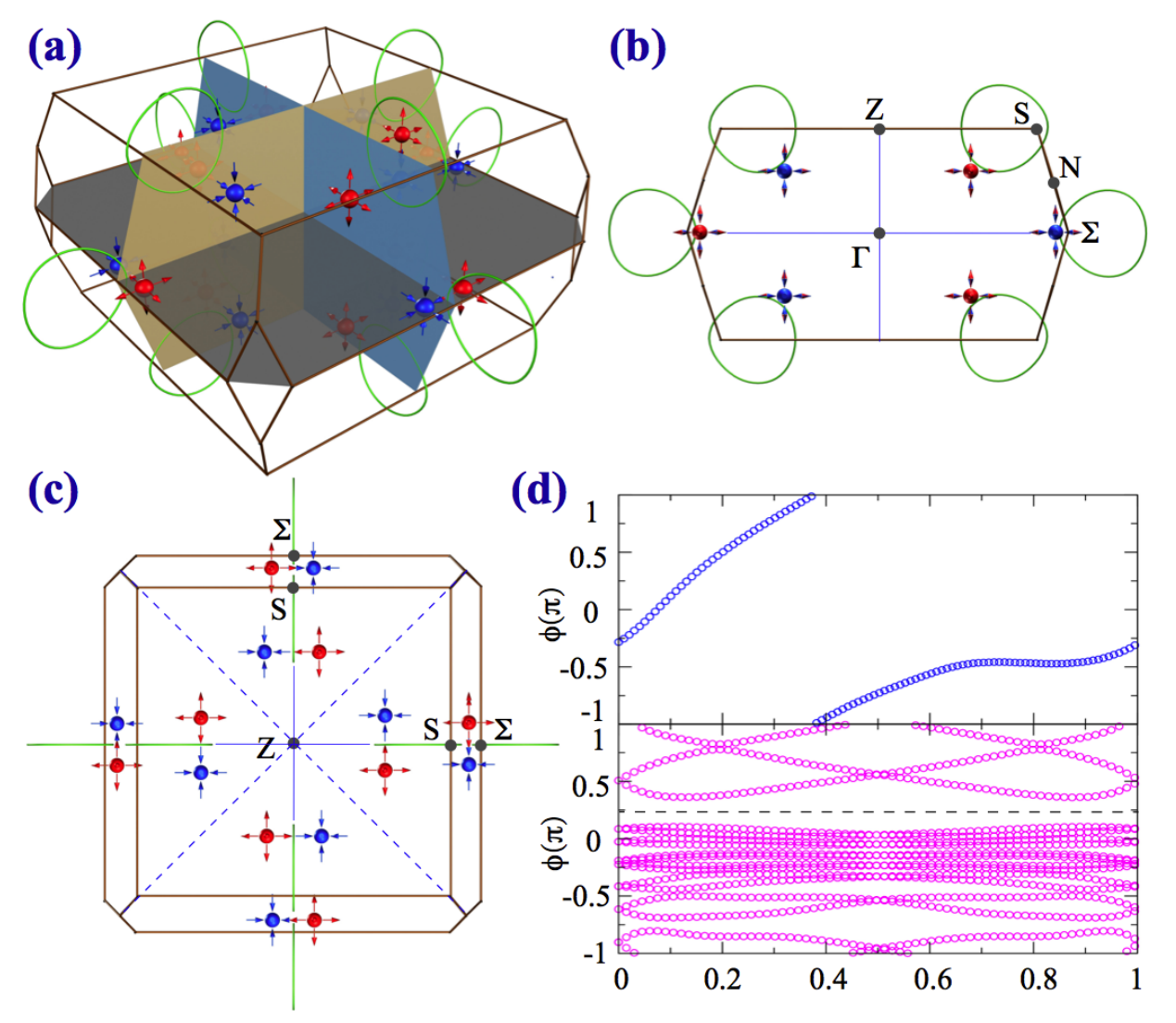

FIG. 16: Nodal rings and Weyl points distribution, as well as $Z_{2}$ and MCN for mirror planes. (a) 3D view of the nodal rings (in the absence of SOC) and Weyl points (with SOC) in the BZ; (b) Side view from [100] and (c) top view from [001] directions for the nodal rings and Weyl points. Once the SOC is turned on, the nodal rings are gapped and give rise to Weyl points off the mirror planes; (d) top panel: Flow chart of the average position of the Wannier centers obtained by Wilson loop calculation for bands with mirror eigenvalue $i$ in the mirror plane ZN ; bottom panel: The flow chart of the Wannier centers obtained by Wilson loop calculation for bands in the glide mirror plane ZХГ. There is no crossing along the reference line (the dashed line) indicating the $Z_{2}$ index is even.

can be classified into two groups according to their eigenvalues under mirror operation, $i$ or $-i$. For the bands having eigenvalue $i$, the evolution of Berry phases along periodic $k_{z}$ direction can be obtained and the MCN is simply its winding number. In Fig. 16(d), it is clear that MCN is one for ZN $\mathrm{ZN}$ plane $\left(M_{y}\right)$ and $Z_{2}$ index is even for ZX厂 plane $\left(M_{x y}\right)$. Thus, for the (001) surface, which is invariant under the $M_{y}$ or $M_{x}$ mirror, there will appear non-trivial helical edge modes due to non-zero MCN in ZNT plane, which generates a single 
pair of FS cuts along the projective line of the ZN $\Gamma$ plane, i.e. the $x$ - or $y$-axis in Fig. 16(c). Whether these Fermi cuts will eventually form a single closed Fermi circle or not depends on the $Z_{2}$ index for the two glide mirror plane, which are projected to the dashed blue lines in Fig. 16(c). Since the $Z_{2}$ indices for the glide mirror planes are trivial, there are no protected helical edge modes along the projective lines of the glide mirror planes, the dashed blue lines in Fig. 16(c), and the Fermi level cuts along the $x$ - or $y$-axis in Fig. 16(c) must end somewhere between the $x$ - or $y$ - axis and the diagonal lines. That means they must be Fermi arcs, ending at the surface projection of Weyl points in the bulk band structure of TaAs.

The existence of Weyl points is confirmed. The left questions are the total number and the exact positions of them. As mentioned above, this is a hard task since the Weyl points are located at generic $k$ points without any symmetry. For this purpose, we calculate the integral of the Berry curvature on a closed surface in $k$-space, which equals to the net chirality of the Weyl nodes enclosed by the given compact surface. Due to the four fold rotational symmetry and mirror planes that characterize TaAs, one only needs to search for the Weyl points within the reduced BZ, i.e., one eighth of the whole BZ. The total chirality or monopole charge enclosed in the whole reduced BZ is found to be one. This guarantees the existence of, and odd number of Weyl points in one eighth of BZ. To determine the exact location of each Weyl point, the reduced BZ is divided into very dense $k$-grid and the Berry curvature or the "magnetic field in momentum space"27,126,128 is calculated as shown in Fig. 17. From this, one can easily identify the precise positions of the Weyl points by searching for the "source" and "drain" points of the "magnetic field". Around the Weyl points, the hedgehog like Berry curvature is clearly shown in Fig. 17.

Therefore, there are totally twelve pairs of Weyl points in TaAs as illustrated in Fig. 16(a). All of them are in the vicinity of the nodal rings on two of the mirror invariant planes in the SOC-free case. There are two types of Weyl nodes. W2 are those located off the $k_{z}=0$ plane, totally eight pairs. W2 is those located exactly in the $k_{z}=0$ plane, totally four pairs. All W1 Weyl nodes are related by mirror symmetry, four-fold rotational symmetry, and time reversal symmetry, so does it for W2. However, W1 and W2 are not related by any symmetry and they can have different velocity matrix and located at different energy. For example, in TaAs, W2 nodes are about $2 \mathrm{meV}$ above the Fermi energy and form eight tiny hole pockets, while the W1 nodes are about $21 \mathrm{meV}$ below the Fermi level to form sixteen 


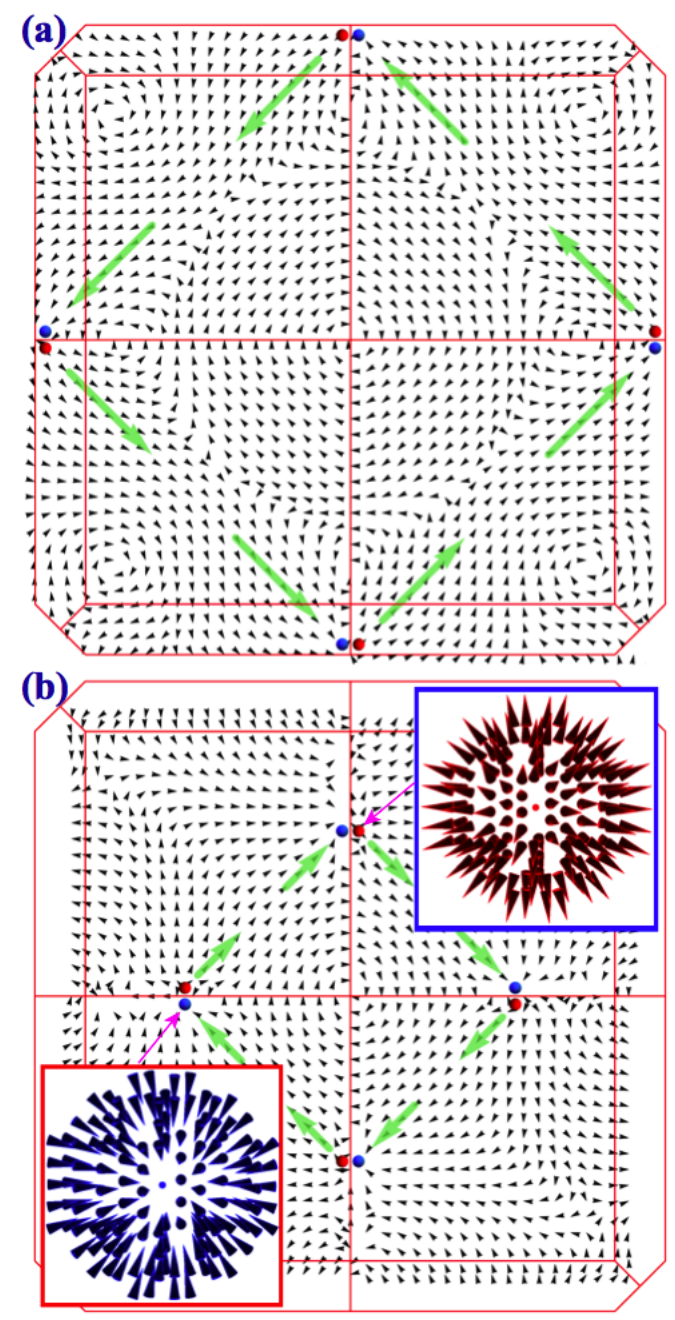

FIG. 17: Berry curvature from pairs of Weyl points. (a) The distribution of the Berry curvature for the $k_{z}=0$ plane, where the blue and red dots denote the Weyl points with chirality of +1 and -1 , respectively; (b) same with (a) but for $k_{z}=0.592 \pi$ plane. The insets show the 3D view of hedgehog like Berry curvature near the two selected Weyl points.

electron pockets. These values are obtained by dense $k$-grid band structure calculation based on tight-binding model from MLWFs of Ta $d$ and Te $p$ orbitals. SOC is included as onsite terms with $0.3 \mathrm{eV}$ for Ta $d$ and $0.2 \mathrm{eV}$ for Te $p$ as fitted from first-principles calculation with SOC included. The band structures for the other three materials TaP, NbAs and NbP are very similar. The precise positions of the Weyl points for all these materials are summarized in Table. I.

One of the hallmark of WSM is the Fermi arcs on the surface. ${ }^{39,47,59}$ In TaAs family, there 
TABLE I: The two nonequivalent Weyl points in the $x y z$ coordinates shown in Fig. 15(b). The position is given in unit of the length of $\Gamma-\Sigma$ for $x$ and $y$ and of the length of $\Gamma-Z$ for $z$.

\begin{tabular}{c|c|c}
\hline \hline & $\mathrm{W} 1$ & \multicolumn{1}{|c}{$\mathrm{W} 2$} \\
\hline $\mathrm{TaAs}$ & $(0.520,0.037,0.592)$ & $(0.949,0.014,0.0)$ \\
\hline $\mathrm{TaP}$ & $(0.499,0.045,0.578)$ & $(0.955,0.025,0.0)$ \\
\hline $\mathrm{NbAs}$ & $(0.510,0.011,0.593)$ & $(0.894,0.007,0.0)$ \\
\hline $\mathrm{NbP}$ & $(0.494,0.010,0.579)$ & $(0.914,0.006,0.0)$ \\
\hline \hline
\end{tabular}

are multiple Weyl points. When they are projected to one surface, some of them might be on top of each other. It is easy to see that the total number of surface modes at Fermi level crossing a closed circle in surface BZ must equal to the sum of the "monopole charge" of the Weyl points inside the 3D cylinder that projects to the given closed circle. Another important fact determining the behavior of the surface states is the MCN introduced in the above discussion, which limits the number of FSs cutting certain projection lines of the mirror plane as long as this mirror symmetry is still preserved on the surface.

By using the Green's function method ${ }^{44}$, the surface states for both (001) and (100) surfaces have been calculated as shown in Fig. 18 together with its Fermi surface. On the (001) surface, the crystal symmetry is reduced to $C_{2 v}$ leading to different behavior for the surface bands around $\bar{X}$ and $\bar{Y}$ points respectively. Along the $\bar{\Gamma}-\bar{X}$ or $\bar{\Gamma}-\bar{Y}$ lines, Fermi surface crosses it twice with opposite Fermi velocity. This satisfies the constraint from the MCN for $\Gamma Z N$ plane. In addition to the MCN, the possible "connectivity pattern" of the Fermi arcs on the surface has to link different projection points of the Weyl nodes with opposite chirality. However, for the (001) surface of TaAs, the connectivity pattern of the Fermi arcs that satisfy all the conditions discussed is not unique. Only the appearance of "Fermi arcs" on the (001) surface is guaranteed since all the projective points on the (001) surface are generated either by a single Weyl point or by two Weyl points with the same chirality. The Fermi arc connectivity pattern for (001) surface shown in Fig. 18(b) is obtained from our ab-initio calculation on a non-relaxed surface described by the TB model based on MLWFs of Ta $d$ and Te $p$ orbitals. Changes of surface potentials or the simple relaxation of the surface charge density might lead to modification of the Fermi arc connectivity pattern and result in topological Fermi arc phase transitions on the surface. 
The extremely long Fermi arcs which cross the zone boundary along the $\bar{X}$ to $\bar{M}$ line is quite interesting. Comparing with other proposed WSM materials, the Fermi arcs in TaAs families are much longer, which greatly facilitates their detection in experiments.

On the other hand, the surface states on (100) surface of TaAs are much more complicated as shown in Fig. 18(c) and (d). The biggest difference between them is that all the projected Weyl points on the (100) surface are formed by a pair of Weyl points with opposite chirality, which does not guarantee (but does not disallow) the existence of the Fermi arcs. The only constraint for the (100) surface states is the nonzero MCN of the $\Gamma Z N$ plane, which generates a pair of chiral modes along the $\bar{\Gamma} \bar{Y}$ line, the projection of the mirror plane, as illustrated in Fig.18(d).

After this theoretical perdition, our collaborators and us have reported several experimental works to confirm it. Totally, there are four hallmarks of WSM in TaAs family have been observed: 1) Fermi arcs on its (001) surface; ${ }^{129}$ 2) Weyl nodes in its bulk state; ${ }^{130}$ 3) negative magnetoresistivity due to "chiral anomaly";131 4) Spin texture of the Fermi arcs on (001) surface. ${ }^{132}$ The other experimental works appeared include Ref. 133-143. The topological superconductivity $^{144-147}$ is also expected for either DSM or WSM, and several experimental efforts $^{148-150}$ have been reported.

\section{Node-Line Semimetal}

In the former two sections, we have discussed the TSMs with isolated Dirac cone like nodes at or close to the Fermi level. However, there is another interesting electronic states having the nodes forming a closed ring or periodically continuous line in momentum space. Such kind of semimetal state now is known as topological nodal semimetal ${ }^{61}$ or topological NodeLine semimetal (NLSM). ${ }^{151}$ In 2011, Burkov et al. proposed that a fine tuned superlattice

of normal insulator and TI with broken TRS might realize NLSM. ${ }^{61}$ This is basically a model proposal rather than a practically realizable material candidate. In this section, we will introduce some realistic 3D compounds proposed to host NLSM. The underlying mechanism is also based on band inversion, which causes Node-Line when both TRS and IS are conserved and SOC is neglected for light element compounds.

\section{1: All Carbon Mackay-Terrones Crystal}

In 1992, A. L. Mackay and H. Terrones proposed that ${ }^{152}$ graphene can be extended to form 3D networks by placing graphitic tiles consisting of 4- to 8-membered rings onto the Schwarz minimal surface. Such kind of 3D all carbon allotrope is called as Mackay-Terrones 

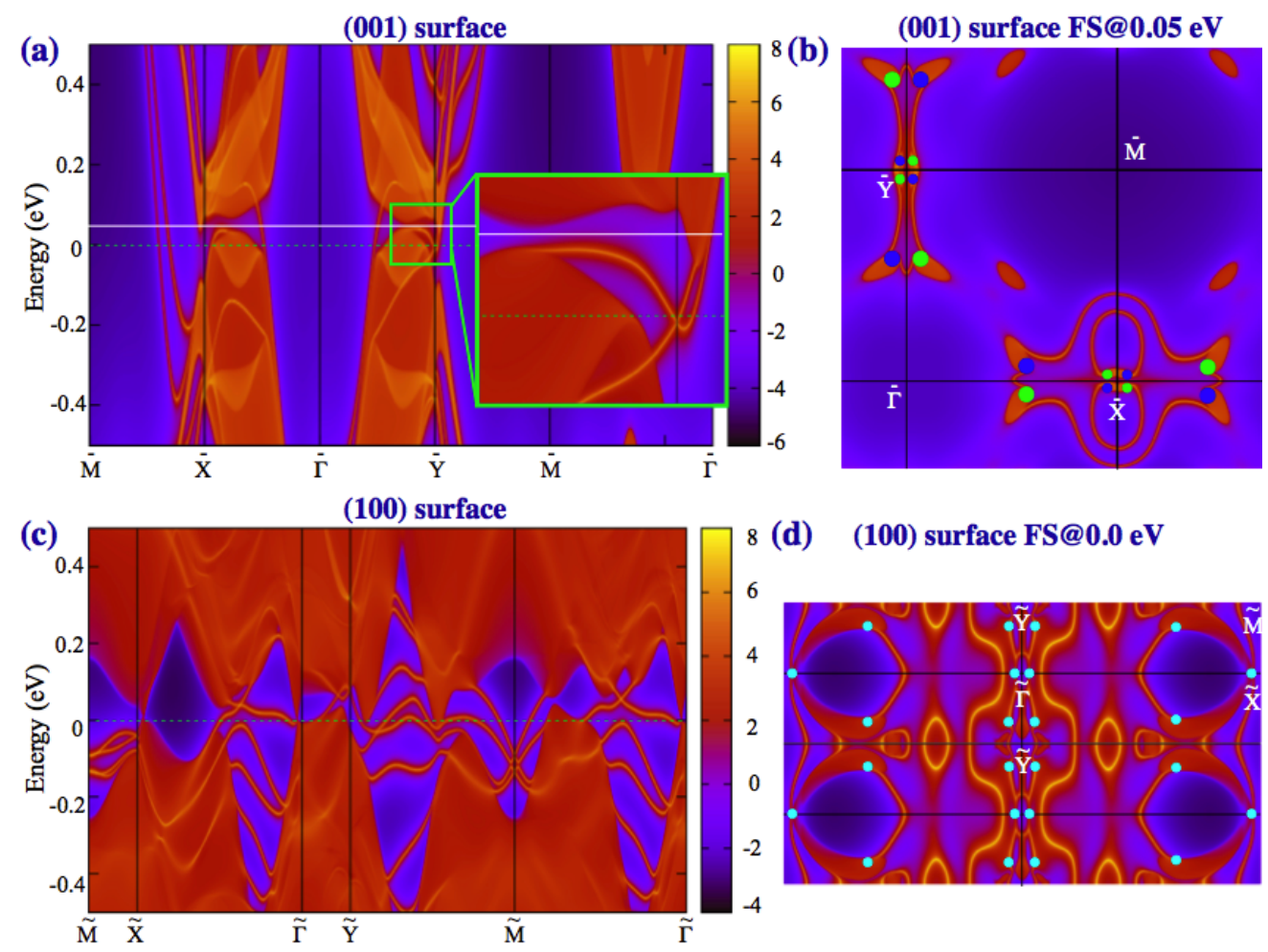

(d) (100) surface FS@0.0 eV

FIG. 18: Fermi arcs in the surface states. (a) The Surface states for (001) surface; (b) the corresponding fermi surfaces on (001) surfaces; (c) The Surface states for (100) surface. The dots illustrate the projective points of the bulk Weyl points on the surfaces, where the color represents the chirality of the Weyl points (blue for positive and green for negative), small dot represents the single projected Wely point, and large dot represents two Weyl points with same chirality projecting on top of each other. (d) the corresponding fermi surfaces on (100) surfaces. All the dots here are the projective points for a pair of Weyl points with opposite chirality.

crystal (MTC). Schwarz minimal surface is a 3D periodic minimal surface with its mean curvature $H=\left(k_{1}+k_{2}\right) / 2$ being zero and Gaussian curvature $\left(K=k_{1} k_{2}\right)$ being negative everywhere on it. The above $k_{1}$ and $k_{2}$ are the principal curvatures. There are various Schwarz minimal surfaces, such as primitive (P), diamond (D) and gyroid $(G)$ surface. One type of MTC based on P-surface is shown in Fig. 19. Different from $\mathrm{C}_{60}$-like fullerene, which has positive Gaussian curvature, MTC has negative Gaussian curvature and is periodically connected. It is shown such all-carbon MTC can host non-trivial electronic states, including topological node-lines and 3D Dirac points, which are distinct from its 2D counter material graphene. Similar node-lines have also been proposed in optimally tuned photonic crystal 

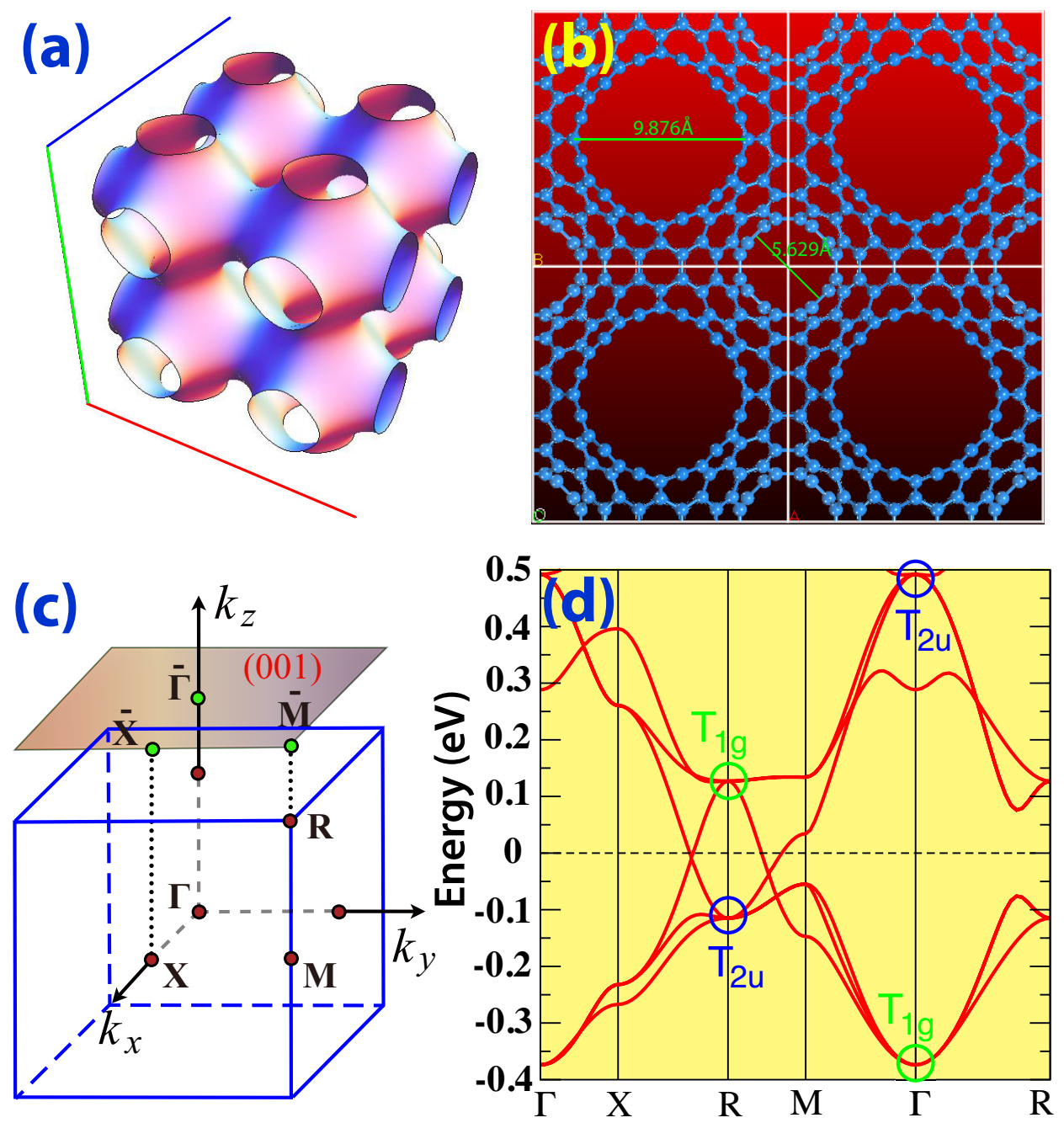

FIG. 19: (Color online) (a) The Schwarz minimal P-surface in $2 \times 2 \times 2$ supercell. (b) The top view of 6-1-1-p MTC in $2 \times 2$ supercell. (c) Bulk and (001)-surface Brillouin zone, as well as the high symmetrical k-points. (d) Band structure from the first-principles calculation. The two triply degenerated eigenstates at $\Gamma$ and $\mathrm{R}$ with $\mathrm{T}_{1 g}$ and $\mathrm{T}_{2 u}$ symmetrical representation are marked. The band inversion between them can be easily seen.

composed of gyroid, ${ }^{153}$ the Schwarz minimal G-surface.

Fig. 19 shows a stable structure with simple cubic lattice in $P m \overline{3} m$ space group. It contains 176 atoms per unit cell and is obtained by Tagami et al. in Ref. 154 and labeled as 6-1-1-p. The electronic band structure of this crystal, calculated based on the local density approximation (LDA), is shown in Fig. 19(d). It indicates this crystal is a semimetal with band crossings around the Fermi level, similar to the massless Dirac cone in graphene, but 

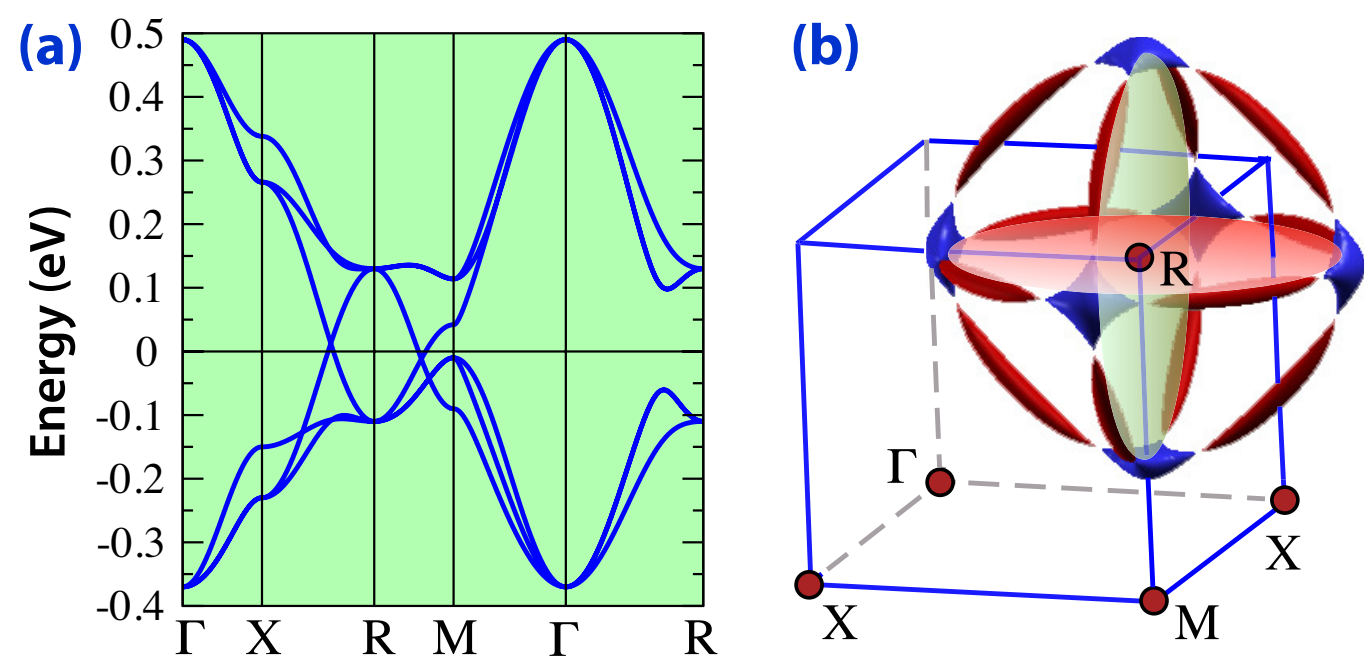

FIG. 20: (Color online) (a) Band structure from effective tight-binding model calculation, which reproduces all the features of Fig. 19(d). (b) Fermi surface consists of three lotus root like rings. These rings are centering $\mathrm{R}$ point and parallel to the $k_{x}=\frac{\pi}{a}, k_{y}=\frac{\pi}{a}$ and $k_{z}=\frac{\pi}{a}$ plane, respectively. They are formed by the electron pockets (blue) and hole pockets (red) connected by nodal points at Fermi energy.

they are essentially different. The occupied and unoccupied low energy bands are triplydegenerate at $\Gamma$, and have $\mathrm{T}_{1 g}$ (even parity) and $\mathrm{T}_{2 u}$ (odd parity) symmetry, respectively. Moving away from $\Gamma$ point, their degeneracy is lifted but at $R$ point, their degeneracy is recovered again. However, the odd and even eigenstates exchanges their energy ordering, which is a typical band inversion picture. Thus, the band inversion leads to the band crossings along both $X-R$ and $R-M$ paths as seen from Fig. 19(d). Including SOC will open a band gap on the nodal points and it becomes a $3 \mathrm{D}$ strong TI with $Z_{2}$ index $(1 ; 111)^{119,124}$ if the lower (upper) half of the anti-crossing bands are thought as occupied (unoccupied). However, the computed SOC splitting is very small, around $0.13 \mathrm{meV}$ or equivalent to $1.5 \mathrm{~K}$ temperature, which can be neglected when temperature is higher than $1.5 \mathrm{~K}$.

Without SOC, the most interesting thing found in MTC is that the band crossing points lead to nodal lines rather than nodal points. In fact, the band crossings form closed loops in the 3D momentum space, and generate three circular-like node-lines around the $R$ point, as shown in Fig. 20.

Detailed analysis indicates that these node-lines are protected by two factors. One is the 
coexistence of TRS and IS and the other is that the SOC is negligible. The cubic symmetry of MTC leads to three in-plane nodal rings. The node-lines are not necessarily to be flat in energy, i. e., the crossing points might be at different energy level at different $k$, which leads to compensated electron and hole pockets along the node-lines.

Different from other proposals for the topological node-lines, such as that in Ref. 61, the appearance of node-lines in MTC is very stable and does not require fine tuning of any parameter. This mechanism to generate topological node-lines in 3D materials only requires TRS, IS and negligible SOC, which can be easily applied to a large class of materials consisting of mainly the light elements.

This topologically stable node-line semimetal state can have nontrivial surface states. ${ }^{61,155-158}$ For the (001)-surface, the three node-line rings are projected to be a ring and two orthogonal diameter segments inside of it as shown in Fig. 21(a). The (001)-surface state is calculated based on a six-band TB model using both Green's function method and slabmodel method. ${ }^{44}$ There is a nearly flat surface band nestled inside of the projected node-line ring with its band width being about $40 \mathrm{meV}$ due to the particle-hole asymmetry. The peak-like surface density of states contributed by this nearly flat band is clearly shown in Fig. 21(b). This is proposed to be an important route to high temperature surface superconductivity. ${ }^{159,160}$ The weight of wave function of the surface flat band on each layer is shown in Fig. 21(c). It penetrates just three layers into bulk with most of the weight on the surface layer. The surface localization of these flat bands is well resolved for those separated from bulk bands. The nestled flat surface states have small dispersion and their eigen energy distribution in surface BZ is shown in Fig. 21(d), which looks like some vibrational mode of "drumhead". Such "drumhead"-like states are readily to be detected by angle-resolved photoelectron spectroscopy or scanning tunnel microscope.

Recently, there have been many other efforts in studying the NLSM, ${ }^{161-169}$ including the theoretical proposal of $\mathrm{Cu}_{3} \mathrm{PdN}$ to be introduced in the following.

\section{2: Anti-Perovskite $\mathrm{Cu}_{3} \mathrm{PdN}$}

In this section, cubic anti-perovskite $\mathrm{Cu}_{3} \mathrm{PdN}$ is introduced. ${ }^{164}$ It is another example system hosts Node-Line semimetal state protected by the coexistence of TRS and IS in case with band inversion and negligible SOC. However, Pd is heavy element and quite strong SOC might open band gap along the nodal line as discussed in Section 3. 2. Interestingly, in $\mathrm{Cu}_{3} \mathrm{PdN}$, the $\mathrm{C}_{4}$ rotational symmetry will protect the nodal points on the rotation axis 

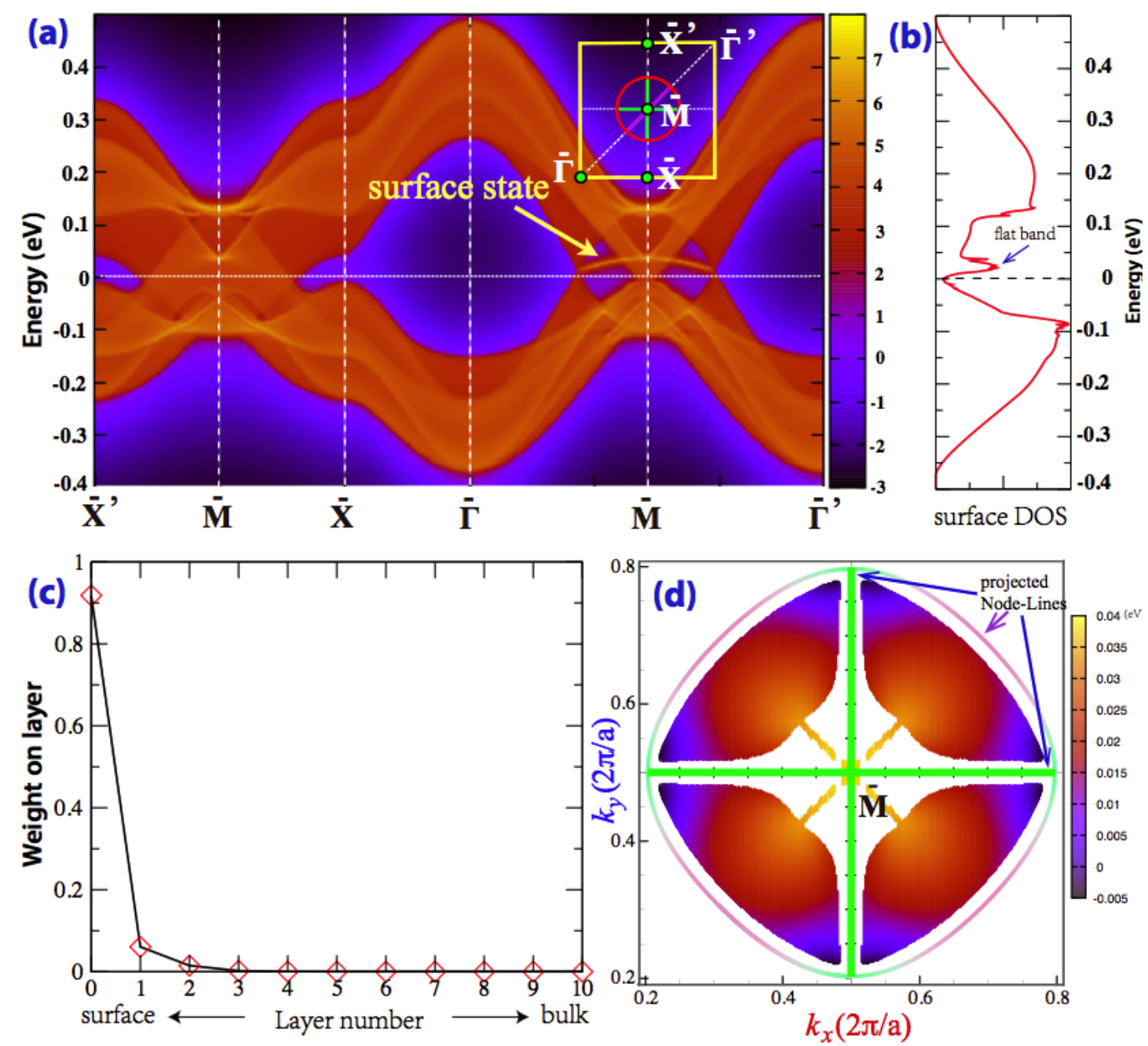

FIG. 21: (Color online) The (001)-surface state. (a) The nearly flat surface band is nestled between two solid Dirac cones, which are the projection of one of the node-line circles as indicated in the inset (red circle). The other two node-line rings are projected as two orthogonal diameters (green line). (b) The surface density of state. (c) The wave function of surface state pointed by the arrow decay rapidly into bulk. (d) The eigen energy distribution of surface flat band nestled inside of projected node-line circle, which looks like a vibration model of "drumhead". The mixing of surface and bulk state leads to discontinuous in this plot.

and leads to DSM state. This is totally different from the situation in TaAs, where SOC opens band gap along the whole nodal ring but results in Weyl nodes off the nodal ring plane. Thus, $\mathrm{Cu}_{3} \mathrm{PdN}$ is a DSM with three pairs of Dirac nodes distinguished from $\mathrm{Na}_{3} \mathrm{Bi}$ and $\mathrm{Cd}_{3} \mathrm{As}_{2}$ with only one pair as shown in Section 2. 1 and 2. 2, respectively. 

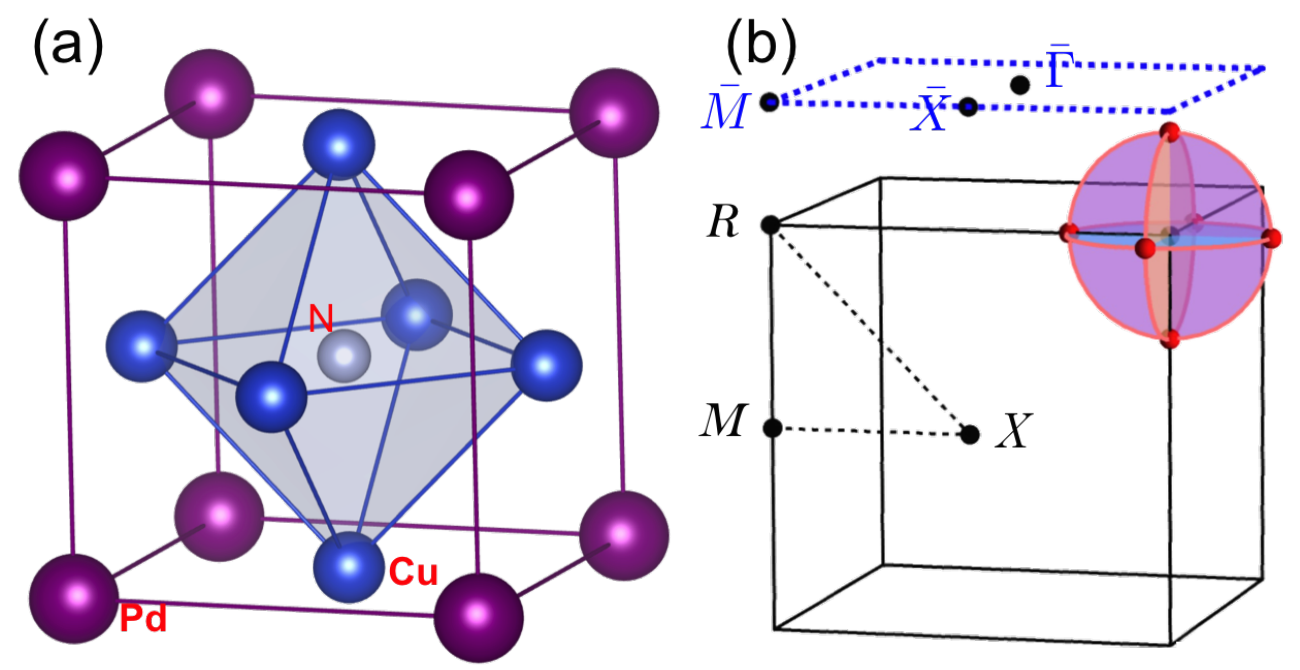

FIG. 22: (Color online) (a) Crystal structure of anti-perovskite $\mathrm{Cu}_{3} \mathrm{PdN}$ with $\mathrm{Pm} \overline{3} \mathrm{~m}$ (No.221) symmetry. Nitrogen atom is at the center of the cube and is surrounded by octahedral $\mathrm{Cu}$ atoms. Pd is located at the corner of the cube. (b) Bulk and projected (001) surface Brillouin zone. The three nodal line rings (orange color) and three pairs of Dirac points (red points) without and with SOC included, respectively, are schematically shown.

Cubic perovskite $\mathrm{Cu}_{3} \mathrm{PdN}$ is shown in Fig. 22 together with its $\mathrm{BZ}$ and projected surface BZ. The band structure of $\mathrm{Cu}_{3} \mathrm{PdN}$ is shown in Fig. 23. The valence and conduction bands are mainly from $\mathrm{Pd}-4 d$ (blue) and $\mathrm{Pd}-5 p$ (red) states, respectively. The fatted bands indicate there is band inversion at $\mathrm{R}$ with $\mathrm{Pd}-5 p$ lower than $\mathrm{Pd}-4 d$ by about $1.5 \mathrm{eV}$. This band inversion is checked by hybrid functional ${ }^{56,57}$ calculation. If SOC is neglected the occupied and unoccupied low energy bands are triply degenerate at $\mathrm{R}$. These states belong to the 3D irreducible representations $\Gamma_{4}^{-}$and $\Gamma_{5}^{+}$of $O_{h}$ group at R point, respectively. The band inversion process in $\mathrm{Cu}_{3} \mathrm{PdN}$ is shown explicitly in Fig. 23(b), where the energy levels of $\Gamma_{5}^{+}$and $\Gamma_{4}^{-}$bands at $\mathrm{R}$ point are calculated under different hydrostatic strains. The band inversion happens at $a=1.11 a_{0}$ ( $a_{0}$ is experimental lattice constant) and the inversion energy increases as further compressing the lattice.

The intriguing point of the $\mathrm{Cu}_{3} \mathrm{PdN}$ band structure without SOC is that the band crossings due to the band inversion form a nodal ring because of the coexistence of TRS and IS as addressed in Section 4. 1. Due to the cubic symmetry, there are three mutually perpendicular nodal rings centering $\mathrm{R}$ as schematically shown in Fig. 22. 

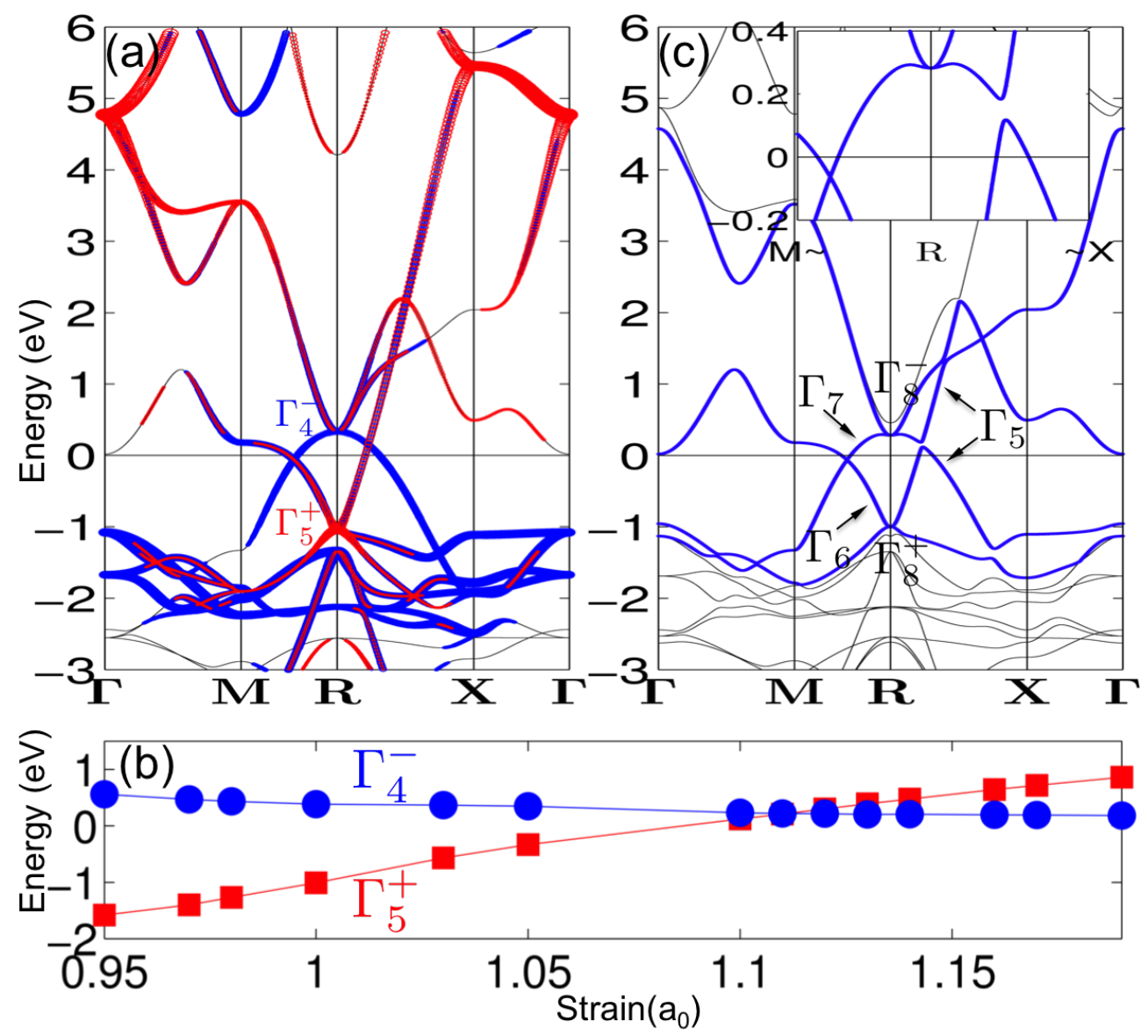

FIG. 23: (Color online) (a) Electronic band structure without SOC, where the component of Pd-5p (4d) orbitals is proportional to the width of the red (blue) curves. Band inversion between $p$ and $d$ orbits happens at R point. (b) Evolution of energy levels of $\Gamma_{5}^{+}$and $\Gamma_{4}^{-}$at

$\mathrm{R}$ under hydrostatic pressure. Band inversion happens when $a<1.11 a_{0}$. (c) Electronic band structure with SOC included. A small gap is opened in the R-X direction while the Dirac point in the R-M direction is stable and protected by crystal symmetry $\mathrm{C}_{4}$ rotation.

When SOC is considered with spin degree of freedom included, the six-fold degenerate states at $\mathrm{R}$ are split into one four-fold and one two-fold degenerate states. Around the Fermi level, the occupied one has $\Gamma_{8}^{+}$symmetry and the unoccupied one has $\Gamma_{8}^{-}$symmetry. Both of them are four-fold degenerate. Along R-X direction, the little group symmetry is lowered to $C_{2 v}$. The four-fold degenerate states at $\mathrm{R}$ are split into two-fold degenerate states. The two bands seem to cross each other have the same $\Gamma_{5}$ symmetry and a gap $\sim 0.062 \mathrm{eV}$ is opened at the intersection as shown in the inset of Fig. 23(b).

However, along R-M direction, the symmetry is characterized by $C_{4 v}$ double group. As 
indicated in Fig. 23(c), the two sets of bands close to Fermi energy belong to $\Gamma_{7}$ and $\Gamma_{6}$ representation, respectively. They are decoupled and the crossing point on $\mathrm{R}-\mathrm{M}$ path is unaffected by SOC.They form a Dirac node near the Fermi energy as shown in Fig. 23(c), which is protected by $C_{4}$ rotational symmetry ${ }^{53,54,170}$. If $C_{4}$ rotational symmetry is broken, the Dirac node will be gapped and the $\mathrm{Z}_{2}$ indices of such gapped system are $(1 ; 111)$, indicating a strong TI. The band structure of $\mathrm{Cu}_{3} \mathrm{PdN}$ is different from that of antiperovskite $\mathrm{Sr}_{3} \mathrm{PbO}^{171}$ and $\mathrm{Ca}_{3} \mathrm{PbO}^{172}$, where the band inversion happens at $\Gamma$ point and the involved bands belong to the same irreducible presentation, which leads to anti-crossing along $\Gamma$-X direction.

The band inversion and the 3D Dirac cones in $\mathrm{Cu}_{3} \mathrm{PdN}$ suggest the presence of topologically nontrivial surface states. The calculated band structures and surface density of states (DOS) on semi-infinite (001) surface are presented in Fig. 24.

Without SOC, the bulk state is the same as $\mathrm{MTC}^{151}$ and there exists surface flat bands nestled inside the projected nodal line ring on the (001) surface, namely the "drumhead" states as shown in Figure 24(a). The peak-like DOS from these nearly flat bands is also clearly shown. The small dispersion of this "drumhead" state comes from the fact that the nodal line ring is not necessarily on the same energy level due to the particle-hole asymmetry $^{61,151,173}$. Such 2D flat bands and nearly infinite DOS are proposed as a route to achieving high-temperature superconductivity ${ }^{159,160,173}$.

In the presence of SOC, each ring is driven into one pair of Dirac nodes. The (001) surface state band structure in Fig. 24(b) clearly shows the gapped bulk state along $\bar{\Gamma}-\bar{M}$ direction and the existence of surface Dirac cone due to topologically nontrivial $\mathrm{Z}_{2}$ indices as seen in $\mathrm{Na}_{3} \mathrm{Bi}^{53}$ and $\mathrm{Cd}_{3} \mathrm{As}_{2} \cdot{ }^{54}$ The bulk band structure along $\mathrm{R}-\mathrm{X}$ and $\mathrm{R}-\mathrm{M}$ overlap each other when projected onto (001) surface along the $\bar{X}-\bar{M}$ path. The bulk Dirac cones are hidden by other bulk bands. Therefore, it is difficult to identify the detailed connection of Fermi arcs in the Fermi surface plotting as shown in Fig. 25, though some eyebrow-like Fermi arcs can be clearly seen around these projected Dirac nodes.

\section{Discussion and Prospect}

Topological semimetals, as a nontrivial extension of topological classification of electronic quantum state from insulator to metal, have been a spotlight in the field of condensed matter

physics in recent years. The theoretical prediction of these topologically nontrivial quantum state has played a crucial role in stimulating this field greatly. An uncompleted survey on the 

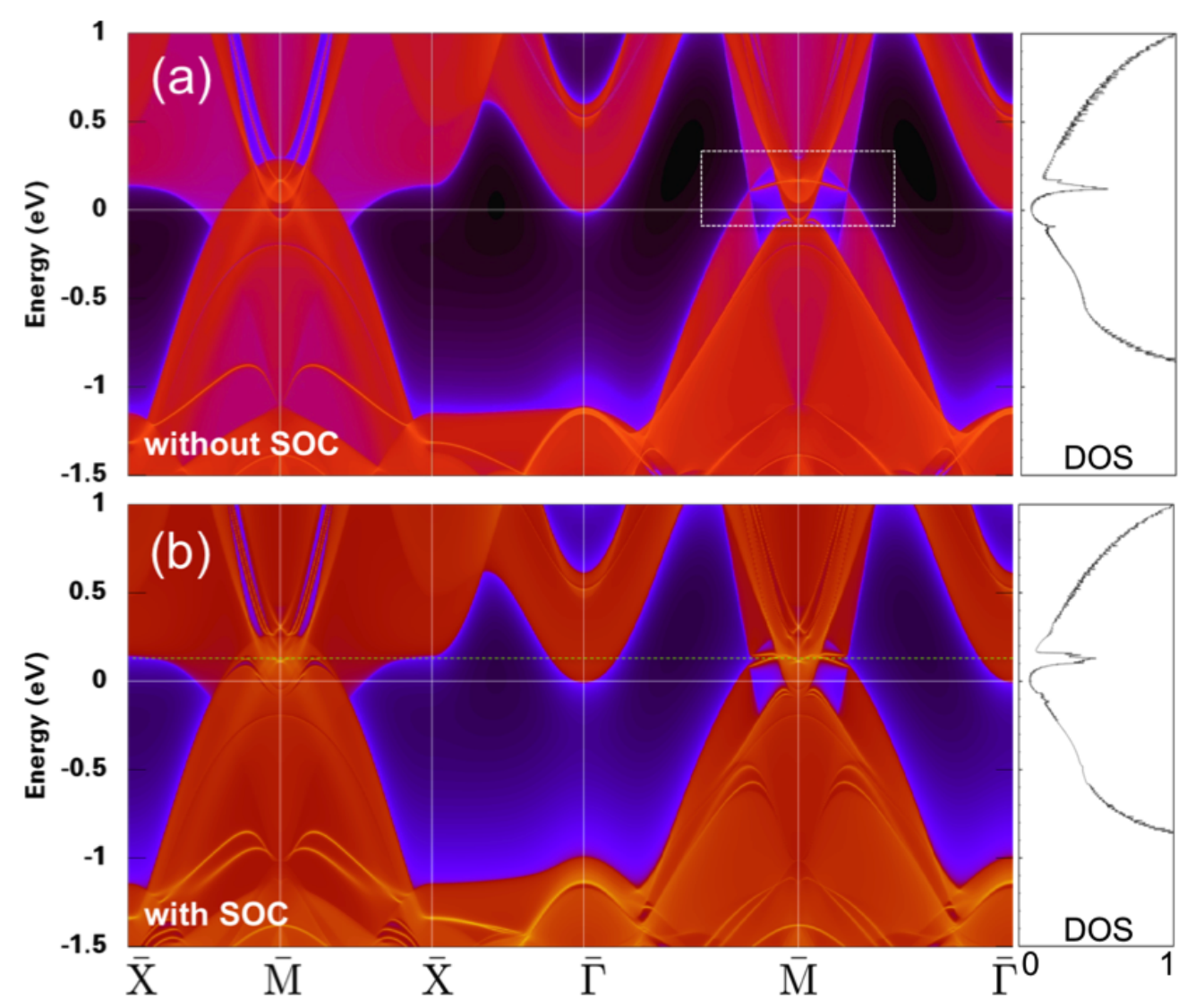

FIG. 24: (Color online) Band structures and DOS for (001) surface (a) without and (b) with SOC . Without SOC, the nearly flat surface bands are clearly shown in the white dashed box around $\bar{M}$ point.

existing theoretical proposals of candidate compounds for each member of TSMs have been listed in Table. II. The DSM classification is following those of Ref. 174. Presently widely studied DSM materials $\mathrm{Na}_{3} \mathrm{Bi}$ and $\mathrm{Cd}_{3} \mathrm{As}_{2}$ are both predicted firstly and then confirmed experimentally. The first WSM of TaAs family is also predicted by theoretical calculation. and recently they are under intensive study. The proposed $\mathrm{FM}$ half-metal states in $\mathrm{HgCr}_{2} \mathrm{Se}_{4}$ has also been confirmed and it is now much close to the final confirmation of its double WSM state. NLSM is the next challenge to be discovered and the predicted candidates are waiting for their experimental verifications.

As mentioned in the introduction, the most interesting phenomena cause by the existence of the Weyl points near the Fermi energy is so called chiral anomaly, where the electrons under parallel magnetic and electric field will be pumped from one Weyl nodes to another one with opposite chirality. In solid states, this pumping process will be eventually balanced 

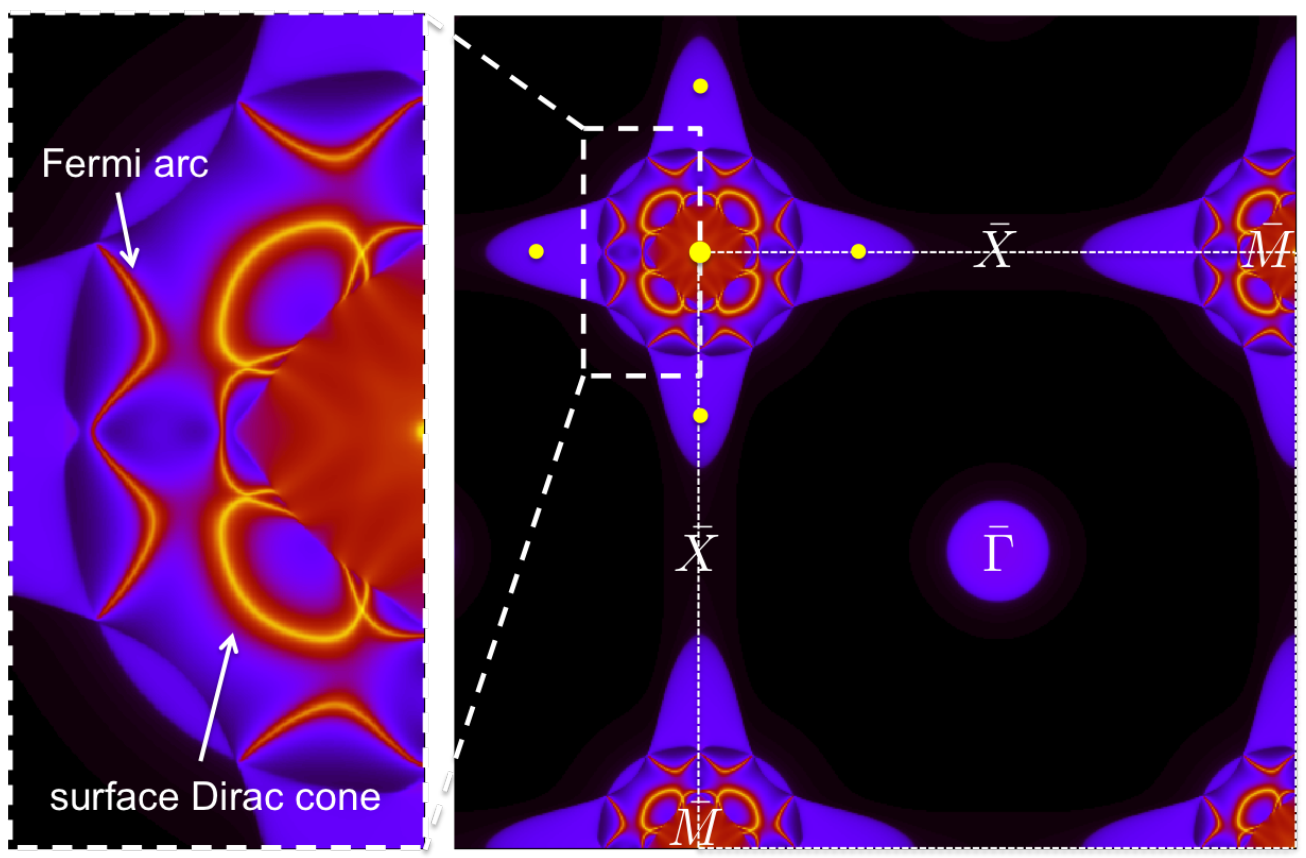

FIG. 25: (Color online) Fermi surface of (001) surface shown in Fig. 24(b) with chemical potential at $0.12 \mathrm{eV}$ (green dashed line in Fig. 24 (b). The yellow dots are projected Dirac points. The bigger one at $\bar{M}$ means there are two Dirac points superposed there.

by the inter-valley scattering terms generated by the impurities leading to a steady state carrying extra chiral current along the direction of the magnetic field with the strength being promotional to the inner product of $\mathrm{E}$ and $\mathrm{B}$ fields. How chiral pumping effect can manifests itself in experimental measurable quantities is one of the key issues in the study of TSM now. Both theoretical analysis and experimental studies seem to suggest that the chiral anomaly will cause universal negative magneto resistance being proportional to $-\mathrm{B}^{2}$ only in the weak magnetic field limit. In the quantum limit under strong magnetic field, the inter Valley scattering rate will depend on field strength as well, which make the behavior of magneto resistance in quantum limit to be related to the detail properties of the impurity potential and non universal. Although the negative magneto resistance under parallel magnetic and electric fields has been experimentally observed in several Weyl, Dirac and even massive Dirac materials, how this can be explained uniquely by chiral anomaly is still far from clear. Other effects that may induced by chiral anomaly, i.e. chiral magnetic effect, nonlocal transport effect and unique optical properties, are still to be observed experimentally.

Through this topical review, the relationship among these TSMs is revealed and summa- 


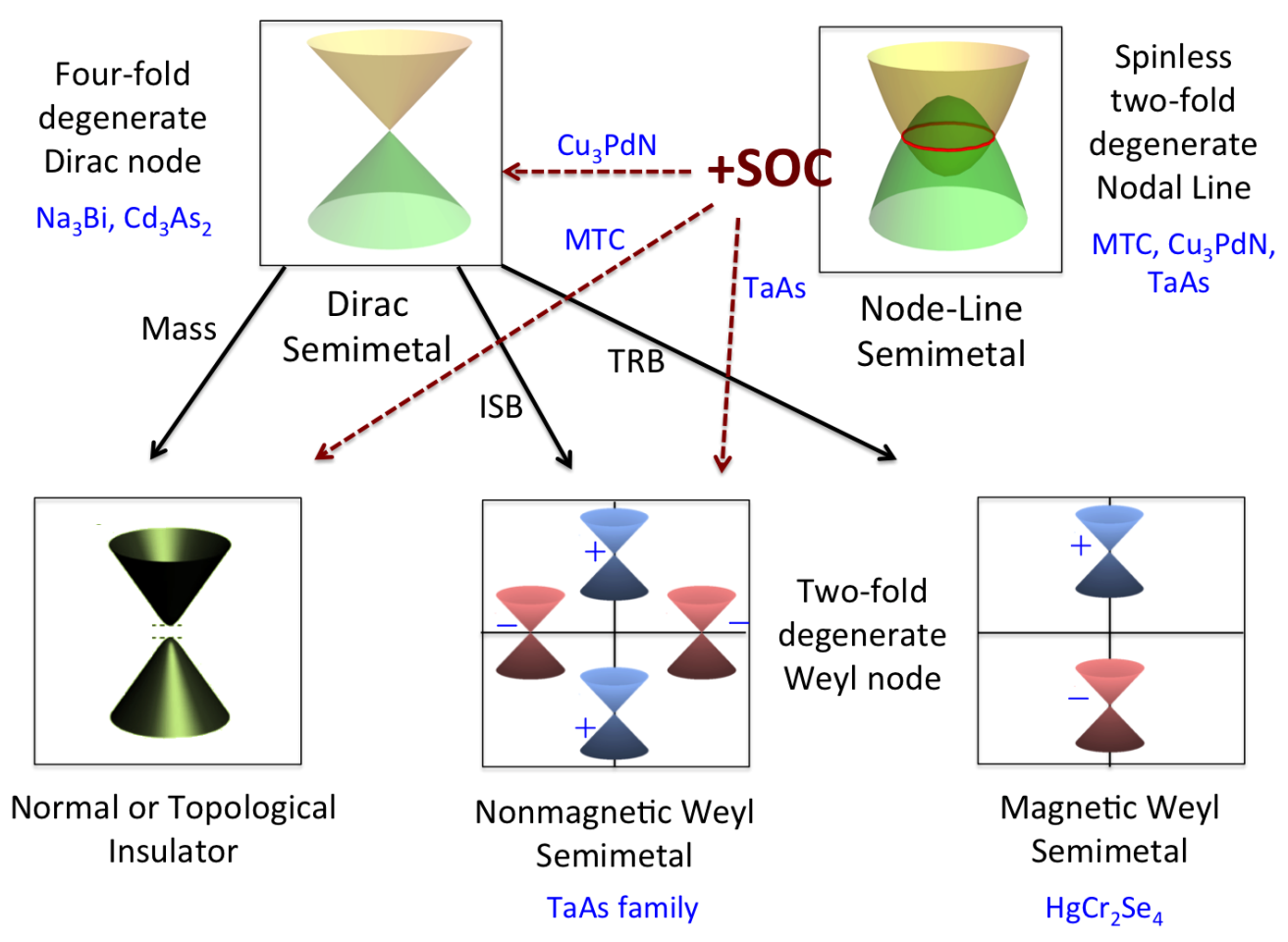

FIG. 26: (Color online) Topological Semimetal (TSM) family and their relationship with each other.

rized in Fig. 26. In the spinless case without including SOC, the coexistence of TRS and IS protects the NLSM state where the band inversion leads to nodal points. Starting from such NLSM, including SOC might lead it to DSM (such as $\mathrm{Cu}_{3} \mathrm{PdN}$ ), TI (such as MTC) and WSM (such as TaAs family). For DSM, introducing different mass term can drive it to normal insulator or TI and breaking either TRS or IS will lead to WSM.

\section{Acknowledgement}

H.M.W., X.D. and Z.F. are supported by the National Science Foundation of China, the 973 program of China (No. 2011CBA00108 and 2013CB921700), and the "Strategic Priority Research Program (B)" of the Chinese Academy of Sciences (No. XDB07020100).

\section{References}

\footnotetext{
* hmweng@iphy.ac.cn

† daix@iphy.ac.cn

‡ zfang@iphy.ac.cn
} 
TABLE II: An uncompleted survey of existing proposals for compounds hosting TSM.

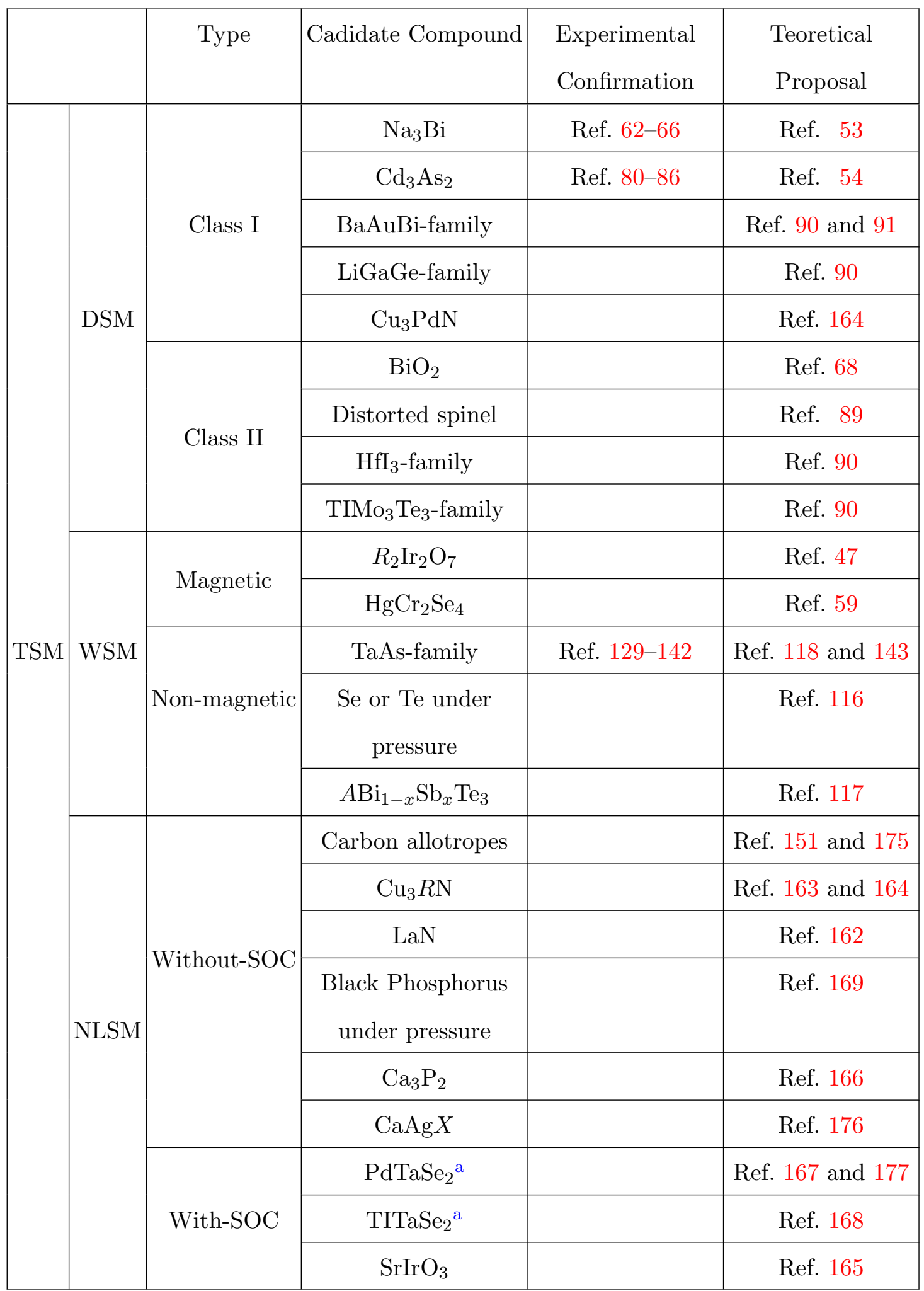

a These candidates have other bulk Fermi surface mixed with proposed Node-Lines. 
1 K. V. Klitzing, G. Dorda, and M. Pepper, "New method for high-accuracy determination of the fine-structure constant based on quantized Hall resistance," Phys. Rev. Lett. 45, 494-497 (1980).

2 D. J. Thouless, M. Kohmoto, M. P. Nightingale, and M. den Nijs, "Quantized Hall conductance in a two-dimensional periodic potential," Phys. Rev. Lett. 49, 405-408 (1982).

3 R. B. Laughlin, "Quantized Hall conductivity in two dimensions," Phys. Rev. B 23, 5632-5633 (1981).

4 F. D. M. Haldane, "Model for a quantum Hall effect without landau levels: Condensed-matter realization of the "Parity anomaly"," Phys. Rev. Lett. 61, 2015-2018 (1988).

5 C. L. Kane and E. J. Mele, " $\mathrm{Z}_{2}$ topological order and the quantum spin Hall effect," Phys. Rev. Lett. 95, 146802 (2005).

6 B. A. Bernevig and S. C. Zhang, "Quantum spin Hall effect," Phys. Rev. Lett. 96, 106802 (2006).

7 M. Z. Hasan and C. L. Kane, "Colloquium: Topological insulators," Rev. Mod. Phys. 82, 3045-3067 (2010).

8 X. L. Qi and S. C. Zhang, "Topological insulators and superconductors," Rev. Mod. Phys. 83, 1057-1110 (2011).

9 C. L. Kane and E. J. Mele, "Quantum spin Hall effect in graphene," Phys. Rev. Lett. 95, 226801 (2005).

10 Yugui Yao, Fei Ye, Xiao-Liang Qi, Shou-Cheng Zhang, and Zhong Fang, "Spin-orbit gap of graphene: First-principles calculations," Phys. Rev. B 75, 041401 (2007).

11 B. A. Bernevig, T. L. Hughes, and S. C. Zhang, "Quantum spin Hall effect and topological phase transition in HgTe quantum wells," Science 314, 1757-1761 (2006).

12 M. König, S. Wiedmann, C. Brüne, A. Roth, H. Buhmann, L. W. Molenkamp, X. L. Qi, and S. C. Zhang, "Quantum spin Hall insulator state in HgTe quantum wells," Science 318, 766-770 (2007).

13 H. J. Zhang, C. X. Liu, X. L. Qi, X. Dai, Z. Fang, and S. C. Zhang, "Topological insulators in $\mathrm{Bi}_{2} \mathrm{Se}_{3}, \mathrm{Bi}_{2} \mathrm{Te}_{3}$ and $\mathrm{Sb}_{2} \mathrm{Te}_{3}$ with a single dirac cone on the surface," Nature Physics 5, 438-442 (2009).

14 Y. Xia, D. Qian, D. Hsieh, L. Wray, A. Pal, H. Lin, A. Bansil, D. Grauer, Y. S. Hor, R. J. Cava, and M. Z. Hasan, "Observation of a large-gap topological-insulator class with a single 
Dirac cone on the surface," Nature Physics 5, 398-402 (2009).

15 Y. L. Chen, J. G. Analytis, J.-H. Chu, Z. K. Liu, S.-K. Mo, X. L. Qi, H. J. Zhang, D. H. Lu, X. Dai, Z. Fang, S. C. Zhang, I. R. Fisher, Z. Hussain, and Z.-X. Shen, "Experimental realization of a three-dimensional topological insulator, $\mathrm{Bi}_{2} \mathrm{Te}_{3}$," Science 325, 178-181 (2009). Yong Xu, Zhongxue Gan, and Shou-Cheng Zhang, "Enhanced thermoelectric performance and anomalous seebeck effects in topological insulators," Phys. Rev. Lett. 112, 226801 (2014).

17 Hermann Osterhage, Johannes Gooth, Bacel Hamdou, Paul Gwozdz, Robert Zierold, and Kornelius Nielsch, "Thermoelectric properties of topological insulator $\mathrm{Bi}_{2} \mathrm{Te}_{3}, \mathrm{Sb}_{2} \mathrm{Te}_{3}$, and $\mathrm{Bi}_{2} \mathrm{Se}_{3}$ thin film quantum wells," Applied Physics Letters 105, 123117 (2014).

18 L. Fu and C. L. Kane, "Superconducting proximity effect and Majorana fermions at the surface of a topological insulator," Phys. Rev. Lett. 100, 096407 (2008).

19 X. L. Qi, T. L. Hughes, and S. C. Zhang, "Chiral topological superconductor from the quantum Hall state," Phys. Rev. B 82, 184516 (2010).

20 J. L. Zhang, S. J. Zhang, H. M. Weng, W. Zhang, L. X. Yang, Q. Q. Liu, S. M. Feng, X. C. Wang, R. C. Yu, and L. Z. Cao, "Pressure-induced superconductivity in topological parent compound $\mathrm{Bi}_{2} \mathrm{Te}_{3}, "$ Proc. Nat. Asso. Soc. 108, 24-28 (2011).

21 M. Onoda and N. Nagaosa, "Quantized anomalous Hall effect in two-dimensional ferromagnets: Quantum Hall effect in metals," Phys. Rev. Lett. 90, 206601 (2003).

22 Chao-Xing Liu, Xiao-Liang Qi, Xi Dai, Zhong Fang, and Shou-Cheng Zhang, "Quantum Anomalous Hall Effect in $\mathrm{Hg}_{1-y} \mathrm{Mn}_{y}$ Te Quantum Wells," Phys. Rev. Lett. 101, 146802 (2008).

23 Hongming Weng, Rui Yu, Xiao Hu, Xi Dai, and Zhong Fang, "Quantum anomalous hall effect and related topological electronic states," Adv. Phys. 64, 227-282 (2015).

24 R. Yu, W. Zhang, H. J. Zhang, S. C. Zhang, X. Dai, and Z. Fang, "Quantized anomalous Hall effect in magnetic topological insulators," Science 329, 61-64 (2010).

25 C. Z. Chang, J. S. Zhang, X. Feng, J. Shen, Z. C. Zhang, M. H. Guo, K. Li, Y. B. Ou, P. Wei, L. L. Wang, Z. Q. Ji, Y. Feng, S. H. Ji, X. Chen, J. F. Jia, X. Dai, Z. Fang, S. C. Zhang, K. He, Y. Y. Wang, L. Lu, X. C. Ma, and Q. K. Xue, "Experimental observation of the quantum anomalous Hall effect in a magnetic topological insulator," Science 340, 167-170 (2013).

26 H. Weyl, "Elektron und Gravitation. I,” Zeitschrift für Physik 56, 330-352 (1929).

27 Z. Fang, N. Nagaosa, K. S. Takahashi, A. Asamitsu, R. Mathieu, T. Ogasawara, H. Yamada, M. Kawasaki, Y. Tokura, and K. Terakura, "The anomalous Hall effect and magnetic 
monopoles in momentum space," Science 302, 92-95 (2003).

28 H. B. Nielsen and M. Ninomiya, "Absence of neutrinos on a lattice: (I). Proof by homotopy theory," Nuclear Physics B 185, 20-40 (1981).

29 H. B. Nielsen and M. Ninomiya, "Absence of neutrinos on a lattice: (II). Intuitive topological proof," Nuclear Physics B 193, 173-194 (1981).

30 L. Balents, "Weyl electrons kiss," Physics 4, 36 (2011).

31 H. B. Nielsen and M. Ninomiya, "The Adler-Bell-Jackiw anomaly and Weyl fermions in a crystal," Physics Letters B 130, 389-396 (1983).

32 D. T. Son and B. Z. Spivak, "Chiral anomaly and classical negative magnetoresistance of Weyl metals," Phys. Rev. B 88, 104412 (2013).

33 Ki-Seok Kim, Heon-Jung Kim, and M. Sasaki, "Boltzmann equation approach to anomalous transport in a weyl metal," Phys. Rev. B 89, 195137 (2014).

34 Pavan Hosur, S. A. Parameswaran, and Ashvin Vishwanath, "Charge transport in weyl semimetals," Phys. Rev. Lett. 108, 046602 (2012).

35 Phillip E. C. Ashby and J. P. Carbotte, "Chiral anomaly and optical absorption in weyl semimetals," Phys. Rev. B 89, 245121 (2014).

36 M. R. Norman, "Vector Optical Activity in the Weyl Semimetal TaAs," arXiv:1510.01802 (2015).

37 R. Yu, H. Weng, Z. Fang, H. Ding, and X. Dai, "Determine the chirality of Weyl fermions from the circular dichroism spectra of time-dependent ARPES,” arXiv:1512.04055 (2015).

38 C.-K. Chan, P. A. Lee, K. S. Burch, J. H. Han, and Y. Ran, "When chiral photons meet chiral fermions - Photoinduced anomalous Hall effects in Weyl semimetals," arXiv:1509.05400 (2015).

39 Pavan Hosur and Xiaoliang Qi, "Recent developments in transport phenomena in weyl semimetals," Comptes Rendus Physique 14, 857-870 (2013).

40 Qiang Li, Dmitri E. Kharzeev, Cheng Zhang, Yuan Huang, I. Pletikosic, A. V. Fedorov, R. D. Zhong, J. A. Schneeloch, G. D. Gu, and T. Valla, "Observation of the chiral magnetic effect in $\mathrm{ZrTe}_{5}, "$ arXiv:1412.6543 (2014).

41 S. A. Parameswaran, T. Grover, D. A. Abanin, D. A. Pesin, and A. Vishwanath, "Probing the chiral anomaly with nonlocal transport in three-dimensional topological semimetals," Phys. Rev. X 4, 031035 (2014). 
42 Chao-Xing Liu, Peng Ye, and Xiao-Liang Qi, "Chiral gauge field and axial anomaly in a weyl semimetal," Phys. Rev. B 87, 235306 (2013).

43 M. V. Berry, "Quantal phase factors accompanying adiabatic changes," Proc. R. Soc. Lond. A 392, 45-57 (1984).

44 Hongming Weng, Xi Dai, and Zhong Fang, "Exploration and prediction of topological electronic materials based on first-principles calculations," MRS Bulletin 39, 849-858 (2014).

45 L. Fu, "Topological Crystalline Insulators," Phys. Rev. Lett. 106, 106802 (2011).

46 T. H. Hsieh, H. Lin, J. W. Liu, W. H. Duan, A. Bansi, and L. Fu, "Topological crystalline insulators in the SnTe material class," Nature Communications 3, 982 (2012).

47 X. G. Wan, A. M. Turner, A. Vishwanath, and S. Y. Savrasov, "Topological semimetal and fermi-arc surface states in the electronic structure of pyrochlore iridates," Phys. Rev. B 83, 205101 (2011).

48 P. A. M. Dirac, "The quantum theory of the electron," Proc. R. Soc. Lond. A 117, 610-624 (1928).

49 Anthony J. Leggett, "A theoretical description of the new phases of liquid ${ }^{3} \mathrm{He}, "$ Rev. Mod. Phys. 47, 331-414 (1975).

50 G. E. Volovik, The Universe in a Helium Droplet (Oxford, 2009).

51 S. Murakami, "Phase transition between the quantum spin Hall and insulator phases in 3d: emergence of a topological gapless phase," New J. Phys. 9, 356 (2007).

52 Shuichi Murakami, "Gap closing and universal phase diagrams in topological insulators," Physica E: Low-dimensional Systems and Nanostructures 43, 748-754 (2011).

53 Z. J. Wang, Y. Sun, X. Q. Chen, C. Franchini, G. Xu, H. M. Weng, X. Dai, and Z. Fang, "Dirac semimetal and topological phase transitions in $A_{3} \mathrm{Bi}(A=\mathrm{Na}, \mathrm{K}, \mathrm{Rb})$," Phys. Rev. B 85, 195320 (2012).

54 Z. J. Wang, H. M. Weng, Q. S. Wu, X. Dai, and Z. Fang, "Three-dimensional Dirac semimetal and quantum transport in $\mathrm{Cd}_{3} \mathrm{As}_{2}$," Phys. Rev. B 88, 125427 (2013).

55 A. R. H. F. Ettema and R. A. de Groot, "Electronic structure of $\mathrm{Na}_{3} \mathrm{Sb}$ and $\mathrm{Na}_{2} \mathrm{KSb}$," Phys. Rev. B 61, 10035-10039 (2000).

56 Jochen Heyd, Gustavo E. Scuseria, and Matthias Ernzerhof, "Hybrid functionals based on a screened coulomb potential," The Journal of Chemical Physics 118, 8207-8215 (2003).

57 Jochen Heyd, Gustavo E. Scuseria, and Matthias Ernzerhof, "Erratum: hybrid function- 
als based on a screened coulomb potential [j. chem. phys.118, 8207 (2003)]," The Journal of Chemical Physics 124, 219906 (2006).

58 W. Zhang, R. Yu, H. J. Zhang, X. Dai, and Z. Fang, "First-principles studies of the threedimensional strong topological insulators $\mathrm{Bi}_{2} \mathrm{Te}_{3}, \mathrm{Bi}_{2} \mathrm{Se}_{3}$ and $\mathrm{Sb}_{2} \mathrm{Te}_{3}$," New J. Phys. 12, 065013 (2010).

59 G. Xu, H. M. Weng, Z. J. Wang, X. Dai, and Z. Fang, "Chern Semimetal and the Quantized Anomalous Hall Effect in $\mathrm{HgCr}_{2} \mathrm{Se}_{4}$," Phys. Rev. Lett. 107, 186806 (2011).

60 A. A. Burkov and L. Balents, "Weyl Semimetal in a Topological Insulator Multilayer," Phys. Rev. Lett. 107, 127205 (2011).

61 A. A. Burkov, M. D. Hook, and L. Balents, "Topological nodal semimetals," Phys. Rev. B 84, 235126 (2011).

62 Z. K. Liu, B. Zhou, Y. Zhang, Z. J. Wang, H. M. Weng, D. Prabhakaran, S. K. Mo, Z. X. Shen, Z. Fang, X. Dai, Z. Hussain, and Y. L. Chen, "Discovery of a Three-Dimensional Topological Dirac Semimetal, Na 3 Bi," Science 343, 864-867 (2014).

63 Su-Yang Xu, Chang Liu, S. K. Kushwaha, T. R. Chang, J. W. Krizan, R. Sankar, C. M. Polley, J. Adell, T. Balasubramanian, K. Miyamoto, N. Alidoust, Guang Bian, M. Neupane, I. Belopolski, H. T. Jeng, C. Y. Huang, W. F. Tsai, H. Lin, F. C. Chou, T. Okuda, A. Bansil, R. J. Cava, and M. Z. Hasan, "Observation of a bulk 3D Dirac multiplet, Lifshitz transition, and nestled spin states in $\mathrm{Na}_{3} \mathrm{Bi}, "$ arXiv:1312.7624 (2013).

64 S.-Y. Xu, C. Liu, S. K. Kushwaha, R. Sankar, J. W. Krizan, I. Belopolski, M. Neupan, e, G. Bian, N. Alidoust, T.-R. Chang, H.-T. Jeng, C.-Y. Huang, W.-F. Tsai, H. Lin, P. P. Shibayev, F. Chou, R. J. Cava, and M. Z. Hasan, "Observation of Fermi arc surface states in a topological metal," Science 347, 294-298 (2015).

65 J. Xiong, S. K. Kushwaha, T. Liang, J. W. Krizan, W. Wang, R. J. Cava, and N. P. Ong, "Signature of the chiral anomaly in a Dirac semimetal: a current plume steered by a magnetic field," arXiv:1503.08179 (2015).

66 Jun Xiong, Satya K. Kushwaha, Tian Liang, Jason W. Krizan, Max Hirschberger, Wudi Wang, R. J. Cava, and N. P. Ong, "Evidence for the chiral anomaly in the dirac semimetal na3bi," Science 350, 413-416 (2015).

67 Anton Burkov, "Chiral anomaly without relativity," Science 350, 378-379 (2015).

68 S. M. Young, S. Zaheer, J. C. Y. Teo, C. L. Kane, E. J. Mele, and A. M. Rappe, "Dirac 
semimetal in three dimensions," Phys. Rev. Lett. 108, 140405 (2012).

69 W. Zdanowicz and L. Zdanowicz, "Semiconducting Compounds of the AII BV Group," Annu. Rev. Mater. Sci. 5, 301-328 (1975).

70 P. J. LIN-CHUNG, "Energy-band structures of cd $_{3} a_{2}$ and $z_{3} a_{2}$," Phys. Rev. 188, 1272-1280 (1969).

71 K. Sierański, J. Szatkowski, and J. Misiewicz, "Semiempirical tight-binding band structure of $\mathrm{II}_{3} \mathrm{~V}_{2}$ semiconductors: $\mathrm{Cd}_{3} \mathrm{P}_{2}, \mathrm{Zn}_{3} \mathrm{P}_{2}, \mathrm{Cd}_{3} \mathrm{As}_{2}$, and $\mathrm{Zn}_{3} \mathrm{As}_{2}$," Phys. Rev. B 50, 7331-7337 (1994).

72 G. A. Steigmann and J. Goodyear, "The crystal structure of $\mathrm{Cd}_{3} \mathrm{As}_{2}$," Acta Crystallographica Section B 24, 1062-1067 (1968).

73 Mazhar N. Ali, Quinn Gibson, Sangjun Jeon, Brian B. Zhou, Ali Yazdani, and R. J. Cava, "The Crystal and Electronic Structures of $\mathrm{Cd}_{3} \mathrm{As}_{2}$, the Three-Dimensional Electronic Analogue of Graphene," Inorganic Chemistry 53, 4062-4067 (2014).

74 M. J. Aubin, L. G. Caron, and J. P. Jay-Gerin, "Band structure of cadmium arsenide at room temperature," Phys. Rev. B 15, 3872-3878 (1977).

75 L. G. Caron, J. P. Jay-Gerin, and M. J. Aubin, "Energy-band structure of $\operatorname{cd}_{3}$ as 2 at low temperatures and the dependence of the direct gap on temperature and pressure," Phys. Rev. B 15, 3879-3887 (1977).

76 B. Dowgiao-Plenkiewicz and P. Plenkiewicz, "Inverted band structure of $\mathrm{Cd}_{3} \mathrm{As}_{2}$," Physica Status Solidi (b) 94, K57-K60 (1979).

77 G. Chuiko, N. Don, O. Dvornik, V. Ivchenko, and Sergeyev A., "Simple inverted band structure model for cadmium arsenide $\left(\mathrm{Cd}_{3} \mathrm{As}_{2}\right)$," Moldavian Journal of the Physical Sciences 2, 88 (2003).

78 B. Plenkiewicz, P.R. Wallace, and P. Plenkiewicz, "The role of vacancies in the band structure of $\mathrm{Cd}_{3} \mathrm{As}_{2}, "$ Solid State Communications 50, 681-684 (1984).

79 Wei Zhang, Rui Yu, Wanxiang Feng, Yugui Yao, Hongming Weng, Xi Dai, and Zhong Fang, "Topological aspect and quantum magnetoresistance of $\beta-\mathrm{ag}_{2}$ Te," Phys. Rev. Lett. 106, 156808 (2011).

80 Z. K. Liu, J. Jiang, B. Zhou, Z. J. Wang, Y. Zhang, H. M. Weng, D. Prabhakaran, S. K. Mo, H. Peng, P. Dudin, T. Kim, M. Hoesch, Z. Fang, X. Dai, Z. X. Shen, D. L. Feng, Z. Hussain, and Y. L. Chen, "A stable three-dimensional topological Dirac semimetal $\mathrm{Cd}_{3} \mathrm{As}_{2}$," Nature 
Materials 13, 677-681 (2014).

81 Sergey Borisenko, Quinn Gibson, Danil Evtushinsky, Volodymyr Zabolotnyy, Bernd Büchner, and Robert J. Cava, "Experimental realization of a three-dimensional dirac semimetal," Phys. Rev. Lett. 113, 027603 (2014).

82 H. M. Yi, Z. J. Wang, C. Y. Chen, Y. G. Shi, Y. Feng, A. J. Liang, Z. J. Xie, S. L. He, J. F. He, . Y. Peng, X. Liu, Y. Liu, L. Zhao, G. D. Liu, X. L. Dong, J. Zhang, M. Nakatake, M. Arita, K. Shimada, H. Namatame, M. Taniguchi, Z. Y. Xu, C. T. Chen, X. Dai, Z. Fang, and X. J. Zhou, "Evidence of Topological Surface State in Three-Dimensional Dirac Semimetal $\mathrm{Cd}_{3} \mathrm{As}_{2}$," Scientific Reports 4, 6106 (2014).

83 M. Neupane, S. Y. Xu, R. Sankar, N. Alidoust, G. Bian, C. Liu, I. Belopolski, T. R. Chang, H. T. Jeng, H. Lin, A. Bansil, F. C. Chou, and M. Z. Hasan, "Observation of a threedimensional topological Dirac semimetal phase in high-mobility $\mathrm{Cd}_{3} \mathrm{As}_{2}$," Nature Communications 5, 3786 (2014).

84 S. J. Jeon, B. B. Zhou, A. Gyenis, B. E. Feldman, I. Kimchi, A. C. Potter, Q. D. Gibson, R. J. Cava, A. Vishwanath, and A. Yazdani, "Landau quantization and quasiparticle interference in the three-dimensional dirac semimetal $\mathrm{Cd}_{3} \mathrm{As}_{2}$," Nature Materials 13, 851-856 (2014).

85 L. P. He, X. C. Hong, J. K. Dong, J. Pan, Z. Zhang, J. Zhang, and S. Y. Li, "Quantum Transport Evidence for the Three-Dimensional Dirac Semimetal Phase in $\mathrm{Cd}_{3} \mathrm{As}_{2}$," Phys. Rev. Lett. 113, 246402 (2014).

86 Tian Liang, Quinn Gibson, Mazhar N Ali, Minhao Liu, R J Cava, and N P Ong, "Ultrahigh mobility and giant magnetoresistance in the Dirac semimetal $\mathrm{Cd}_{3} \mathrm{As}_{2}$," Nature materials 14, 280-284 (2015).

87 Junya Feng, Yuan Pang, Desheng Wu, Zhijun Wang, Hongming Weng, Jianqi Li, Xi Dai, Zhong Fang, Youguo Shi, and Li Lu, "Large linear magnetoresistance in Dirac semimetal $\mathrm{Cd}_{3} \mathrm{As}_{2}$ with Fermi surfaces close to the Dirac points," Phys. Rev. B 92, 081306 (2015).

88 Bohm-Jung Yang, Takahiro Morimoto, and Akira Furusaki, "Topological charges of threedimensional dirac semimetals with rotation symmetry," Phys. Rev. B 92, 165120 (2015).

89 Julia A. Steinberg, Steve M. Young, Saad Zaheer, C. L. Kane, E. J. Mele, and Andrew M. Rappe, "Bulk dirac points in distorted spinels," Phys. Rev. Lett. 112, 036403 (2014).

90 Q. D. Gibson, L. M. Schoop, L. Muechler, L. S. Xie, M. Hirschberger, N. P. Ong, R. Car, and R. J. Cava, "Three-dimensional dirac semimetals: Design principles and predictions of new 
materials," Phys. Rev. B 91, 205128 (2015).

91 Y. Du, B. Wan, D. Wang, L. Sheng, C. G. Duan, and Wan X., "Dirac and Weyl Semimetal in XYBi (X=Ba, Eu; Y=Cu, Ag and Au)," Scientific Reports 5, 14423 (2015).

92 H. J. Zhang, C. X. Liu, X. L. Qi, X. Y. Deng, X. Dai, S. C. Zhang, and Z. Fang, "Electronic structures and surface states of the topological insulator $\mathrm{Bi}_{1-x} \mathrm{Sb}_{x}$, , Phys. Rev. B 80, 085307 (2009).

93 Heon-Jung Kim, Ki-Seok Kim, J.-F. Wang, M. Sasaki, N. Satoh, A. Ohnishi, M. Kitaura, M. Yang, and L. Li, "Dirac versus weyl fermions in topological insulators: Adler-bell-jackiw anomaly in transport phenomena," Phys. Rev. Lett. 111, 246603 (2013).

94 H. M. Weng, X. Dai, and Z. Fang, "Transition-metal pentatelluride $\mathrm{ZrTe}_{5}$ and $\mathrm{HfTe}_{5}$ : A paradigm for large-gap quantum spin Hall insulators," Phys. Rev. X 4, 011002 (2014).

95 R. Y. Chen, Z. G. Chen, X.-Y. Song, J. A. Schneeloch, G. D. Gu, F. Wang, and N. L. Wang, "Magnetoinfrared spectroscopy of landau levels and zeeman splitting of three-dimensional massless dirac fermions in zrte 5 ," Phys. Rev. Lett. 115, 176404 (2015).

96 R. Y. Chen, S. J. Zhang, J. A. Schneeloch, C. Zhang, Q. Li, G. D. Gu, and N. L. Wang, "Optical spectroscopy study of the three-dimensional Dirac semimetal ZrTe, , Phys. Rev. B 92, 075107 (2015).

97 Chen Fang, Matthew J. Gilbert, Xi Dai, and B. Andrei Bernevig, "Multi-weyl topological semimetals stabilized by point group symmetry," Phys. Rev. Lett. 108, 266802 (2012).

98 Alexey A Soluyanov, Dominik Gresch, Zhijun Wang, QuanSheng Wu, Matthias Troyer, Xi Dai, and B Andrei Bernevig, "Type-II Weyl semimetals," Nature 527, 495-498 (2015).

99 Mazhar N Ali, Jun Xiong, Steven Flynn, Jing Tao, Quinn D Gibson, Leslie M Schoop, Tian Liang, Neel Haldolaarachchige, Max Hirschberger, N P Ong, and R J Cava, "Large, nonsaturating magnetoresistance in $\mathrm{WTe}_{2}, "$ Nature 514, 205-208 (2014).

100 P. Wojtowicz, "Semiconducting ferromagnetic spinels," IEEE Transactions on Magnetics 5, 840-848 (1969).

101 N.I. Solin, V.V. Ustinov, and S.V. Naumov, "Colossal magnetoresistance of the inhomogeneous ferromagnetic semiconductor $\mathrm{HgCr}_{2} \mathrm{Se}_{4}$," Physics of the Solid State 50, 901-908 (2008).

102 N.I. Solin and N.M. Chebotaev, "Magnetoresistance and Hall effect of the magnetic semiconductor $\mathrm{HgCr}_{2} \mathrm{Se}_{4}$ in strong magnetic fields," Physics of the Solid State 39, 754-758 (1997).

103 Toshihiro Arai, Moriaki Wakaki, Seinosuke Onari, Keiei Kudo, Takehiko Satoh, and Tachiro 
Tsushima, "Magnetoabsorption in Single-Crystal $\mathrm{HgCr}_{2} \mathrm{Se}_{4}$," Journal of the Physical Society of Japan 34, 68-73 (1973).

104 P. Baltzer, H. Lehmann, and M. Robbins, "Insulating Ferromagnetic Spinels," Phys. Rev. Lett. 15, 493-495 (1965).

105 P. Baltzer, P. Wojtowicz, M. Robbins, and E. Lopatin, "Exchange interactions in ferromagnetic chromium chalcogenide spinels," Physical Review 151, 367-377 (1966).

106 H.W. Lehmann and F.P. Emmenegger, "Crystal growth, semiconducting and optical properties of ferromagnetic $\mathrm{HgCr}_{2} \mathrm{Se}_{4}$," Solid State Communications 7, 965-968 (1969).

107 A. Selmi, A. Mauger, and M. Heritier, "Magnetic semiconductors controlled by intra-atomic coulomb correlations: The example of $\mathrm{HgCr}_{2} \mathrm{Se}_{4}$," Journal of Magnetism and Magnetic Materials 66, 295-316 (1987).

108 C. Y. Moon and S. H. Wei, "Band gap of Hg chalcogenides: Symmetry-reduction-induced bandgap opening of materials with inverted band structures," Phys. Rev. B 74, 045205 (2006).

109 A. Delin, "First-principles calculations of the II-VI semiconductor $\beta$-HgS: Metal or semiconductor," Phys. Rev. B 65, 153205 (2002).

110 C. Fennie and K. Rabe, "Polar phonons and intrinsic dielectric response of the ferromagnetic insulating spinel $\mathrm{CdCr}_{2} \mathrm{~S}_{4}$ from first principles," Phys. Rev. B 72, 214123 (2005).

111 A Yaresko, "Electronic band structure and exchange coupling constants in $A \mathrm{Cr}_{2} \mathrm{X}_{4}$ spinels ( $A=\mathrm{Zn}, \mathrm{Cd}, \mathrm{Hg} ; \mathrm{X}=\mathrm{O}, \mathrm{S}, \mathrm{Se})$," Phys. Rev. B 77, 115106 (2008).

112 M. Onoda and N. Nagaosa, "Topological nature of anomalous Hall effect in ferromagnets," J. Phys. Soc. J. 71, 19-22 (2002).

113 Tong Guan, Chaojing Lin, Chongli Yang, Youguo Shi, Cong Ren, Yongqing Li, Hongming Weng, Xi Dai, Zhong Fang, Shishen Yan, and Peng Xiong, "Evidence for Half-Metallicity in n-type $\mathrm{HgCr}_{2} \mathrm{Se}_{4}, "$ Phys. Rev. Lett. 115, 087002 (2015).

114 Daniel Bulmash, Chao-Xing Liu, and Xiao-Liang Qi, "Prediction of a Weyl semimetal in $\mathrm{Hg}_{1-x-y} \mathrm{Cd}_{x} \mathrm{Mn}_{y}$ Te," Phys. Rev. B 89, 081106 (2014).

115 A. A. Zyuzin, S. Wu, and A. A. Burkov, "Weyl semimetal with broken time reversal and inversion symmetries," Phys. Rev. B 85, 165110 (2012).

116 Motoaki Hirayama, Ryo Okugawa, Shoji Ishibashi, Shuichi Murakami, and Takashi Miyake, "Weyl node and spin texture in trigonal tellurium and selenium," Phys. Rev. Lett. 114, 206401 (2015). 
117 J. P. Liu and D. Vanderbilt, "Weyl semimetals from noncentrosymmetric topological insulators," Phys. Rev. B 90, 155316 (2014).

118 Hongming Weng, Chen Fang, Zhong Fang, B. A. Bernevig, and X. Dai, "Weyl semimetal phase in noncentrosymmetric transition-metal monophosphides," Phys. Rev. X 5, 011029 (2015).

119 L. Fu, C. L. Kane, and E. J. Mele, "Topological insulators in three dimensions," Phys. Rev. Lett. 98, 106803 (2007).

120 A. M. Turner, Y. Zhang, R. S. K. Mong, and A. Vishwanath, "Quantized response and topology of magnetic insulators with inversion symmetry," Phys. Rev. B 85, 165120 (2012).

121 Taylor L. Hughes, Emil Prodan, and B. Andrei Bernevig, "Inversion-symmetric topological insulators," Physical Review B 83, 245132 (2011).

122 Hongming Weng, J. Z. Zhao, Z. J. Wang, Z. Fang, and X. Dai, "Topological crystalline Kondo insulator in mixed valence ytterbium borides," Phys. Rev. Lett. 112, 016403 (2014).

123 L. Fu and C. L. Kane, "Time reversal polarization and a $Z_{2}$ adiabatic spin pump," Phys. Rev. B 74, 195312 (2006).

124 L. Fu and C. L. Kane, "Topological insulators with inversion symmetry," Phys. Rev. B 76, 045302 (2007).

125 S. Furuseth, K. Selte, and A. Kjekshus, "On the arsenides and antimonides of tantalum," Acta Chem. Scand. 19, 95-106 (1965).

126 Hongming Weng, Taisuke Ozaki, and Kiyoyuki Terakura, "Revisiting magnetic coupling in transition-metal-benzene complexes with maximally localized wannier functions," Phys. Rev. B 79, 235118 (2009).

127 R. Yu, X. L. Qi, A. Bernevig, Z. Fang, and X. Dai, "Equivalent expression of Z $Z_{2}$ topological invariant for band insulators using the non-Abelian Berry connection," Phys. Rev. B 84, 75119 (2011).

128 X. J. Wang, J. R. Yates, I. Souza, and D. Vanderbilt, "Ab initio calculation of the anomalous Hall conductivity by Wannier interpolation," Phys. Rev. B 74, 195118 (2006).

129 B. Q. Lv, H. M. Weng, B. B. Fu, X. P. Wang, H. Miao, J. Ma, P. Richard, X. C. Huang, L. X. Zhao, G. F. Chen, Z. Fang, X. Dai, T. Qian, and H. Ding, "Experimental discovery of weyl semimetal taas," Phys. Rev. X 5, 031013 (2015).

130 B. Q. Lv, N. Xu, H. M. Weng, J. Z. Ma, P. Richard, X. C. Huang, L. X. Zhao, G. F. Chen, C. E. Matt, F. Bisti, V. N. Strocov, J. Mesot, Z. Fang, X. Dai, T. Qian, M. Shi, and H. Ding, 
"Observation of Weyl nodes in TaAs," Nature Physics 11, 724-727 (2015).

131 Xiaochun Huang, Lingxiao Zhao, Yujia Long, Peipei Wang, Dong Chen, Zhanhai Yang, Hui Liang, Mianqi Xue, Hongming Weng, Zhong Fang, Xi Dai, and Genfu Chen, "Observation of the chiral-anomaly-induced negative magnetoresistance in 3d weyl semimetal taas," Phys. Rev. X 5, 031023 (2015).

132 B. Q. Lv, S. Muff, T. Qian, Z. D. Song, S. M. Nie, N. Xu, P. Richard, C. E. Matt, N. C. Plumb, L. X. Zhao, G. F. Chen, Z. Fang, X. Dai, J. H. Dil, J. Mesot, M. Shi, H. M. Weng, and H. Ding, "Observation of fermi-arc spin texture in taas," Phys. Rev. Lett. 115, 217601 (2015).

133 Lexian Yang, Zhongkai Liu, Yan Sun, Han Peng, Haifeng Yang, Teng Zhang, Bo Zhou, Yi Zhang, Yanfeng Guo, Marein Rahn, Dharmalingam Prabhakaran, Zahid Hussain, SungKwan Mo, Claudia Felser, Binghai Yan, and Yulin Chen, "Discovery of a Weyl Semimetal in non-Centrosymmetric Compound TaAs," arXiv:1507.00521 (2015).

134 Z K Liu, L X Yang, Y Sun, T Zhang, H Peng, H F Yang, C Chen, Y Zhang, Y F Guo, D Prabhakaran, M Schmidt, Z Hussain, S.-K. Mo, C Felser, B Yan, and Y L Chen, "Evolution of the Fermi surface of Weyl semimetals in the transition metal pnictide family," Nat. Mater. advance online publication (2015), 10.1038/nmat4457.

135 Chandra Shekhar, Ajaya K. Nayak, Yan Sun, Marcus Schmidt, Michael Nicklas, Inge Leermakers, Uli Zeitler, Yurii Skourski, Jochen Wosnitza, Zhongkai Liu, Yulin Chen, Walter Schnelle, Horst Borrmann, Yuri Grin, Claudia Felser, and Binghai Yan, "Extremely large magnetoresistance and ultrahigh mobility in the topological Weyl semimetal candidate NbP," Nature Physics 11, 645-649 (2015).

136 Chenglong Zhang, Cheng Guo, Hong Lu, Xiao Zhang, Zhujun Yuan, Ziquan Lin, Junfeng Wang, and Shuang Jia, "Large magnetoresistance over an extended temperature regime in monophosphides of tantalum and niobium," Phys. Rev. B 92, 041203 (2015).

137 Su-Yang Xu, Ilya Belopolski, Nasser Alidoust, Madhab Neupane, Guang Bian, Chenglong Zhang, Raman Sankar, Guoqing Chang, Zhujun Yuan, Chi-Cheng Lee, Shin-Ming Huang, Hao Zheng, Jie Ma, Daniel S. Sanchez, BaoKai Wang, Arun Bansil, Fangcheng Chou, Pavel P. Shibayev, Hsin Lin, Shuang Jia, and M. Zahid Hasan, "Discovery of a weyl fermion semimetal and topological fermi arcs," Science 349, 613-617 (2015).

138 S.-Y. Xu, I. Belopolski, D. S. Sanchez, C. Zhang, G. Chang, C. Guo, G. Bian, Z. Yuan, H. Lu, 
T.-R. Chang, P. P. Shibayev, M. L. Prokopovych, N. Alidoust, H. Zheng, C.-C. Lee, S.-M. Huang, R. Sankar, F. Chou, C.-H. Hsu, H.-T. Jeng, A. Bansil, T. Neupert, V. N. Strocov, H. Lin, S. Jia, and M. Z. Hasan, "Experimental discovery of a topological Weyl semimetal state in TaP," Science Advances 1, e1501092-e1501092 (2015).

Su-Yang Xu, Nasser Alidoust, Ilya Belopolski, Zhujun Yuan, Guang Bian, Tay-Rong Chang, Hao Zheng, Vladimir N. Strocov, Daniel S. Sanchez, Guoqing Chang, Chenglong Zhang, Daixiang Mou, Yun Wu, Lunan Huang, Chi-Cheng Lee, Shin-Ming Huang, BaoKai Wang, Arun Bansil, Horng-Tay Jeng, Titus Neupert, Adam Kaminski, Hsin Lin, Shuang Jia, and M. Zahid Hasan, "Discovery of a Weyl fermion state with Fermi arcs in niobium arsenide," Nature Physics 11, 748-754 (2015).

Su-Yang Xu, Ilya Belopolski, Daniel S. Sanchez, Madhab Neupane, Guoqing Chang, Koichiro Yaji, Zhujun Yuan, Chenglong Zhang, Kenta Kuroda, Guang Bian, Cheng Guo, Hong Lu, Tay-Rong Chang, Nasser Alidoust, Hao Zheng, Chi-Cheng Lee, Shin-Ming Huang, ChuangHan Hsu, Horng-Tay Jeng, Arun Bansil, Aris Alexandradinata, Titus Neupert, Takeshi Kondo, Fumio Komori, Shik Shin, Hsin Lin, Shuang Jia, and M. Zahid Hasan, "Spin polarization and texture of the Fermi arcs in the Weyl Fermion semimetal TaAs," arXiv:1510.08430 (2015).

141 N. Xu, H. M. Weng, B. Q. Lv, C. Matt, J. Park, F. Bisti, V. N. Strocov, D. gawryluk, E. Pomjakushina, K. Conder, N. C. Plumb, M. Radovic, G. Autès, O. V. Yazyev, Z. Fang, X. Dai, G. Aeppli, T. Qian, J. Mesot, H. Ding, and M. Shi, "Observation of Weyl nodes and Fermi arcs in TaP," arXiv:1507.03983 (2015).

142 D.-F. Xu, Y.-P. Du, Z. Wang, Y.-P. Li, X.-H. Niu, Q. Yao, D. Pavel, Z.-A. Xu, X.-G. Wan, and D.-L. Feng, "Observation of Fermi Arcs in Non-Centrosymmetric Weyl Semi-Metal Candidate NbP," Chinese Physics Letters 32, 107101 (2015).

143 S. M. Huang, S. Y. Xu, I. Belopolski, C. C. Lee, G. Chang, B. K. Wang, N. Alidoust, G. Bian, M. Neupane, C. Zhang, S. Jia, A. Bansil, H. Lin, and M. Z. Hasan, "A Weyl Fermion semimetal with surface Fermi arcs in the transition metal monopnictide TaAs class," Nature Communications 6, $7373(2014)$.

144 Pavan Hosur, Xi Dai, Zhong Fang, and Xiao-Liang Qi, "Time-reversal-invariant topological superconductivity in doped weyl semimetals," Phys. Rev. B 90, 045130 (2014).

145 Shao-Kai Jian, Yi-Fan Jiang, and Hong Yao, "Emergent spacetime supersymmetry in 3d weyl semimetals and 2d dirac semimetals," Phys. Rev. Lett. 114, 237001 (2015). 
146 Yi Li and F. D. M. Haldane, "Topological nodal Cooper pairing in doped Weyl metals," arXiv:1510.01730 (2015).

147 G. Bednik, A. A. Zyuzin, and A. A. Burkov, "Superconductivity in weyl metals," Phys. Rev. B 92, 035153 (2015).

148 H. Wang, H. Wang, H. Liu, H. Lu, W. Yang, S. Jia, X.-J. Liu, X. C. Xie, Wei J., and J. Wang, "Observation of superconductivity induced by a point contact on $3 \mathrm{D}$ Dirac semimetal $\mathrm{Cd}_{3} \mathrm{As}_{2}$ crystals," Nature Materials advance online publication (2015), 10.1038/nmat4456.

149 L. P. He, Y. T. Jia, S. J. Zhang, X. C. Hong, C. Q. Jin, and S. Y. Li, "Pressure-induced superconductivity in the three-dimensional Dirac semimetal $\mathrm{Cd}_{3} \mathrm{As}_{2}, " 1502.02509$ (2015).

150 J. Zhang, F.-L. Liu, J.-K. Dong, Y. Xu, N.-N. Li, W.-G. Yang, and S.-Y. Li, "Structural and Transport Properties of the Weyl Semimetal NbAs at High Pressure," Chinese Physics Letters 32, $97102(2015)$.

151 Hongming Weng, Yunye Liang, Qiunan Xu, Rui Yu, Zhong Fang, Xi Dai, and Yoshiyuki Kawazoe, "Topological node-line semimetal in three-dimensional graphene networks," Phys. Rev. B 92, 045108 (2015).

152 A L Mackay and Terrones H, "Diamond from graphinte," Nature 352, 762 (1991).

153 L. Lu, L. Fu, J. D. Joannopoulos, and M. Soljačić, "Weyl points and line nodes in gyroid photonic crystals," Nature Photonics 7, 294-299 (2013).

154 Makoto Tagami, Yunye Liang, Hisashi Naito, Yoshiyuki Kawazoe, and Motoko Kotani, "Negatively curved cubic carbon crystals with octahedral symmetry," Carbon 76, 266-274 (2014).

155 Shinsei Ryu and Yasuhiro Hatsugai, "Topological Origin of Zero-Energy Edge States in Particle-Hole Symmetric Systems," Physical Review Letters 89, 077002 (2002).

156 T.T. Heikkilä and G.E. Volovik, "Dimensional crossover in topological matter: Evolution of the multiple dirac point in the layered system to the flat band on the surface," JETP Letters 93, 59-65 (2011).

157 T.T. Heikkilä, N.B. Kopnin, and G.E. Volovik, "Flat bands in topological media," JETP Letters 94, 233-239 (2011).

158 G. E. Volovik, "Topology of quantum vacuum," arXiv:1111.4627 (2011).

159 N. B. Kopnin, T. T. Heikkilä, and G. E. Volovik, "High-temperature surface superconductivity in topological flat-band systems," Phys. Rev. B 83, 220503 (2011).

160 G. E. Volovik, "From Standard Model of particle physics to room-temperature superconduc- 
tivity," arXiv:1409.3944 (2014).

161 Y. Chen, Y. Xie, S. A. Yang, H. Pan, F. Zhang, M. L. Cohen, and S. Zhang, "Spin-orbitfree Weyl-loop and Weyl-point semimetals in a stable three-dimensional carbon allotrope," arXiv:1505.02284 (2015).

162 M. Zeng, C. Fang, G. Chang, Y.-A. Chen, T. Hsieh, A. Bansil, H. Lin, and L. Fu, "Topological semimetals and topological insulators in rare earth monopnictides," arXiv:1504.03492 (2015).

163 Youngkuk Kim, Benjamin J. Wieder, C. L. Kane, and Andrew M. Rappe, "Dirac line nodes in inversion-symmetric crystals," Phys. Rev. Lett. 115, 036806 (2015).

164 Rui Yu, Hongming Weng, Zhong Fang, Xi Dai, and Xiao Hu, "Topological Node-Line Semimetal and Dirac Semimetal State in Antiperovskite $\mathrm{Cu}_{3} \mathrm{PdN}, "$ Phys. Rev. Lett. 115, 036807 (2015).

165 Chen Fang, Yige Chen, Hae-Young Kee, and Liang Fu, "Topological nodal line semimetals with and without spin-orbital coupling," Phys. Rev. B 92, 081201 (2015).

166 Lilia S. Xie, Leslie M. Schoop, Elizabeth M. Seibel, Quinn D. Gibson, Weiwei Xie, and Robert J. Cava, "A new form of $\mathrm{Ca}_{3} \mathrm{P}_{2}$ with a ring of Dirac nodes," APL Mater. 3, 083602 (2015), 10.1063/1.4926545.

167 G. Bian, T.-R. Chang, R. Sankar, S.-Y. Xu, H. Zheng, T. Neupert, C.-K. Chiu, S.-M. Huang, G. Chang, I. Belopolski, D. S. Sanchez, M. Neupane, N. Alidoust, C. Liu, B. Wang, C.-C. Lee, H.-T. Jeng, A. Bansil, F. Chou, H. Lin, and M. Zahid Hasan, "Topological Nodal-Line Fermions in the Non-Centrosymmetric Superconductor Compound PbTaSe 2 ," arXiv:1505.03069 (2015).

168 G. Bian, T.-R. Chang, H. Zheng, S. Velury, S.-Y. Xu, T. Neupert, C.-K. Chiu, D. S. Sanchez, I. Belopolski, N. Alidoust, P.-J. Chen, G. Chang, A. Bansil, H.-T. Jeng, H. Lin, and M. Zahid Hasan, "Drumhead Surface States and Topological Nodal-Line Fermions in TlTaSe2," arXiv:1508.07521 (2015).

169 J. Zhao, R. Yu, H. Weng, and Z. Fang, "Topological Node-Line Semimetal in Compressed Black Phosphorus," arXiv:1511.05704 (2015).

170 X. L. Sheng, Z. J. Wang, R. Yu, H. M. Weng, Z. Fang, and X. Dai, "Topological insulator to Dirac semimetal transition driven by sign change of spin-orbit coupling in thallium nitride," Phys. Rev. B 90, 245308 (2014).

171 Timothy H. Hsieh, Junwei Liu, and Liang Fu, "Topological crystalline insulators and dirac 
octets in antiperovskites," Phys. Rev. B 90, 081112 (2014).

172 Toshikaze Kariyado and Masao Ogata, "Low-Energy Effective Hamiltonian and the Surface States of $\mathrm{Ca}_{3} \mathrm{PbO}, " J o u r n a l$ of the Physical Society of Japan 81, 4701 (2012).

173 Tero T Heikkila and Grigori E Volovik, "Flat bands as a route to high-temperature superconductivity in graphite," arXiv:1504.05824 (2015).

174 B. J. Yang and N. Nagaosa, "Classification of stable three-dimensional dirac semimetals with nontrivial topology," Nature Communications 5, 4898 (2014).

175 Yuanping Chen, Yuee Xie, Shengyuan A. Yang, Hui Pan, Fan Zhang, Marvin L. Cohen, and Shengbai Zhang, "Nanostructured carbon allotropes with weyl-like loops and points," Nano Letters 15, 6974-6978 (2015).

176 Ai Yamakage, Youichi Yamakawa, Yukio Tanaka, and Yoshihiko Okamoto, "Line-Node Dirac Semimetal and Topological Insulating Phase in Noncentrosymmetric Pnictides $\operatorname{CaAg} X(X=\mathrm{P}$, As)," Journal of the Physical Society of Japan 85, 013708 (2016).

177 Mazhar N. Ali, Quinn D. Gibson, T. Klimczuk, and R. J. Cava, "Noncentrosymmetric superconductor with a bulk three-dimensional Dirac cone gapped by strong spin-orbit coupling," Phys. Rev. B 89, 020505 (2014). 